
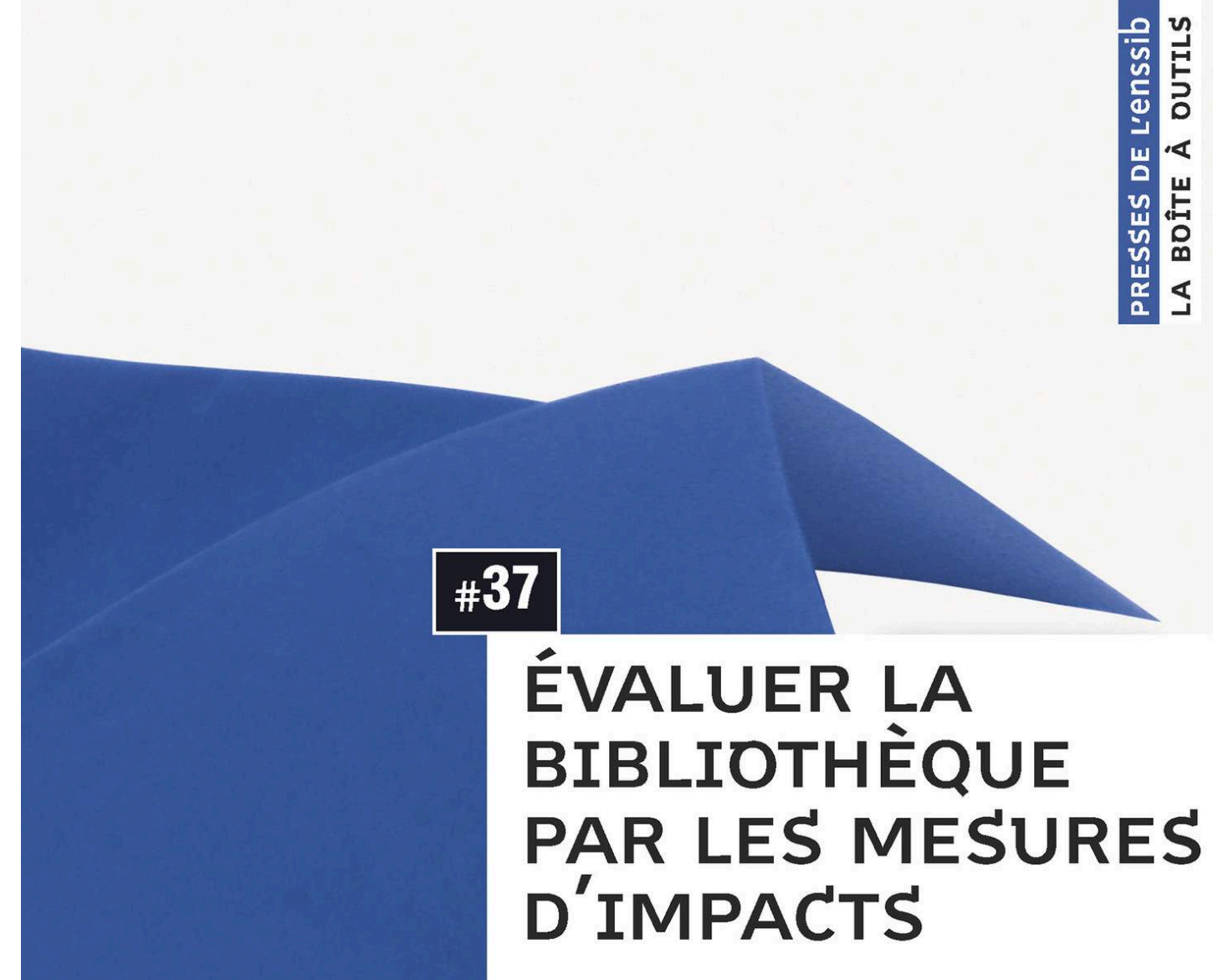

sous la direction de cécile touitou 


\section{Évaluer la bibliothèque par les mesures d'impacts}

\section{Cécile Touitou (dir.)}

DOI : 10.4000/books.pressesenssib.5355

Éditeur: Presses de l'enssib

Lieu d'édition : Villeurbanne

Année d'édition : 2016

Date de mise en ligne : 13 mars 2019

Collection : La Boîte à outils

ISBN électronique : 9782375460917

\section{Sobooks}

http://books.openedition.org

\section{Édition imprimée}

Date de publication : 1 janvier 2016

ISBN : 9791091281768

Nombre de pages : 184

\section{Référence électronique}

TOUITOU, Cécile (dir.). Évaluer la bibliothèque par les mesures d'impacts. Nouvelle édition [en ligne]. Villeurbanne : Presses de l'enssib, 2016 (généré le 01 février 2021). Disponible sur Internet : <http:// books.openedition.org/pressesenssib/5355>. ISBN : 9782375460917 . DOI : https://doi.org/10.4000/ books.pressesenssib.5355.

(C) Presses de l'enssib, 2016

Conditions d'utilisation :

http://www.openedition.org/6540 


\section{\#37}

ÉVALUER LA BIBLIOTHĖQUE PAR LES MESURES D'IMPACTS

sous la direction de cécile touitou 


\section{BAO\#37}

\section{ÉVALUER LA BIBLIOTHĖQUE PAR LES MESURES D'IMPACTS}

Faut-il compter les livres que l'on prête ou bien faut-il interroger les lecteurs sur ce qu'ils ont retenu de leur lecture ? Si la première mesure semble facile à réaliser, comment collecter la seconde? Plus généralement, comment évaluer la performance de sa bibliothèque et comment mesurer son impact sur le public qu'elle dessert ?

Ces questions résument l'évolution récente de l'évaluation, désormais centrée sur la mesure du changement que provoque auprès d'un usager, ou d'un quartier, la présence de la bibliothèque et les services qu'elle offre ou le retour sur investissement - économique et/ou social - pour la collectivité.

Résolument tourné vers " la fonction citoyenne d'inclusion sociale, de lieu de rendez-vous [de la bibliothèque] » (Françoise Benhamou), cet ouvrage fournit un mode opératoire précis pour construire une démarche vertueuse d'évaluation : « un indicateur doit répondre à un objectif et fournir une aide à la prise de décision ».

Cerner l'impact de son établissement, dessiner la cartographie de sa zone de chalandise, mesurer les effets induits par l'offre de services - présentiels ou virtuels - sur ses différents publics, calculer la satisfaction et tester, à toutes les étapes d'un projet, l'expérience de l'usager sont les sujets traités ici.

Coordonné par Cécile Touitou, praticienne de la question, et actuellement responsable marketing à la bibliothèque de Sciences Po Paris, ce volume collectif réunit des professionnel.le.s, tous et toutes impliqué.e.s dans le pilotage et l'accompagnement des actions d'évaluation - de la lecture publique comme de l'enseignement supérieur et de la recherche.

Mots clés : évaluations - expériences utilisateurs - impact économique et sociétal - normes - ressources numériques - tests utilisateurs - zone de chalandise 


\section{\#37}

ÉVALUER

LA BIBLIOTHĖQUE

PAR LES MESURES

D'IMPACTS

Sous la direction de cécile rouitou 
Ont contribué à cet ouvrage:

Nicolas Alarcon

Muriel Amar

Emmanuelle Asselin

Cécile Avallone

Françoise Benhamou

Frédéric Brodkom

Nathalie Clot

Hélène Coste

Nadine Delcarmine
Frank Huysmans

Marie-Christine Jacquinet

Thomas Jouneau

Marc Maisonneuve

Marjolein Oomes

Joris Paillaré

Thomas Porquet

Guilaine Thuillart

Cécile Touitou

Évaluer la bibliothèque par les mesures d'impacts / sous la dir. de Cécile Touitou. Villeurbanne : Presses de l'enssib, cop. 2016. - 1 vol. (184 p.) ; $21 \mathrm{~cm}$. - (La Boîte à outils ; 37 ; ISSN 1259-4857)

ISBN 979-10-91281-76-8. (br.) : $22 €$

Autre édition sur un support différent :

Évaluer la bibliothèque par les mesures d'impacts / sous la dir. de Cécile Touitou. Villeurbanne : Presses de l'enssib, cop. 2016. (La Boîte à outils ; 37 ; ISSN 2492-7589)

ISBN 979-10-91281-77-5 : 13,20€

Dewey :

027

Rameau :

Bibliothèques -- Publics -- Attitudes

Bibliothèques -- Services aux publics

Bibliothèques -- Aspect économique

Bibliothèques -- Aspect social

Bibliothèques - Évaluation

Conception centrée sur l'utilisateur

Notice rédigée par la bibliothèque de l’Enssib. 


\section{SOMMAIRE}

MODE D'EMPLOI.

par Cécile Touitou

\section{PARTIE I. CONTEXTE}

$++++++++++++++++++++++++++++++++$

MESURES EN BIBLIOTHĖQUE :

PANORAMA ET ÉVOLUTION

par Nadine Delcarmine. .16

LA MESURE EN BIBLIOTHĖQUE :

UN USAGE INSCRIT DANS LEUR

HISTOIRE

LA NORMALISATION POUR LES

BIBLIOTHĖQUES : 1974-2016 . .21

LIBRES RÉFLEXIONS AU SUJET DES MÉTHODES ET DES ENJEUX DE L'IMPACT ÉCONOMIQUE ET SOCIÉTAL

DES BIBLIOTHĖQUES

par Françoise Benhamou

DES ACTIVITÉS

ÉCONOMIQUES DIVERSIFIÉES

LES DEUX APPROCHES

COMPLÉMENTAIRES

ÉTABLIR LE RETOUR SUR

INVESTISSEMENT :

DEUX CAS CONCRETS

UNE ANALYSE CRITIQUE ET

QUELQUES PERSPECTIVES : L'EXEMPLE

DES BU DU ROYAUME-UNI. .36
PARTIE II. MÉTHODES ET OUTILS

$++++++++++++++++++++++++++++++++$

CONSTRUIRE UN TABLEAU DE BORD POUR LA BIBLIOTHĖQUE?

par Marc Maisonneuve .40

DE L'ESGBU ET DU RAPPORT SLL

AU TABLEAU DE BORD URBAIN 40

LA CONTRIBUTION DES BIBLIOTHĖQUES

À UNE MEILLEURE GOUVERNANCE ....... 42

LE PÉRIMÈTRE DU TABLEAU

DE BORD URBAIN

\section{ÉVALUER L'IMPACT DE}

SA BIBLIOTHĖQUE SUR LE TERRITOIRE : MESURER LA ZONE DE CHALANDISE OU DE RAYONNEMENT DE SA BIBLIOTHĖQUE

par Emmanuelle Asselin .52

QU'EST-CE QU'UNE ZONE DE CHALANDISE OU DE RAYONNEMENT?

QUELS INDICATEURS RETENIR POUR ÉVALUER LA ZONE DE CHALANDISE DE SA BIBLIOTHĖQUE?

MESURER LA VALEUR SOCIÉTALE

DE LA BIBLIOTHĖQUE PUBLIQUE :

DÉFINITIONS, CRITÈRES ET MÉTHODES

par Frank Huysmans

et Marjolein Oomes. .65

DES INTRANTS ET EXTRANTS AUX OUTCOMES 
IMPACT, VALEUR, OUTCOME : EFFORT

DE CLARIFICATION TERMINOLOGIQUE..68

MESURER LA VALEUR SOCIÉTALE

DES BIBLIOTHĖQUES PUBLIQUES

ARRÊTER, COMMENCER, CONTINUER :

ÉVALUER POUR DÉCIDER

par Nathalie Clot .75

SAVOIR POUR DÉCIDER :

MAÎTRISE OU ILLUSION ? 76

LESS IS MORE : QUAND SE LIBÉRER DE L'INDICATEUR TYPE PERMET

DE CHANGER LE SERVICE 81

PLAIDOYER POUR UNE DÉMARCHE DE DESIGN ORIENTÉ UTILISATEUR (DESIGN UX)

\section{ÉVALUER ET INSPIRER}

LES TERRITOIRES DE DEMAIN

par Marie-Christine Jacquinet

DU DIAGNOSTIC DE LA LECTURE

À UNE LOGIQUE DE TERRITOIRE

LE PILOTAGE AU CCEUR

D'INTERACTIONS PROSPECTIVES

.92

MESURER LA FRÉQUENTATION DE 121

ÉTABLISSEMENTS : L'EXEMPLE DE LA

BIBLIOTHĖQUE DÉPARTEMENTALE DU

VAL D'OISE

par Cécile Avallone 96

COMPTER QUOI ET COMMENT ? . .96

QUELS RÉSULTATS POUR LE VAL D'OISE?

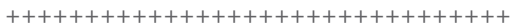
ENCADRÉS :

Six types d'outcomes pour les bibliothèques publiques ............ 68

Trois fiches diagnostic

Mesurer la fréquentation :

les cinq étapes de la mise en œuvre...

$++++++++++++++++++++++++++++++++$

PARTIE III. PILOTAGE

$++++++++++++++++++++++++++++++++$

ÉVALUER LES FORMATIONS

À LA MAÎTRISE DE L'INFORMATION

À L'UNIVERSITÉ : CONDITIONS, ENJEUX ET PERSPECTIVES

par Hélène Coste 100

LES TROIS ÉTAPES

DE L'ÉVALUATION. 100

LE SERVICE PUBLIC POSTÉ EN

BIBLIOTHĖQUE : COMMENT

MESURER L'EFFICACITÉ D'UN

SERVICE ORDINAIRE ET DISCRET ?

L'EXPÉRIMENTATION DE LA MÉTHODE AMÉRICAINE READ À LA BPI

par Muriel Amar

et Guilaine Thuillart.

PAROLES DES USAGERS, PAROLES DES

BIBLIOTHÉCAIRES : QUE CHOISIR ?..109

MISE EN GEUVRE ET DISCUSSION.........117

ÉVALUER LES ATTENTES ET

PERCEPTIONS : L'OUTIL LIBQUAL+@, SES AVANTAges et SES LIMITES

par Frédéric Brodkom 119

L'ENQUÊTE LIBQUAL 
MESURER LES EFFETS

DE SON ACTION. 120

L'INTÉRÊT D'UTILISER LIBQUAL 120

LES LIMITES À L'USAGE

DE LIBQUAL

ÉVALUER LA CONSULTATION SUR PLACE ET L'USAGE DES ESPACES : LE CAS DE LA BIBLIOTHĖQUE DE SCIENCES PO PARIS

par Cécile Touitou 128

PASSER DE LA MESURE DES

COLLECTIONS

AUX USAGES DES PUBLICS 128

QUI SONT LES USAGERS ET QUE FONT-

ILS ? : DES ENQUÊTES 130

MAIS S'ILS LISENT, CONSULTENT,

FEUILLĖTENT, QUE REGARDENT-ILS

EXACTEMENT ?

LE TRI PAR CARTES POUR AMÉLIORER L'ARBORESCENCE DU SITE WEB DE LA BIBLIOTHĖQUE DE SCIENCES PO PARIS

par Joris Paillaré 137

ASSIGNER UN OBJECTIF

À CHAQUE TRI

PRÉPARATION.

ANALYSE ET RÉSULTATS

\section{ÉVALUER L'USAGE}

DES RESSOURCES NUMÉRIQUES : QUELS ENJEUX, QUELLES SOLUTIONS ?

par Thomas Jouneau et Thomas

Porquet .140

POURQUOI ET COMMENT TRAVAILLER

AVEC EZPAARSE?
L'EXPLOITATION DU LOGICIEL : LE CAS

DE L'UL 145

PERSPECTIVES 148

ÉVALUER L'UTILISABILITÉ DES SITES WEB

par Nicolas Alarcon 150

LES TESTS D'UTILISABILITÉ 150

CRÉER UN TEST D'UTILISABILITÉ ....... 152

NI PANACÉE, NI CONCLUSION .157

$++++++++++++++++++++++++++++++++$ ENCADRÉS :

Recommandations

de la norme ISO 16439

Les trois temps du tri par cartes.

Présentation d'une ligne

de log standard

Améliorer l'utilisabilité du site : quatre bénéfices principaux

$++++++++++++++++++++++++++++++++$

MÉMENTO

par Cécile Touitou.

GLOSSAIRE

INDEX.

BIBLIOGRAPHIE .175

LISTE DES ILLUSTRATIONS 179

LISTE DES AUTEURS 181 
Au moment de la composition du sommaire de cette Boîte à Outils, nous apprenions le décès de Bertrand Calenge.

Imaginer réfléchir collectivement sur l'évaluation en bibliothèque sans son éclairage était une tâche triste et difficile.

En 2008, il terminait un article ${ }^{*}$ dans le Bulletin des bibliothèques de France par ces mots : "Ou alors on décide que la bibliothèque appartient à la complexité du vivre ensemble, participe de la grande interrogation des hommes sur leur existence, leur avenir et leur "savoir ensemble", et ses actions relèvent de l' "art de faire", de la politique au sens noble en somme: dans ce cas, il faut bien sûr veiller $\grave{a}$ la bonne utilisation des deniers publics et au suivi de projets particuliers, avec les tableaux de bord adéquats à chaque activité ou projet, et cesser de rêver à l'utopie d'un grand tableau de bord de la bibliothèque ».

Nous avons essayé de suivre ses conseils en faisant un pas de côté, loin d'une pensée unique et normative en essayant de penser l'évaluation dans le concret, le précis, le complexe d'une bibliothèque qui "participe de la grande interrogation des hommes sur leur existence, leur avenir et leur "savoir ensemble" ». Rien moins que ça!

* Bertrand Calenge, "Quels tableaux de bord ? ", Bulletin des bibliothèques de France, 2008, $\mathrm{n}^{\circ}$ 3, pp. 35-38. [En ligne] : < http://bbf.enssib.fr/consulter/bbf-2008-03-0035-004 >. 


\section{MODE D'EMPLOI par Cécile Touitou}

\section{ÉVOLUTION DES POLITIQUES D'ÉVALUATION}

Il n'y a pas si longtemps, les bibliothèques, à l'image d'une société relativement cloisonnée, vivaient en vase clos, ignorant les spécificités du public qu'elles desservaient, faisant la sourde oreille à leurs attentes, méconnaissant le territoire où elles étaient implantées, indifférentes aux acteurs associatifs qui œuvraient à quelques rues d'elles ; n'entretenant finalement qu'une relation verticale avec l'échelon administratif supérieur $^{1}$ dont elles dépendaient : la collectivité territoriale, l'université ; ou avec leur ministère de rattachement (Culture ou Enseignement supérieur). C'était l'époque de la politique de l'offre, où la demande, dans les établissements culturels, était considérée - au mieux - avec circonspection.

\section{AVANT 1990 : L'ÉVALUATION BIBLIO-CENTRÉE}

De cette époque (re)constituée en repoussoir, l'évaluation retiendra les rapports statistiques demandés chaque année par les administrations centrales aux établissements de lecture publique et aux bibliothèques universitaires sur la base d'indicateurs ${ }^{* 2}$ normalisés, sans reflet ni des missions propres des établissements, ni des besoins spécifiques de ses usagers. Ces documents perdurent. Ils interrogent les bibliothèques sur leur activité : la composition du personnel ; les budgets ; les volumes de prêts, de collection, des ressources électroniques, de places et de chaises ; ainsi que le nombre des inscrits actifs. Ils ont le mérite de soumettre aux mêmes indicateurs l'ensemble des établissements qui peuvent ainsi se comparer aux établissements identiques et disposer de séries chronologiques.

1. Lire sur le sujet, Dominique Lahary, « À bas les tutelles ! », DLog, 8 juin 2011. [En ligne] : < https://lahary.wordpress.com/2011/06/08/a-bas-les-tutelles/ >.

2. Les termes suivis d'un astérisque (à leur première occurrence) sont définis dans le glossaire en fin d'ouvrage. 


\section{LES ANNÉES 2000 : LE PUBLIC S'IMPOSE}

Les bibliothèques ont ensuite été ébranlées par des évolutions majeures qui depuis une vingtaine d'années secouent les sociétés occidentales... Arrivée de l'Internet qui met l'information à disposition de tous (ou presque), bouleversement du statut de l'auteur, prégnance grandissante des communautés qui commentent, promeuvent et partagent. Pour les bibliothèques, c'est l'évolution des outils qui devait permettre aux usagers d'entrer au cœur du dispositif. Ainsi, dans le milieu des années 2000, les catalogues ont ouvert leurs notices aux commentaires ; on a rêvé un moment que les lecteurs proposeraient des tags développant une indexation en langage naturel générant des nuages de mots qui auraient favorisé une navigation intuitive ${ }^{3}$.

\section{LES ANNÉES 2010 : LES BIBLIOTHĖQUES DANS UN MONDE CONCURRENTIEL}

Au cœur de cette évolution, la montée en puissance de l'information dématérialisée disponible immédiatement sur le Web, la portabilité des outils de consultation, l'évolution rapide des pratiques ont fait basculer les bibliothèques dans un monde concurrentiel, allant jusqu'à mettre en péril leur existence. Pour certains financeurs, dans un contexte de réduction massive des budgets, qu'est-ce qui justifie encore de dépenser pour des établissements de lecture publique ? Pourquoi ne remplacerait-on pas les bibliothèques universitaires par des salles de travail équipées d'ordinateurs et de wi-fi, de tables et de chaises ? Plus besoin de documentation puisque « tout est sur Internet ! » Plus besoin de livres puisqu' « ils ne lisent plus ! ». On le voit, les bibliothèques sont contestées dans leur existence-même ${ }^{4}$.

Ces considérations ne sont pas si éloignées des questions d'évaluation auxquelles est consacré ce volume de la collection la Boîte à outils. Parallèlement à ces mutations sociétales, dans un contexte de réduction des

3. Howard Strauss, "Library portals: A minority report”, NetConnect, 2002, 127(17), pp. 34-37. Sur la possibilité de tagguer les notices, se souvenir de l'outil Aquabrower de la société néerlandaise Medialab qui a été proposé dans de nombreux OPAC.

4. " Près de 350 bibliothèques ont fermé en Grande-Bretagne au cours des six dernières années, provoquant la perte de près de 8000 emplois ». Voir "Libraries facing 'greatest crisis' in their history", The Guardian, 29 mars 2016. [En ligne] : < http://www.theguardian.com/books/2016/ mar/29/libraries-facing-greatest-crisis-in-their-history >. 
budgets publics, on a assisté à l'avènement des politiques d'évaluation. En France, la loi organique relative aux lois de finances (LOLF) promulguée en 2001 s'applique à toute l'administration depuis 2006. Elle impose aux gestionnaires de rendre des comptes sur l'efficacité de l'utilisation des crédits qui leur sont attribués. Pour rendre compte, il convient de mesurer. Cette pratique - devenue bientôt culture ? - oblige à réfléchir à son rôle et à ses missions.

Fini le temps (s'il a jamais existé) où la bibliothèque fonctionnait hors sol en se demandant combien d'ouvrages avaient quitté les rayonnages (pour être lus ? Personne ne pouvait le garantir). Aujourd'hui, certains prétendent qu'il convient de demander à l'usager quel profit il a tiré de cet emprunt... Cette lecture l'a-t-elle transformé ?

\section{LA MESURE D'IMPACT ET LA SATISFACTION DES USAGERS}

Effectivement, bousculés par les mutations qui se sont produites dans un paysage de plus en plus concurrentiel, il est devenu difficile pour les personnels des bibliothèques de se contenter d'évaluer l'activité des services sans vérifier la satisfaction* qu'en tirent les usagers et en mesurer l'impact auprès d'eux. La bibliothèque, comme l'ensemble des services marchands et publics, a peu à peu mis le public et l'usager au cœur de son activité en tentant de déployer une offre de services adaptée à ses besoins qui vise à combler sa satisfaction.

\section{ORGANISATION DU LIVRE}

\section{MÉTHODOLOGIE DE L'ÉVALUATION DE L'IMPACT}

Cette démarche vertueuse trouve son écho dans l'évolution des normes successives présentées en tête de cet ouvrage par Nadine Delcarmine. Les recommandations de l'Afnor en matière d'évaluation ont d'abord traité de la mesure de l'activité, de sa performance pour en venir tout récemment (2014) à son impact.

La grande nouveauté de la norme ISO 16439 est de souligner l'importance d'une évaluation en relation avec les missions et les besoins des usagers. Françoise Benhamou revient sur « l'appétit soudain pour l'économie qui 
traverse les mondes de la culture ». Après avoir détaillé les principes des études d'impact économique et l'évaluation du retour sur investissement au travers de quelques exemples étrangers récents, elle s'interroge sur leur validité et met en garde contre " la propension à l'économisme". Marc Maisonneuve développe les outils pour construire ce tableau de bord urbain où les indicateurs de la bibliothèque reflètent ses missions en lien avec le droit à la culture, le droit à l'information et le droit à la formation, et sont pensés en fonction des objectifs globaux du territoire à desservir. Emmanuelle Asselin explique la notion de territoire qui est le terreau nourricier dans lequel la bibliothèque va puiser sa raison d'être. Elle nous dit comment évaluer la zone de chalandise* ou de rayonnement de l'établissement en tirant parti de toutes les informations que met l'Insee à disposition de tous.

\section{MISE EN GEUVRE D'UNE DÉMARCHE D'ÉVALUATION ORIENTÉE USAGER}

Frank Huysmans et Marjolein Oomes évoquent en détail la notion d'outcome* qui précise celle de l'impact en la reliant aux missions propres à chaque bibliothèque. Des études menées aux Pays-Bas leur ont permis de définir une véritable typologie des impacts. Les missions n'étant pas les mêmes d'un établissement à l'autre, il convient de retenir des indicateurs qui seront des outils d'aide à la décision dans une appréhension territoriale de l'offre de services.

Nathalie Clot expose comment la démarche utilisateur* (UX : user experience) permet de donner un sens à son activité et de " concevoir une bibliothèque attentive à ses utilisateurs ». La réflexion sur les missions, l'expression des besoins par les usagers, l'observation des usages, la mesure de la satisfaction : quatre étapes de la réflexion et de l'analyse qui viendront ainsi nourrir une démarche d'évaluation qui mène à l'action et permet des boucles de rétro-action vertueuses pour un véritable pilotage par objectifs qui se nourrit de l'expérience usager.

Marie-Christine Jacquinet montre comment le département des Yvelines pense « la performance de la lecture publique [...] adossée à une démarche de développement territorial ». La bibliothèque s'insère dans le tissu des acteurs locaux et co-participe à une offre de services résolument tournée vers les besoins des usagers. 


\section{DES OUTILS POUR L'ÉVALUATION DES USAGES}

La troisième partie de l'ouvrage dépeint des cas concrets qui illustrent la nécessité de penser la bibliothèque comme outil de transformation des usagers : accès à la connaissance, accès aux ressources imprimées ou à distance, formation tout au long de la vie, formation aux compétences informationnelles... Proposer une offre de services en adéquation avec les besoins des utilisateurs, c'est ce que met en œuvre la méthodologie Libqual+ présentée par notre collègue de l'université de Louvain, Frédéric Brodkom. Cette enquête internationale mesure les attentes, le désiré et le perçu d'une trentaine de services de la bibliothèque ; elle permet la comparaison dans le temps, et avec les autres établissements. Puis Hélène Coste explicite les expériences d'évaluation de la formation en milieu universitaire et combien il serait souhaitable de pouvoir mesurer le bénéfice que tirent les étudiants des formations qu'ils reçoivent. Muriel Amar et Guilaine Thuillart mettent en avant une étude d'évaluation des interactions en service public, à la Bibliothèque publique d'information (Bpi), permettant au bibliothécaire de coder l'effort et l'expertise mobilisés pour répondre aux usagers. Cécile Touitou montre comment la bibliothèque de Sciences Po Paris déploie une gamme complète d'études permettant de cerner les usages des espaces et de la documentation. Thomas Jouneau et Thomas Porquet présentent la question stratégique de la mesure des accès aux ressources numériques, longtemps restés une boîte noire, mais qui peuvent désormais être analysés finement en segmentant publics et usages. Enfin, Nicolas Alarcon clôture cette partie en présentant l'importance de l'évaluation de l'utilisabilité des sites web, illustrant parfaitement le propos qui sous-tend l'ensemble de l'ouvrage : proposer des services qui servent à l'usager et dont l'usage est conçu pour lui et validé par lui-même !

\section{UN PROCESSUS D'AMÉLIORATION CONTINUE}

À leur façon, chacune de ces contributions illustre une recommandation intéressante faite dans la norme sur les mesures d'impacts (ISO 16439) incitant les bibliothécaires à croiser les méthodologies d'enquête et à combiner les données induites* des chiffres collectés automatiquement (les entrées, les prêts, les consultations des ressources électroniques), avec des données sollicitées* (sur la satisfaction, par exemple), et des données 
observées*. Ce faisceau de mesures permet d'appréhender l'impact sociétal des bibliothèques qui, pour reprendre le titre de la dernière campagne de l'American Library Association ${ }^{5}$ (ALA), « transforment la vie » des citoyens et des quartiers - qu'ils en soient, ou non, usagers directs - où elles sont implantées.

Cette Boîte à outils contribuera, je l'espère, à fournir des pistes concrètes pour permettre à chaque établissement, à la mesure de ses moyens, de mieux connaître ses publics et de cerner ses besoins ; d'évaluer la présence de la bibliothèque et son action sur son territoire de rayonnement ; de déployer une offre de services en adéquation avec les attentes et nourrie de l'observation des usages. Aller au bout de la démarche consistera à interroger l'usager, sa satisfaction, mais aussi les gains en matière de compétences informationnelles, ou la réussite dans ses études ou sa recherche d'emploi. Toute la difficulté sera d'isoler l'impact de la bibliothèque* parmi un nombre important de facteurs. Il ne faudra pas ignorer cette difficulté. Cependant, s'attacher à mener dans la durée une démarche vertueuse d'évaluation partant de l'expression des besoins et de l'observation des usages en amont, et intégrant la mesure de l'activité et celle de l'impact, permettra de dégager de puissants outils d'aide à la décision. Car, ces mesures n'auront de sens que si, finalement, elles permettent de passer à l'action !

5. Libraries Transform: An initiative of the American Library Association. [En ligne] : < http://www. ilovelibraries.org/librariestransform/ >. 


\section{PARTIE I}

\section{CONTEXTE}

1. MESURES EN BIBLIOTHĖQUE : PANORAMA ET ÉVOLUTION par Nadine Delcarmine

$++++++++++++++++++++++++++++++++++++++++++++++++++++++++++++++++$

2. LIBRES RÉFLEXIONS AU SUJET DES MÉTHODES ET DES ENJEUX DE L'IMPACT ÉCONOMIQUE ET SOCIÉTAL DES BIBLIOTHĖQUES par Françoise Benhamou 


\begin{tabular}{|l|l|}
\hline \multicolumn{1}{l|}{} & 1 \\
\hline MESURES EN BIBLIOTHĖQUE : & par \\
PANORAMA ET ÉVOLUTION & Nadine \\
\hline
\end{tabular}

«Ce qui compte ne peut pas toujours être compté, et ce qui peut être compté ne compte pas forcément. »1

Albert Einstein

\section{LA MESURE EN BIBLIOTHĖQUE : UN USAGE INSCRIT DANS LEUR HISTOIRE}

En 2011, Pierre Carbone a décrit les évolutions de la mesure des bibliothèques et de leur évaluation dans sa contribution à l'ouvrage Évaluer la bibliothèque $e^{2}$ intitulée "Évaluation et statistiques dans l'administration des bibliothèques françaises ».

De l'obligation faite aux bibliothèques dès le $\mathrm{XIX}^{\mathrm{e}}$ siècle de produire un rapport annuel, en passant par la création de l'enquête statistique par la Direction des bibliothèques et de la lecture publique au lendemain de la seconde guerre mondiale, jusqu'à la collecte et la publication actuelles des données des bibliothèques publiques par l'Observatoire de la lecture publique $^{3}$ placé sous l'autorité du Service du livre et de la lecture (SLL) et, depuis 2014, à la version révisée de l'Enquête statistique générale auprès des services documentaires de l'enseignement supérieur (ESGBU), les données statistiques des bibliothèques s'inscrivent dans une histoire longue qui traduit le souci de l'État français de connaître et impulser l'activité des bibliothèques et de suivre l'utilisation des deniers publics qui leur sont affectés.

1. Cité dans Marc Romainville, Rémi Goasdoué et Marc Vantourout (dir.), Évaluation et enseignement supérieur : [issu du $23^{e}$ colloque international sur le thème évaluation et enseignement supérieur, qui s'est tenu à l'université Paris Descartes en janvier 2011, organisé par l'Association pour le développement des méthodologies d'évaluation en éducation], Bruxelles, De Boeck, 2013 (coll. Pédagogies en développement).

2. Valérie Alonzo, Pierre-Yves Renard (dir.), Évaluer la bibliothèque, Paris, Éditions du Cercle de la Librairie, 2012 (coll. Bibliothèques), pp. 29-43.

3. Voir dans le Mémento, partie Se comparer aux autres : de l'usage du benchmark, p. 162. 


\section{DES ENJEUX ET DES PRATIQUES PROFONDÉMENT RENOUVELÉS}

La mesure de l'activité des bibliothèques a été affectée par deux puissants mouvements de fond ces dernières années :

- dès le milieu de la décennie 1990, les bibliothèques, rompant avec leur orientation traditionnelle axée sur la constitution de collections imprimées et leur conservation, ont entamé un lent processus de recentrage de leur activité sur les services au public. Portés par les missions dévolues aux bibliothèques (lieux de construction et de diffusion des savoirs et de la culture, outils pour l'égalité des chances et le partage de la connaissance), les bibliothécaires se sont préoccupés d'organiser la rencontre des ressources documentaires détenues ou mises en accès par la bibliothèque avec ses usagers et de répondre à leurs attentes ;

- au tournant du III ${ }^{\mathrm{e}}$ millénaire, les collections numériques sont apparues et se développent depuis à un rythme exponentiel dans les sciences exactes bien sûr mais pas uniquement. Avec Internet, la révolution du support et de l'accès à l'information engendre une révolution des usages de la documentation sous toutes ses formes, les conditions de l'accès au savoir et à la culture sont bouleversées. Le développement du livre numérique est un enjeu majeur des prochaines années pour le grand public comme pour la formation tandis que pour les chercheurs l'urgence réside dans la fouille de données et de textes à partir de ressources en libre accès ou payantes.

C'est ainsi que de données brutes exploitées essentiellement à l'échelle nationale, on est passé à des indicateurs sophistiqués utiles en local, à l'échelon national et/ou international.

En lien avec ces transformations, les bibliothécaires ont renouvelé et développé les services offerts aux publics de tous types, ils ont déployé nombre de compétences nouvelles pour accompagner ces transformations et pratiquent une veille active. 


\section{par les évolutions de la documentation et des pratiques documentaires}

Il s'agit maintenant de redonner de la visibilité à leur action, mais le recueil de données quantitatives fiables (mais aussi qualitatives) sur les services se fait dorénavant dans une complexité croissante. Avec le numérique, les bibliothèques sont passées d'une logique de stock à une logique de flux. Mesurer les usages de collections numériques dont les bibliothèques ne sont pas propriétaires mais qu'elles paient à prix d'or reste aujourd'hui encore délicat, en dépit des avancées obtenues depuis l'apparition de la norme de comptage Counter* et d'outils de suivi des usages tel ezPAARSE ${ }^{4}$. Il est, par exemple, plus difficile d'appréhender des flux documentaires que des emprunts de collections physiques ; la multiplicité et la variété des usages sur supports numériques décuplent les démarches de comptage. Au final, pour percevoir la globalité de l'action des bibliothèques, il n'est pas encore si naturel d'additionner des entrées physiques dans les bibliothèques et des connexions à des serveurs, ni des prêts de documents physiques et des visualisations/déchargements de documents ou parties de documents.

\section{Par les évolutions des environnements institutionnels}

Depuis les années 1980 et plus encore le début des années 2000, cette évaluation des bibliothèques a évolué dans des environnements institutionnels profondément renouvelés avec un État de moins en moins directement gestionnaire des bibliothèques suite aux lois de décentralisation qui ont d'abord affecté les relations entre l'État et les collectivités territoriales avant de modifier les liens entre l'État et les universités au travers de la loi relative aux libertés et responsabilité des universités (LRU) et de l'application des responsabilités et compétences élargies (RCE) de ces mêmes universités.

Pierre Carbone souligne que l'État est dès lors « conduit à piloter une démarche contractuelle avec les collectivités territoriales et les établissements publics. Le contrat constitue l'accord sur les objectifs partagés

4. Counter et ezPAARSE sont présentés dans la contribution de Thomas Jouneau et Thomas Porquet : «Évaluer l'usage des versions numériques : quels enjeux, quelles solutions ? », p. 140. 
par l'État et la collectivité, le point de rencontre entre la démarche locale de projet et les orientations nationales. [...] La collectivité ou l'établissement définit ses priorités et ses objectifs en fonction d'une démarche stratégique globale, ce qui amène la bibliothèque à mettre l'accent sur le soutien et l'accompagnement de ces axes stratégiques. [...] De ce fait, la définition des objectifs et des indicateurs n'est pas réservée au spécialiste du secteur, mais résulte idéalement d'un dialogue entre les décideurs, les usagers et les gestionnaires. Autrement dit, l'évaluation des bibliothèques n'est plus la seule affaire des bibliothécaires ou de leurs gestionnaires directs à l'administration centrale, mais devient l'affaire de tous. $»^{5}$ La LOLF, depuis 2001, fixe un nouveau cadre à la dépense publique en caractérisant l'État au travers de trente-trois missions, Ratios, pourcentages, taux, délais, indicateurs qualitatifs tels les taux de satisfaction, tableaux de bord ont fait leur apparition comme outils, instruments de l'évaluation. Pour Pierre Carbone, « les résultats des bibliothèques sont certes évalués en eux-mêmes, mais aussi de plus en plus en relation avec des politiques globales à l'échelle de la collectivité ou de l'établissement, ou plus généralement de la société. ${ }^{6}$ Les décideurs s'intéressent à la performance, les usagers à la façon dont leurs besoins et attentes sont couverts ainsi qu'à la qualité des services rendus. Quant aux interactions entre la bibliothèque et son environnement, elles sont mesurées au travers de l'impact des bibliothèques (culturel, social, réussite des étudiants, progrès de la recherche). Enfin, les contraintes économiques conduisent à mettre les coûts de la bibliothèque en rapport avec sa valeur ajoutée.

Un débat est ouvert par ces évolutions ; il consiste notamment à déterminer si la quantification de l'action publique n'est pas désormais opérée à l'aune du résultat et de la performance attendue plutôt que comme production de savoir pour éclairer la décision. Ainsi, Florence Jany-Catrice écrit-elle : « au lieu d'opérer comme des points de repère parmi d'autres, importants et utiles à des formes de connaissance, les indicateurs de performance

5. Pierre Carbone, "Évaluation et statistiques dans l'administration des bibliothèques françaises », in Valérie Alonzo, Pierre-Yves Renard (dir.), Évaluer la bibliothèque, Paris, Éditions du Cercle de la Librairie, 2012 (coll. Bibliothèques), pp. 29-43, ici p. 34.

6. Pierre Carbone, "Évaluation et statistiques dans l'administration des bibliothèques françaises », op. cit., p. 43. 
apparaissent rapidement comme une convention validée, comme si, dès lors qu'on énonce un chiffre pour quantifier une réalité sociale, celle-ci renvoyait spontanément à un savoir accepté et explicitement exprimé. Autrement dit l'espace de controverse qui pourrait animer l'annonce de données chiffrées n’apparaît pas ou plus comme une évidence. »7

\section{Albert Ogien relève que :}

« des formes traditionnelles de dénombrement permettant de fournir une connaissance de l'état de la société et de ses ressortissants pour gouverner de façon appropriée (une statistique descriptive et explicative que l'Insee ou d'autres organismes continuent de produire), on est passé à une statistique prescriptive dans laquelle le chiffre cesse d'être un instrument de savoir, de débats et de préparation à la décision pour devenir la source même des règles qui déterminent, de façon plus ou moins automatisée, l'orientation et le contenu des politiques publiques.

La caractéristique première du système du chiffre gestionnaire est que la quantification qu'il opère vise à soumettre l'action publique au principe d'efficacité [...].

[...] l'idée s'impose qu'un gouvernement est efficace lorsqu'il parvient à rendre la moindre dépense qu'il engage exactement ajustée au résultat qu'elle produit au meilleur coût ;

[...] l'efficacité est intégralement définie à partir de la notion économique d'optimalité (ou d'efficience). Or si le souci d'employer les deniers publics de façon économe et utile est difficilement contestable, la question se pose tout de même de savoir si l'optimalité est un principe qu'on peut appliquer au politique. ${ }^{8}$

7. Florence Jany-Catrice, La performance totale : nouvel esprit du capitalisme ?, Villeneuve d'Ascq, Presses universitaires du Septentrion, 2012 (coll. Capitalismes-éthique-institutions ; 3), p. 25.

8. Albert Ogien, Désacraliser le chiffre dans l'évaluation du secteur public : conférences-débats organisées par le groupe Sciences en questions à l'Inra en 2012 le 24 janvier à Rennes et le 14 février à Paris, Versailles, Éd. Quae, 2013, pp. 49-50. 


\section{LA NORMALISATION POUR LES BIBLIOTHĖQUES : 1974-2016}

\section{UNE COMMISSION DE L'ASSOCIATION FRANÇAISE DE NORMALISATION (AFNOR), MIROIR DU COMITÉ TECHNIQUE ISO (ORGANISATION INTERNATIONALE DE NORMALISATION)}

C'est la Commission nationale 46-8 de l'Afnor (Qualité - statistiques et évaluation des résultats) qui a pour mission d'élaborer et suivre les normes relatives à l'évaluation des résultats des bibliothèques (pris dans un sens générique), musées et archives. Son activité, soutenue par le ministère de la Culture et de la Communication ainsi que par le ministère de l'Éducation nationale, de l'Enseignement supérieur et de la Recherche, s'insère dans le programme de travail du Comité technique international de l'ISO TC46/SC8.

Ses experts, issus d'horizons et d'établissements divers (collectivités territoriales, universités et organismes de recherche, administrations centrales), participent aussi bien à la création qu'à la mise à jour des normes. Afin de valoriser les outils produits par l'ISO en langue anglaise, ils consacrent une part non négligeable de leur énergie à la traduction en français des normes et/ou à l'élaboration de livres blancs destinés à alimenter la réflexion sur des sujets nouveaux.

\section{LA NORMALISATION, UNE DÉMARCHE FACILITATRICE POUR MESURER, ÉVALUER, COMPARER}

L'Afnor et l'ISO produisent des normes volontaires destinées à servir de base dans les relations entre les partenaires économiques, scientifiques, techniques et sociaux et qui constituent des cadres de référence au travers de l'expression de lignes directrices, de prescriptions techniques ou qualitatives pour des produits, des services ou des pratiques orientés vers l'intérêt général. Elles sont le fruit d'un consensus international qui laisse néanmoins de la place aux spécificités nationales ; elles s'appuient sur des retours d'expériences validés.

Les normes des bibliothèques ont vocation à être appliquées aux bibliothèques et centres de documentation de tous types dans le monde ; et à 
être utilisées pour mener des évaluations à différents niveaux (du local à l'international).

Elles portent des corpus de définitions, des méthodologies de comptage voire d'interprétation adaptées. De fait, leur application permet de partager un vocabulaire, des pratiques et, à condition d'expliciter les contextes de leur intervention, de réaliser des comparaisons entre bibliothèques. Elles mettent en relief les données qui font sens. Elles attirent également l'attention sur des secteurs d'activité naissants et difficiles à cerner. Elles sont accompagnées d'une bibliographie souvent abondante.

Les normes font l'objet d'un examen régulier pour évaluer leur pertinence dans le temps.

PANORAMA DES NORMES POUR LES BIBLIOTHĖQUES

TABLEAU 1

INDICATEURS ET STATISTIQUES :

TROIS RAPPORTS TECHNIQUES

\begin{tabular}{|l|l|l|}
\hline Norme & Nom & Date d'édition \\
\hline IS0/TR 11219:2012 & $\begin{array}{l}\text { Conditions qualitatives et statistiques } \\
\text { fondamentales pour bâtiments } \\
\text { de bibliothèques - Espaces, fonctions } \\
\text { et conception }\end{array}$ & mai 2012 \\
\hline IS0/TR 28118:2009 & $\begin{array}{l}\text { Indicateurs de performance } \\
\text { des bibliothèques nationales }\end{array}$ & avril 2012 \\
\hline IS0/TR 14873:2013 & $\begin{array}{l}\text { Statistiques et indicateurs de qualité } \\
\text { pour l'archivage du Web }\end{array}$ & décembre 2013 \\
\hline
\end{tabular}


TABLEAU 2 TROIS NORMES PHARES COMPLÉMENTAIRES

\begin{tabular}{|l|l|l|l|}
\hline Norme & Nom & Dernière édition & Édition française \\
\hline NF/ISO 2789 & $\begin{array}{l}\text { Statistiques internatio- } \\
\text { nales de bibliothèques }\end{array}$ & $5^{\text {e éd. : août 2013 }}$ & mai 2014 \\
\hline NF/IS0 11620 & $\begin{array}{l}\text { Indicateurs de perfor- } \\
\text { mance des bibliothèques }\end{array}$ & $3^{\text {e éd. : mai 2014 }}$ & avril 2015 \\
\hline IS0 16439 & $\begin{array}{l}\text { Methods and procedures } \\
\text { for assessing the impact } \\
\text { of libraries }\end{array}$ & $1^{\text {re éd. : avril 2014 }}$ & $\begin{array}{l}\text { Livre blanc : } \\
\text { Qu'est-ce qui } \\
\text { fait la valeur des } \\
\text { bibliothèques ?a }\end{array}$ \\
\hline $\begin{array}{l}\text { a. Afnor, février 2016. [En ligne] : http://portailgroupe.afnor.fr/public_espacenor- } \\
\text { malisation/AFNORCN46-8/index.html >. }\end{array}$ \\
\hline \multicolumn{3}{|l}{} \\
\hline
\end{tabular}

Par ailleurs, un nouveau groupe de travail ISO est constitué en 2016 afin d'élaborer une nouvelle norme sur la mesure de la qualité appliquée aux bibliothèques nationales.

\section{Les apports des normes $\mathbf{2 7 8 9}$ et $\mathbf{1 1 6 2 0}$}

La norme ISO 2789 Statistiques internationales des bibliothèques est née en 1974 sous l'impulsion de l'Unesco. Elle constitue le socle pour le recueil des données des bibliothèques et la présentation des statistiques ; dès 1976, l'ESGBU s'est appuyé sur cette norme qui sert aussi de référence aux enquêtes nationales des bibliothèques publiques.

La dernière version fait la part belle aux services développés par les bibliothèques et au numérique. Ses nombreuses définitions ont donc été abondamment renouvelées pour tenir compte des nouvelles réalités des pratiques des usagers et des bibliothèques hybrides ; sa structure a été profondément remaniée et simplifiée puisque services et usages sont traités avant les collections.

Ses définitions sont les plus précises possible mais laissent parfois transparaître la difficulté de l'exercice ; ainsi, si la norme définit précisément 
ce qu'est une entrée, ce qu'est un accès en réservant la notion au numérique, s'agissant des usagers, les catégories sont nombreuses et la définition d'un usager actif (article 2.2.3) recommande de compter séparément les usagers actifs non-inscrits identifiés au cours d'enquêtes par exemple. La norme ISO 11620 a pour objectif de promouvoir l'emploi d'indicateurs de performance caractérisant la qualité des services de bibliothèques, et de préciser comment procéder à la mesure et comment l'analyser. Les indicateurs proposés visent à aider les bibliothèques à produire des données pour le pilotage et l'attribution des moyens, mettant en exergue les notions d'efficacité et d'efficience.

La nouvelle version développe un panel d'indicateurs fortement augmenté (une vingtaine d'indicateurs supplémentaires par rapport à la version précédente), toutefois la dimension du numérique reste encore limitée compte tenu des difficultés persistantes de comptage ; les indicateurs de coût sont actualisés.

L'annexe A dresse la liste des indicateurs de performance traités dans la norme en présentant pour chacun l'objectif assigné à sa construction et à son analyse, selon quatre axes :

- ressources, accès et infrastructure pour en mesurer l'adéquation et la disponibilité (personnel, collections, places de consultation, etc.) ;

- utilisation des ressources, des services (prêts, téléchargements, utilisation des équipements, etc.) ;

- efficience des ressources, des services (coût par prêt, délai de traitement des documents, d'acquisition, etc.) ;

- potentialités et développement pour mesurer l'investissement de la bibliothèque dans les services et ressources émergentes (pourcentage de personnel affecté à la fourniture de documents électroniques, etc.).

L'annexe B précise les objectifs des indicateurs, leur domaine d'application, définition, méthode, interprétation et facteurs ayant une incidence sur l'indicateur, sources, indicateurs associés. 


\section{La norme 16439 et le livre blanc}

La norme 16439 Méthodes et procédures pour évaluer l'impact des bibliothèques a l'ambition d'aider les bibliothèques à prouver leur valeur, tant pour les individus que pour la société, à la collectivité et aux institutions qui les financent, dans un contexte marqué par la rétraction des budgets publics où cette valeur n'est plus considérée comme allant de soi.

Elle a l'ambition de doter les professionnels mais aussi les décideurs et financeurs, d'un éventail d'outils et de méthodes adaptés pour mesurer l'impact des bibliothèques sur leur environnement et contribuer ainsi à l'émergence d'éléments de réflexion pour éclairer les choix politiques. Un des atouts de cette norme est de préciser le vocabulaire de façon à faire émerger un langage commun sur le sujet.

Bien-être collectif, qualité de vie, impacts économiques font partie des notions prises en compte dans la norme ISO 16439, ainsi que la dimension " évaluation des politiques publiques » (lecture publique, information scientifique et technique, etc.). Son objectif est de construire des indicateurs qui parlent aux élus et aux autorités qui allouent les ressources nécessaires à la création et au fonctionnement des bibliothèques.

Pour valoriser cette norme et favoriser l'éclosion en France d'expérimentations, la CN46-8 de l'Afnor a fait paraître un livre blanc présenté le 24 mars 2016 dans une journée d'étude intitulée « Qu'est-ce qui fait la valeur des bibliothèques ? Une nouvelle norme pour mesurer leur impact. » ${ }^{9}$ Avant d'aborder les méthodologies pour mesurer la satisfaction des utilisateurs, l'impact sociétal des bibliothèques et enfin leur impact économique, les auteurs du livre blanc proposent une synthèse de la définition de l'impact et présentent les outils de mesure à disposition. Mesurer l'impact conduit à adopter une approche systémique de l'influence de la bibliothèque dans un ensemble de relations ; cela permet d'évaluer les conséquences de l'action de la bibliothèque sur la société, les individus, les groupes, le quartier, les usagers et les non-usagers.

L'impact est apprécié au travers de plusieurs types de données : «les données induites (inferred) des statistiques habituellement collectées par la bibliothèque dans le cadre de ses activités et suivies par la bibliothèque ;

9. < http://www.enssib.fr/JE-valeur-des-bibliotheques > 
les données sollicitées (solicited) : il s'agit d'informations ou de données que l'on va collecter auprès des usagers au moyen de questionnaires ou d'entretiens ; les données observées (observed) qui sont le fruit d'observations dans le cadre de méthodologies inspirées de l'ethnologie. »10 Le livre blanc intègre également trois synthèses d'études étrangères récentes et une bibliographie augmentée.

10. Afnor CN46-8, Qualité-Statistiques et évaluation des résultats, Qu'est-ce qui fait la valeur des bibliothèques ?, février 2016. [En ligne] : < http://portailgroupe.afnor.fr/public_espacenormalisation/AFNORCN46-8/Livre\%20Blanc\%20fev2016.pdf >. 


\section{2}

LIBRES RÉFLEXIONS AU SUJET DES MÉTHODES ET DES ENJEUX DE L'IMPACT ÉCONOMIQUE ET SOCIÉTAL DES BIBLIOTHĖQUES ${ }^{1}$

par

Françoise

Benhamou

Commençons par une analogie. On cite souvent André Malraux, ministre de la Culture, clôturant en ces termes le livre qu'il consacrait à l'esthétique du cinéma : "Par ailleurs, le cinéma est une industrie ». On pourrait écrire de même " par ailleurs, la bibliothèque est un acteur de la vie économique ». Sous bien des aspects, la bibliothèque, acteur de la vie intellectuelle et culturelle, s'avère être aussi un acteur économique. On débouche alors immédiatement sur un paradoxe. Il est demandé à la bibliothèque d'agir en acteur économique, mais l'essentiel des services qu'elle propose relève de la sphère non-monétaire. Il faut ajouter ici un élément de contexte. Celui de la multiplication des études d'impact de la culture, et du regard qu'il convient de porter sur leurs méthodologies et leurs résultats. Celles qui portent sur les bibliothèques ne sauraient être analysées sans qu'il soit porté une attention particulière à l'appétit soudain pour l'économie qui traverse les mondes de la culture.

Après une revue des données et des concepts qui sous-tendent les études d'impact des bibliothèques ( $1^{\text {re }}$ partie), on montrera que deux approches complémentaires coexistent ( $2^{\mathrm{e}}$ partie), qui aboutissent à des résultats ( $3^{\mathrm{e}}$ partie) que l'on discutera à la lumière de la question plus générale de l'appréciation des retombées de la culture sur l'économie (4e partie).

\section{DES ACTIVITÉS ÉCONOMIQUES DIVERSIFIÉES}

Comment repérer simplement les activités économiques de la bibliothèque? En premier lieu, la bibliothèque emploie, achète et propose des services.

1. Cet article reprend en partie une présentation faire au colloque de l'Association des directeurs de la documentation et des bibliothèques universitaires (ADBU), septembre 2014. 
On comptait 6157 emplois dans la fonction publique d'État, filière bibliothèques, toutes catégories confondues, en février $2012^{2}$. Concernant les effectifs en bibliothèque territoriale, on dispose de deux estimations : 33000 à 34000 agents territoriaux affectés en bibliothèque en 2010 selon le Centre national de la fonction publique territoriale (CNFPT) ${ }^{3}$, et 36467 agents du cadre d'emploi bibliothèques et patrimoine d'après les statistiques du ministère de la Culture.

\section{LES BIBLIOTHÉCAIRES PRODUCTEURS POUR LES USAGERS...}

Ces agents produisent des services. Quels sont les outputs, essentiellement publics ? Le bibliothécaire participe à la formation : il prête et conseille. Il contribue à l'amélioration de la recherche en accroissant l'efficacité de l'activité scientifique des chercheurs. S'il sert ainsi à la recherche, il mène une activité scientifique et produit des métadonnées.

Il agit sur le marché éditorial : il achète des livres en format papier et en format électronique ; on note que les abonnements à des revues académiques brident la possibilité d'acquérir des livres et fragilisent notamment les marchés des livres scientifiques et de sciences humaines.

Le bibliothécaire est un acteur des territoires dans lesquels son activité s'inscrit ; mais l'impact de la bibliothèque s'étend au-delà de ces territoires : le service éducatif et le service de loisirs constituent une offre qui produit une amélioration du bien-être social non seulement des usagers réguliers ou occasionnels, mais aussi des non-usagers qui profitent indirectement des effets bénéfiques de la bibliothèque pour l'amélioration du « vivre ensemble ».

2. Pierre Carbone, Dominique Arot, Joëlle Claud, Benoît Lecoq, Charles Micol, « Quels emplois dans les bibliothèques ? État des lieux et perspectives ", Rapport n²012-020, Paris, Inspection générale des bibliothèques, 2013. [En ligne] : < http://cache.media.enseignementsup-recherche.gouv. fr/file/2013/66/7/Rapport_IGB_2013-005_Des_indicateurs_documentaires_pour_les_sites_universitaires_283667.pdf $>$. Le rapport précise le pyramidage observé sur l'ensemble de la filière : $34 \%$ du personnel en catégorie $\mathrm{A}$, près de $27,5 \%$ en catégorie $\mathrm{B}$, et plus de $38,5 \%$ en catégorie $\mathrm{C}$.

3. L'Observatoire de l'emploi, des métiers et des compétences de la fonction publique territoriale publie annuellement une note de conjoncture Tendances de l'emploi territorial (données nationales à partir des déclarations d'un échantillon représentatif de collectivités). 


\section{ET MÊME POUR LES NON-USAGERS...}

Il faut distinguer en effet quatre sources de valeur : d'un côté, la valeur d'usage*, et, d'un autre côté, les valeurs de "non-usage »*, qui comprennent la valeur d'option, la valeur d'existence, et la valeur de transmission. Dans les trois derniers cas, il n'y a pas d'usage du service, du moins dans l'immédiat. La valeur d'option consiste en la possibilité de se rendre dans une bibliothèque à un horizon non défini. La valeur d'existence est la satisfaction de savoir qu'existe, dans son pays ou ailleurs, un établissement où, paradoxalement, on n'a aucune intention de se rendre. La valeur d'héritage ou de transmission désigne la jouissance que tirent les citoyens à transférer un patrimoine, un lieu culturel, aux générations futures. Ces trois valeurs, présentes en matière de bibliothèques, ne se traduisent pas par un prix et ne donnent pas lieu à consommation immédiate. Elles peuvent justifier, de ce fait, le recours à des financements publics.

On rejoint ici la problématique des externalités, c'est-à-dire des effets d'une action dont le coût n'a pas été supporté par ceux qui en profitent. Celles-ci sont mal prises en compte : si les chercheurs sont des utilisateurs des services offerts, dans une BU par exemple, leurs recherches contribuent à l'innovation au sein de l'économie, à court et surtout à long terme. L'externalité correspond à l'avantage dont profitent des entreprises de divers secteurs, qui bénéficient des avancées de la recherche menée avec l'appui des services de la bibliothèque, sans qu'elles aient eu à financer le fonctionnement de celle-ci.

\section{LES DEUX APPROCHES COMPLÉMENTAIRES}

Il faut distinguer deux approches complémentaires en matière d'évaluation de l'empreinte économique de la bibliothèque, l'étude d'impact et l'analyse coût-bénéfices. 


\section{LES ÉTUDES D'IMPACT}

La méthodologie semble a priori simple : évaluer les retombées directes et indirectes de la bibliothèque. Les premières sont associées à la visite et au temps passé dans les murs, et incluent les dépenses de transport ou de restauration des usagers. Les salariés de la bibliothèque dépensent les revenus qu'ils reçoivent. Afin d'évaluer cet « impact », on a coutume d'appliquer un coefficient multiplicateur à la dépense initiale des usagers. Le multiplicateur est généralement issu d'une évaluation de l'impact économique de situations supposées analogues. Il faut aussi prendre en compte les coûts (fonctionnement, entretien, etc.) afin d'aboutir à des retombées nettes. La bibliothèque dépense en effet son budget en abonnements, commandes de livres, achats de biens de consommation ou de biens intermédiaires : papier, imprimantes, etc. Ces dépenses elles-mêmes ont un impact économique, mais certaines peuvent s'adresser à des entreprises non françaises ; il faut alors les défalquer du total, car elles constituent des fuites du circuit économique, en quelque sorte. Dans le cas contraire, elles se diffusent par tours successifs dans l'économie locale.

On aperçoit vite les limites de ces études : difficulté d'estimation des dépenses induites, caractère peu fondé du choix d'un coefficient multiplicateur, risque de surestimation de l'impact qui suscite plus d'effets d'éviction que de création nette de richesse : ce que le visiteur de la bibliothèque dépense à l'occasion de sa visite n'est que le simple déplacement d'une dépense qu'il aurait effectuée pour un autre service ou pour l'achat d'un autre bien. Mais on peut aussi faire l'hypothèse d'une sous-estimation sur un autre terrain, car ces études font peu de cas des valeurs de non-usage, fondamentales en économie de la culture ${ }^{4}$.

\section{LES ANALYSES COÛT-BÉNÉFICES}

Le principe en œuvre, dans les analyses coût-bénéfices, consiste en le repérage des coûts proprement dits ainsi que des " coûts d'opportunité » d'un côté, et des bénéfices de l'autre côté. Aux coûts proprement dits,

4. Pour plus de détails, voir Françoise Benhamou, L'économie de la culture, $7^{\mathrm{e}}$ éd., Paris, La Découverte, 2011 (coll. Repères ; 192). 
il convient en effet d'ajouter ce qu'un autre usage des fonds publics aurait éventuellement permis de gagner : le coût d'opportunité est en quelque sorte le coût du renoncement à un investissement ou à une dépense alternatifs, ou à une affectation des ressources à un usage plus porteur (le critère de la comparaison pouvant être, par exemple, le nombre d'emplois créés, ou encore les externalités positives générées). Du côté des bénéfices, il faut distinguer les éventuels bénéfices commerciaux de ce que l'on pourrait qualifier de bénéfices sociaux. Les premiers résultent de ce que la bibliothèque peut monétiser certains services ou vendre des produits dérivés. Les seconds renvoient au fait que la bibliothèque est un lieu de recherche et de travail, qu'elle apporte ses services à la communauté : salle de lecture, documentation, site web, événements et expositions, services éducatifs. La bibliothèque a une fonction citoyenne d'inclusion sociale, de lieu de rendezvous, d'espace de convivialité. Convoquant un concept emprunté au sociologue Ray Oldenburg ${ }^{5}$, Robert Putnam voit la bibliothèque comme " un troisième lieu » (Third Place Library) à côté du foyer et du bureau. Le troisième lieu est neutre, ouvert à tous, indifférent aux inégalités sociales, adapté à la conversation, à l'échange informel entre tous les membres de la communauté, convivial et fréquenté par des habitués, et il procure des opportunités de rencontres. On s'éloigne ainsi de la bibliothèque centrée sur le livre et le document pour glisser vers une bibliothèque pourvoyeuse d'autres services (aide à la recherche d'emploi, salons de lecture numérique) ${ }^{6}$.

\section{L'évaluation contingente}

Les méthodes dites d'évaluation contingente* consistent en l'identification des services offerts, puis en l'évaluation pour chaque service, grâce à des enquêtes et des questionnaires, du consentement à payer

5. Ray Oldenburg, The Great Good Place: Cafes, Coffee Shops, Bookstores, Bars, Hair Salons, and Other Hangouts at the Heart of a Community, 4éd., Boston, Marlowe \& Co, 1999.

6. Dans les écrits de Ray Oldenburg, le troisième lieu est le café. Voir notamment : Mathilde Servet, " Les bibliothèques troisième lieu : une nouvelle génération d'établissements culturels ", Bulletin des bibliothèques de France, 2010, $\mathrm{n}^{\circ} 4$, pp. 57-63. [En ligne] : < http://bbf.enssib.fr/ consulter/bbf-2010-04-0057-001 > et Françoise Benhamou, Le livre à l'heure numérique. Papier, écrans. Vers de nouveaux vagabondages, Paris, Le Seuil, 2014. 
de l'usager. On désigne ainsi la somme maximale que l'usager est prêt à payer pour un service. Cette méthode, communément utilisée en économie, revêt nombre d'inconvénients. Elle est tributaire des comportements stratégiques de la part des répondants, peu enclins à révéler un consentement à payer plus élevé que le prix (éventuellement nul) du service offert. C'est pourquoi il est fréquent de le remplacer par le consentement à accepter, soit la somme minimale que l'usager accepte de recevoir en échange du renoncement à un service. Concrètement, il peut s'agir de l'empêchement d'accéder à la salle de lecture, évalué par une proxy : le prix auquel on est prêt à vendre sa carte d'accès. On observe en général que le consentement à renoncer est supérieur au consentement à payer : jouent à la fois une forme d'aversion au risque (en l'occurrence le risque de perdre la possibilité de l'accès) et la sensibilité à la contrainte de budget.

On évalue enfin le coût marginal de remplacement, c'est-à-dire l'ensemble des coûts additionnels que l'on est prêt à assumer afin de remplacer un service par un service alternatif. Si l'on multiplie ces valeurs par le nombre des usagers, on obtient une évaluation de la valeur conférée à la bibliothèque. Prenons l'exemple des services web, que l'on peut utiliser aussi bien sur place qu'à distance. Ils sont générateurs d'économies de temps et de déplacement, que l'on peut évaluer par le nombre de visites du site, le pourcentage de visites physiques évitées grâce à des visites virtuelles et le coût moyen de déplacement, sachant que le Web permet de surcroît d'élargir le nombre des bénéficiaires des services.

\section{ÉTABLIR LE RETOUR SUR INVESTISSEMENT : DEUX CAS CONCRETS}

Bruce Kingma et Kathleen McClure ${ }^{7}$ appliquent ces méthodes au cas de la Syracuse University Library, afin d'évaluer le retour sur investissement

7. Bruce Kingma et Kathleen McClure, "Lib-Value: Values, Outcomes, and Return on Investment of Academic Libraries, Phase III: ROI of the Syracuse University Library", College \& Research Libraries, January 2015, 76 (1). [En ligne] : < http://crl.acrl.org/content/early/2014/01/17/crl13485.full.pdf + html >. 
(Return on Investment ou ROI en anglais) de la bibliothèque ${ }^{8}$. Cette dernière compte à la date de l'étude 3,8 millions de livres, 74500 revues et $900000 €$-books, pour un budget annuel de fonctionnement de 17 millions de dollars. La valeur économique de l'établissement est évaluée à 70,2 millions de dollars. La prise en compte tout à la fois des visites sur place et des consultations à distance permet d'établir le ROI à 4,13 (obtenu en divisant la valeur économique par le budget) ${ }^{9}$.

Andrew Tessler ${ }^{10}$ s'attelle à l'évaluation de la British Library. Le tableau 1 fait état des résultats pour le site web, et le tableau 2 indique la valeur de l'établissement dans son ensemble. Le site web est évalué sur la base du temps économisé et de la valeur de ce temps (le salaire horaire moyen) ; le résultat global prend en compte l'ensemble des coûts (subventions, donations, externalités sur l'environnement), et des bénéfices (revenus, salles de lecture, offre documentaire, services web, services liés à la réserve, expositions, événements, services éducatifs et valeurs de non-usage), et aboutit à un ratio bénéfices/coûts de 4,9. Dans cette étude, l'usage des salles de lecture est évalué à 70 millions de $£$ par an, dont plus de 20 millions pour le Business \& IP Centre, les services web à 19,5 millions par an ; la contribution au secteur de l'éducation à travers la UK Research Reserve à 5,4 millions par an, et la valeur (d'existence) de la British Library pour la société (pour les non-usagers) à 413 millions par an.

8. Le site < http://libvalue.cci.utk.edu/ > recense les principales études basées sur cette méthodologie.

9. Bruce Kingma et Kathleen McClure, "Lib-Value: Values, Outcomes, and Return on Investment of Academic Libraries, Phase III: ROI of the Syracuse University Library”, op. cit.

10. Andrew Tessler, Economic Valuation of the British Library: A report for the British Library, Oxford, Oxford Economics, janvier 2013. 
TABLEAU 1 VALEUR DU SITE WEB DE LA BRITISH LIBRARY

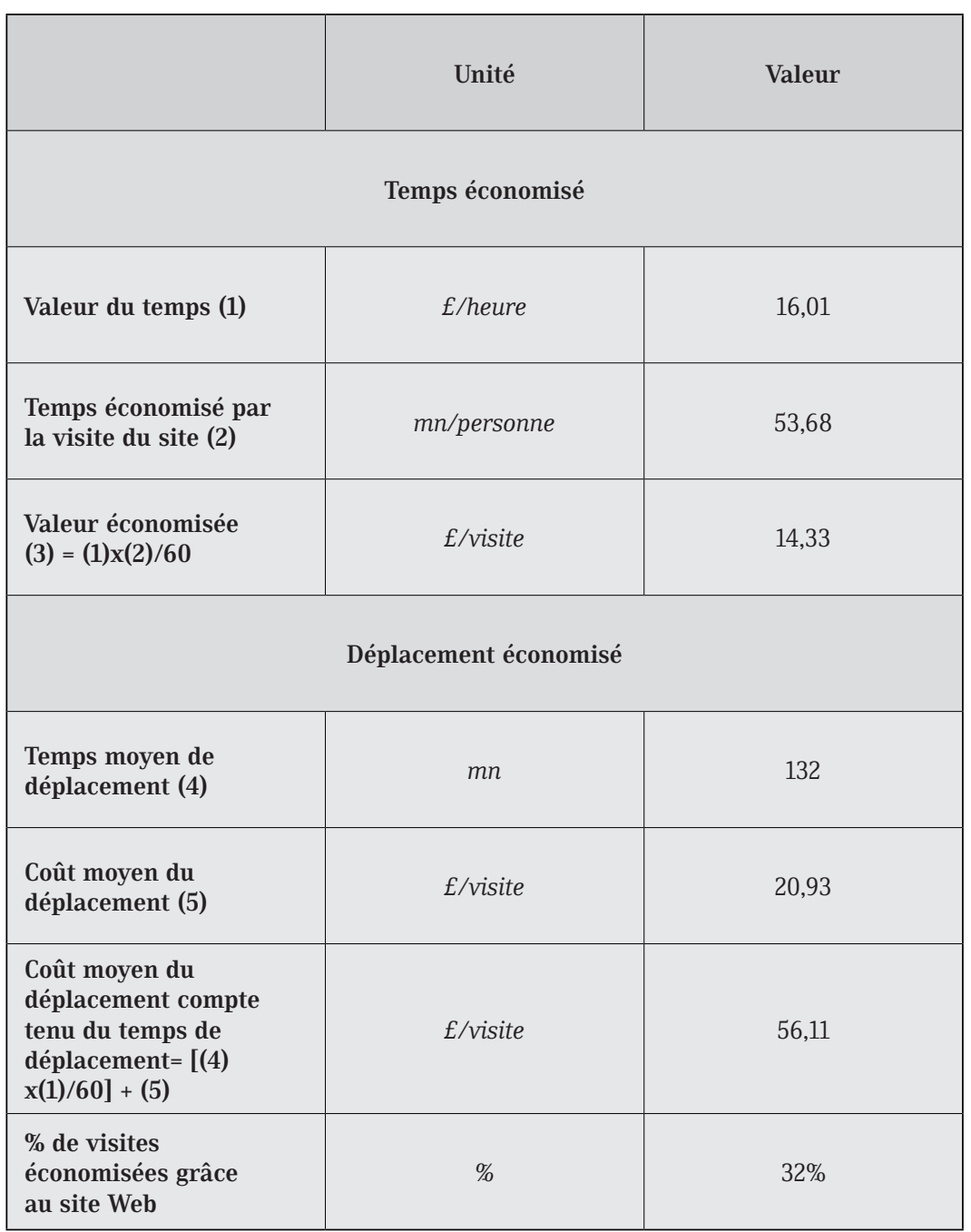

Source: Andrew Tessler, Economic Valuation of the British Library: A report for the British Library, Oxford, Oxford Economics, janvier 2013. 
CALCUL DU RATIO COÛTS-AVANTAGES

POUR LA BRITISH LIBRARY, 2011-2012, Mf

\begin{tabular}{|c|c|c|c|}
\hline \multicolumn{2}{|c|}{ Coûts } & \multirow{2}{*}{$\begin{array}{l}\text { Bénéfices } \\
\text { Revenus }\end{array}$} & \multirow{2}{*}{$\begin{array}{l}\mathrm{M} £ \\
11,5\end{array}$} \\
\hline Subventions & 101,9 & & \\
\hline Donations & 5,9 & Salles de lecture & 70,1 \\
\hline \multirow[t]{6}{*}{$\begin{array}{l}\text { Externalités sur } \\
\text { l'environnement }\end{array}$} & 0,5 & $\begin{array}{c}\text { Offre } \\
\text { documentaire }\end{array}$ & 5,0 \\
\hline & & Services web & 19,5 \\
\hline & & $\begin{array}{l}\text { Services liés } \\
\text { à la réserve }\end{array}$ & 5,4 \\
\hline & & $\begin{array}{l}\text { Expositions, } \\
\text { événements }\end{array}$ & 2,5 \\
\hline & & Services éducatifs & 0,7 \\
\hline & & Non-usage & 412,8 \\
\hline Total (1) & 108,3 & Total (2) & 527,3 \\
\hline \multicolumn{2}{|c|}{ Bénéfices nets des coûts (2)-(1) } & 419,0 & \\
\hline \multicolumn{2}{|c|}{ Ratio coûts-avantages (2)/(1) } & 4,9 & \\
\hline
\end{tabular}

Source : Ibid. 


\section{UNE ANALYSE CRITIQUE ET QUELQUES PERSPECTIVES : L'EXEMPLE DES BU DU ROYAUME-UNI}

Nombreuses sont les études qui tentent ainsi l'exercice de l'évaluation des bénéfices induits de l'existence de la bibliothèque. L'une d'entre elles porte sur la valeur des services des bibliothèques aux chercheurs et à la recherche au Royaume-Uni en 2011. D'emblée, l'analyse quantitative se heurte à la faible disponibilité des données statistiques sur l'apport et les caractéristiques des bibliothèques. Dans le cas des bibliothèques universitaires, la collecte de données qualitatives à partir d'entretiens auprès de chercheurs, des vice-présidents des universités en charge de la recherche et de l'innovation, du bureau d'aide à la recherche, etc., dans neuf institutions, a permis de démontrer la forte contribution des BU aux performances individuelles et institutionnelles en matière de recherche ${ }^{11}$. Le tableau 3 fait état des principaux aspects qu'il conviendrait de développer dans ce type d'évaluation, l'apport de la bibliothèque étant abordé sous trois de ses aspects : qualité de la recherche, productivité des chercheurs, et rayonnement de l'institution.

Au-delà d'une évaluation quantitative, toujours contestable, plusieurs éléments peuvent être mis en avant : gain en temps, relation entre publications et moyens affectés à la BU, toutes choses égales par ailleurs, relation entre fréquentation (physique ou à distance) et productivité de la recherche. Sans doute faudrait-il introduire des paramètres concernant les disciplines, l'usage des ressources documentaires et le type de ressources utilisées variant drastiquement entre sciences dures et sciences humaines.

11. Research Information Network (RIN) et Research Libraries United Kingdom (RLUK), The value of libraries for research and researchers, mars 2011. [En ligne]: < http://www.rluk.ac.uk/wpcontent/uploads/2014/02/Value-of-Libraries-full-report-.pdf >. 


\section{TABLEAU 3}

APPORT DES BU AUX PERFORMANCES INDIVIDUELLES ET INSTITUTIONNELLES EN MATIÈRE DE RECHERCHE

\begin{tabular}{|c|c|c|}
\hline & $\begin{array}{l}\text { Apport de la bibliothèque } \\
\text { non monétisable }\end{array}$ & Aspect monétisable \\
\hline $\begin{array}{c}\text { Qualité } \\
\text { de la recherche }\end{array}$ & $\begin{array}{l}\text { Aide aux institutions } \\
\text { pour le recrutement des } \\
\text { meilleurs chercheurs }\end{array}$ & \\
\hline $\begin{array}{l}\text { Productivité } \\
\text { des chercheurs }\end{array}$ & $\begin{array}{l}\text { Espaces réservés aux } \\
\text { chercheurs, meilleur } \\
\text { environnement de travail } \\
\text { Facilité d'accès à des } \\
\text { contenus d'excellente } \\
\text { qualité } \\
\text { Promotion de nouveaux } \\
\text { modèles de communica- } \\
\text { tions entre chercheurs }\end{array}$ & $\begin{array}{l}\text { Aide aux chercheurs } \\
\text { pour l'obtention de sub- } \\
\text { ventions et de contrats } \\
\text { de recherche }\end{array}$ \\
\hline $\begin{array}{l}\text { Rayonnement } \\
\text { de l'institution }\end{array}$ & $\begin{array}{l}\text { Promotion et exploitation } \\
\text { des nouvelles technologies } \\
\text { Archives institutionnelles } \\
\text { permettant l'accroisse- } \\
\text { ment de la visibilité } \\
\text { de l'institution } \\
\text { et la valorisation de son } \\
\text { profil de recherche } \\
\text { Ouverture des biblio- } \\
\text { thèques vers l'extérieur } \\
\text { contribuant au lancement } \\
\text { d'initiatives dans toute } \\
\text { l'institution } \\
\text { Bibliothèques comme } \\
\text { manifestation physique de } \\
\text { la valeur des études et du } \\
\text { savoir }\end{array}$ & \\
\hline
\end{tabular}


La vogue des évaluations laisse perplexe ${ }^{12}$. D’un côté, il est légitime et souhaitable de procéder à des évaluations des bénéfices engendrés par une dépense effectuée sur fonds publics en quasi-totalité. Mais l'évaluation conduit à des approximations et à des biais et porte en elle la tentation de la comparaison de la dépense avec d'autres usages des fonds publics plus créateurs d'emplois et de richesses dans le court terme. Certes, la mesure permet de prendre distance avec l'idée de démesure associée par certains à la politique culturelle. Mais la propension à l'économisme risque de masquer l'apport véritable de la bibliothèque, apport de long terme qui inclut surtout des éléments qualitatifs, qui vont de la qualité de la citoyenneté à la large palette des services offerts aussi bien aux plus humbles qu'aux chercheurs de haut niveau.

12. Françoise Benhamou et Philippe Chantepie, «Culture \& économie : chiffres et cryptes », Bulletin des bibliothèques de France, 2016, $\mathrm{n}^{\circ}$ 8, pp. 9-18. [En ligne] : < http://bbf.enssib.fr/consulter/ bbf-2016-08-0008-001 >. 


\section{PARTIE II}

\section{MÉTHODES ET OUTILS}

1. CONSTRUIRE UN TABLEAU DE BORD POUR LA BIBLIOTHĖQUE ? par Marc Maisonneuve

++++++++++++++++++++++++++++++++++++++++++++++++++++++++++++++

2. ÉVALUER L'IMPACT DE SA BIBLIOTHĖQUE SUR LE TERRITOIRE : MESURER LA ZONE DE CHALANDISE OU DE RAYONNEMENT DE SA BIBLIOTHÈQUE par Emmanuelle Asselin

3. MESURER LA VALEUR SOCIÉTALE DE LA BIBLIOTHĖQUE PUBLIQUE : DÉFINITIONS, CRITĖRES ET MÉTHODES par Frank Huysmans et Marjolein Oomes $+++++++++++++++++++++++++++++++++++++++++++++++++++++++++++++++$

4. ARRÊTER, COMMENCER, CONTINUER : ÉVALUER POUR DÉCIDER par Nathalie Clot

$++++++++++++++++++++++++++++++++++++++++++++++++++++++++++++++++$

5. ÉVALUER ET INSPIRER LES TERRITOIRES DE DEMAIN par Marie-Christine Jacquinet

$++++++++++++++++++++++++++++++++++++++++++++++++++++++++++++++++$

6. MESURER LA FRÉQUENTATION DE 121 ÉTABLISSEMENTS : L'EXEMPLE DE LA BIBLIOTHĖQUE DÉPARTEMENTALE DU VAL D'OISE par Cécile Avallone 


\section{1}

\section{CONSTRUIRE UN TABLEAU DE BORD POUR LA BIBLIOTHĖQUE ?}

par

Marc

Maisonneuve

Périodiquement absorbées par la production de rapports d'activité, les bibliothèques disposent d'une véritable culture statistique. La charge de préparation de ces rapports à finalité administrative est toutefois telle qu'elle semble souvent décourager l'élaboration de tableau de bord à visée plus opérationnelle. Plusieurs mouvements sociétaux accroissent aujourd'hui la pression sur les chefs de service sommés de rendre compte du bon emploi des budgets publics et de la bonne gestion de leur établissement. De plus en plus, la production de statistiques semble associée à un fort enjeu de communication visant à sécuriser l'affectation des moyens de fonctionnement. Par choix ou par contrainte, la construction du tableau de bord de la bibliothèque s'inscrit ainsi dans un cadre plus dynamique avec des enjeux de relativement court terme. En pratique, la ou le responsable de la bibliothèque se trouve confronté.e à de multiples questions : comment positionner ce tableau de bord ? À quelle fréquence le publier ? Quels indicateurs retenir pour répondre aux attentes de ses destinataires ?

\section{DE L'ESGBU ET DU RAPPORT SLL AU TABLEAU DE BORD URBAIN}

Créé en 1976, l'ESGBU ${ }^{1}$ visait la fourniture de données statistiques à l'administration centrale afin de lui permettre de répartir au mieux les moyens de fonctionnement. Depuis 1969, le ministère de la Culture collecte des données chiffrées lui permettant de suivre l'activité des bibliothèques territoriales, en exécution du contrôle technique qu'exerce l'État. Dans un cas comme dans l'autre, la production du rapport statistique s'inscrit dans le cadre d'une remontée d'information d'une bibliothèque disposant d'une mission locale à destination d'un service ministériel. Il n'y a pas dans ce processus à proprement parler d'échanges. La finalité de ces rapports

1. Voir la définition dans le dictionnaire de l'Enssib : < http://www.enssib.fr/le-dictionnaire/esgbu >. 
statistiques s'inscrit dans une logique de pilotage ; ils n'impactent que fort peu l'allocation des moyens, décidée localement. Même s'ils demeurent une source précieuse d'évaluation de sa propre bibliothèque, ces états statistiques ne sont guère adaptés à l'enjeu du moment : sécuriser l'obtention des budgets qui permettront à l'établissement de remplir ses missions dans de bonnes conditions et avec de bons résultats. Autre travers de ces rapports : volumineux, structurés mais arides, ils ne se prêtent guère à des actions de communication.

Au-delà de l'inscription du tableau de bord dans une démarche offensive de sécurisation des budgets, plusieurs autres mouvements sont porteurs d'attentes nouvelles concernant le suivi de l'activité de la bibliothèque : " ville intelligente »* ou Smart City en anglais, ouverture des données publiques, amélioration de la gouvernance.

\section{DES BIBLIOTHĖQUES « INTELLIGENTES ॥ OFFRANT DES SERVICES INNOVANTS}

Selon Wikipedia, une « ville intelligente » désigne « une ville utilisant les technologies de l'information et de la communication pour améliorer la qualité des services urbains ou encore réduire ses coûts ». Elle intègre une gouvernance participative, le citoyen étant appelé à être acteur de la vie démocratique.

\section{Connaître la disponibilité des places en temps réel}

Développée initialement pour la Bpi, une application mobile a ensuite été adaptée pour signaler aux étudiants les places disponibles dans les BU parisiennes. Cet outil mobile illustre le concept de ville intelligente et même de bibliothèques intelligentes. Le nombre de places assises disponibles dans sa bibliothèque constitue ainsi un indicateur fort apprécié des usagers lorsqu'il est produit en temps réel et immédiatement disponible sur un smartphone. Calculé à la semaine, au mois ou à l'année, cet indicateur peut également servir d'appui à un discours structuré quant à la saturation des locaux ou à l'insuffisance du nombre de places assises. Il viendra utilement alimenter les échanges avec les élus lors de la préparation budgétaire. 


\section{L'OUVERTURE DES DONNÉES PUBLIQUES POUR FAVORISER L'APPROPRIATION CITOYENNE DES BIBLIOTHĖQUES}

La Déclaration des droits de l'homme et du citoyen du 26 août 1789 indique que "la société a le droit de demander compte à tout agent public de son administration ». Mises à la disposition du citoyen, les données produites par les services publics contribuent à ce devoir de transparence. Elles sont également précieuses pour engager une concertation éclairée avec les citoyens, pour dynamiser la vie démocratique locale ou pour stimuler l'innovation avec notamment le développement de nouveaux services numériques utiles au public. L'Unesco et l'IFLA indiquent ${ }^{2}$ que les bibliothèques se doivent " d'assurer l'accès des citoyens aux informations de toutes catégories issues des collectivités locales » et de contribuer au " progrès d'une société démocratique en donnant aux individus accès à une large gamme de savoirs, d'idées et d'opinions ». La ville de Paris s'est engagée dans ce mouvement de libération des données ; concernant la lecture publique, elle propose ainsi le téléchargement des titres les plus prêtés dans les bibliothèques de prêt, des titres les plus réservés... et le catalogue de tous les documents ${ }^{3}$.

Le mouvement de l'open data introduit ainsi de nouvelles dimensions dans la production de statistiques. Il dépasse la simple communication pour approcher un enjeu d'appropriation de la bibliothèque par les citoyens.

\section{LA CONTRIBUTION DES BIBLIOTHĖQUES À UNE MEILLEURE GOUVERNANCE}

Une bonne gouvernance suppose l'organisation d'un dialogue efficace et constructif entre les différents acteurs impliqués dans l'action publique. Elle impose de donner aux citoyens un moyen de contrôle de l'action publique. Elle doit permettre de raffermir le lien entre élus et citoyens. Les taux records d'abstention aux élections et la poussée des extrêmes soulignent les enjeux et l'urgence d'une meilleure gouvernance.

2. Voir le Manifeste de l'Unesco sur la bibliothèque publique. [En ligne] : < http://www.unesco.org/ webworld/libraries/manifestos/libraman_fr.html >.

3. On trouve ainsi 18 jeux de données concernant les bibliothèques sur le site $<$ http://opendata. paris.fr/ >. 
Que peut faire la bibliothèque pour apporter sa contribution à cet ambitieux projet?

Selon 3 axes d'actions, elle peut :

- donner à voir aux citoyens, aux élus et aux agents de la collectivité son bon fonctionnement, en quelques chiffres qui parleront à chacun et qui confirmeront le bon emploi de l'argent public ;

- alimenter tout débat utile sur son positionnement (droit à la culture, droit à l'information, droit à la formation, lutte contre la fracture numérique) ou sur ses choix stratégiques (politiques d'acquisition, offre de services...). Pour créer les conditions d'un débat éclairé sur ces questions, elle devra fournir les informations illustrant ses positionnements actuels et permettant de dimensionner les enjeux associés aux projets qu'elle revendique ;

- être le lieu qui héberge des débats citoyens de qualité, en se positionnant dans un rôle de simple animateur. À ce titre, elle peut réunir et mettre à la disposition de chacun les informations permettant d'éclairer les enjeux.

Dans ces différentes actions en rapport avec l'amélioration de la gouvernance, la bibliothèque verra coexister des opérations récurrentes (produire, publier et rendre accessibles des indicateurs illustrant le bon emploi de l'argent public) et des opérations plus ponctuelles (produire, publier et rendre accessibles des indicateurs démontrant son intervention dans un domaine particulier, plus ou moins investi par elle, afin d'engager un débat sur l'évolution de son positionnement). Nul besoin de souligner que l'articulation avec l'élu référent est une condition essentielle à la réussite de telles opérations! Smart city, open data et amélioration de la gouvernance ont ceci en commun qu'elles aboutissent à concevoir la production d'indicateurs statistiques dans un cadre rénové, plus dynamique et mis au service de la collectivité. Elles introduisent ce que certains qualifient de tableau de bord urbain. Sans prétendre définir ce concept, le tableau de bord urbain pourrait désigner l'ensemble des indicateurs et des données brutes produits par les services publics, destinés tant aux élus qu'aux citoyens, dans un objectif multiforme d'amélioration de la vie quotidienne et d'appropriation collective des services publics. 


\section{LE PÉRIMÈTRE DU TABLEAU DE BORD URBAIN}

Avant de délimiter plus précisément le périmètre du tableau de bord urbain, éliminons la question du positionnement de la bibliothèque dans la préparation de ce tableau de bord. L'Unesco et l'IFLA ${ }^{4}$ incitent à confier à la bibliothèque la mission "d'assurer l'accès des citoyens aux informations de toutes catégories issues des collectivités locales ». Pris au pied de la lettre, cela pourrait aboutir à confier à la bibliothèque le support des outils et des démarches de libération de toutes les données publiques produites par sa collectivité. Ainsi, la bibliothèque pourrait également être le support des indicateurs constituant le tableau de bord urbain provenant des autres services municipaux. Sur le fond rien ne s'oppose à envisager une telle mission pour la bibliothèque mais il faut bien constater que cela n'est pas la droite ligne du positionnement des bibliothèques françaises au sein de leur collectivité. Cela n'est pas non plus un rôle actuellement reconnu aux BU. Nous n'évoquerons donc que la question de la contribution spécifique de la bibliothèque à la constitution de ce tableau de bord.

\section{QUELLES DONNÉES DES BIBLIOTHĖQUES FAUT-IL LIBÉRER ?}

L'expérience des villes et établissements publics de coopération intercommunale (EPCI) ${ }^{5}$ engagés dans l'open data fournit de premières pistes tout à fait intéressantes : libérer le catalogue des collections, libérer la liste des documents les plus empruntés, réservés ou recherchés dans le catalogue, libérer la liste des documents numériques les plus consultés... D’autres pistes méritent probablement d'être explorées : libérer les transactions de prêt de documents après les avoir anonymisées, libérer le fichier anonymisé des usagers avec par exemple le nombre d'usagers domiciliés dans chaque commune et dans chaque quartier... La mise à disposition de ces données aboutira peut-être à de nouvelles analyses des pratiques d'emprunt, à des représentations de la population des usagers mettant à profit la géolocalisation... et à bien d'autres réalisations que la bibliothèque souhaiterait prendre en charge elle-même mais sans avoir les moyens de le faire. Enfin,

4. Voir le Manifeste de l'Unesco sur la bibliothèque publique, op. cit.

5. Autrement dit, communauté de communes et communautés d'agglomération. 
il faut peut-être également envisager de libérer toutes les données élémentaires utilisées pour le calcul des indicateurs composant le tableau de bord urbain. Cela contribuera à sa transparence.

\section{QUELS INDICATEURS LA BIBLIOTHÈQUE DOIT-ELLE PRODUIRE ?}

La sécurisation des moyens de fonctionnement d'une part et les mouvements sociétaux évoqués ci-dessus d'autre part ont permis d'identifier quatre thèmes principaux permettant de structurer la réflexion sur le choix des indicateurs.

\section{Thème 1 : dégager l'impact global de la bibliothèque sur la population}

Indépendamment d'une évaluation de la bonne exécution des missions de la bibliothèque, il s'agit de donner à voir l'impact de la bibliothèque sur la population à desservir. En pratique, il s'agit d'indiquer qui la bibliothèque touche effectivement, avec une approche quantitative (le nombre de personnes ayant utilisé les services de la bibliothèque) et une approche qualitative (le profil des usagers utilisant ces services).

Pour l'approche quantitative, plusieurs indicateurs peuvent rendre compte des principaux usages de la bibliothèque :

- indicateur général : le nombre de personnes inscrites à la bibliothèque au cours de la période ;

- indicateur de la fonction de prêt : le nombre de personnes ayant emprunté au moins un document au cours de la période ;

- indicateur de la fonction « lecture, étude sur place » : une fourchette composée du nombre de personnes venues à la bibliothèque au cours de la période et de ce même nombre diminué du nombre de ceux qui y ont emprunté des documents ;

- indicateur de la fonction de consultation à domicile des ressources numériques : le nombre de personnes ayant consulté à domicile un document numérique (mode connecté) ou ayant emprunté un document numérique (téléchargement). 


\section{FIGURE 1 \\ L'IMPACT DE LA BIBLIOTHĖQUE SUR LA POPULATION EN MARS}

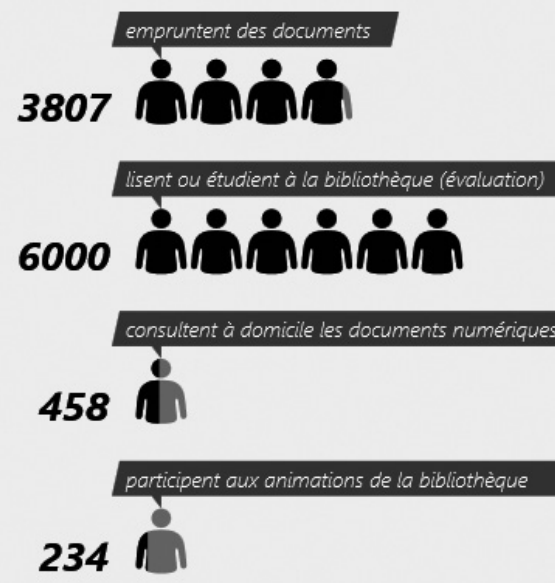

Installée en banlieue, cette bibliothèque semble faire coexister efficacement un modèle traditionnel de bibliothèque (fonction de prêt) et le modèle de bibliothèque $3^{\mathrm{e}}$ lieu. Par contre, son offre numérique peine à conquérir un public significatif. Ses investissements numériques et son offre de ressources numériques sont-ils adaptés aux attentes des jeunes générations et des actifs ? Doit-elle revoir sa politique numérique ou plutôt envisager une ouverture le dimanche?

Arrêtons nous un instant sur la forme retenu pour les indicateurs de la figure 1.

Généralement les indicateurs se présentent sous forme de pourcentages et non sous forme de chiffres bruts, notamment afin de faciliter les comparaisons. Ici, cela aurait consisté à rapporter chacun de ces nombres d'usagers à la population communale. Trois arguments peuvent faire préférer ici les chiffres bruts.

1. Il est plus facile de repérer une évolution mineure au travers de chiffres bruts représentant le nombre d'usagers qu'au travers d'un pourcentage. Ainsi dans le cas de la ville de Courbevoie qui travaille sur un projet de tableau de bord urbain, un accroissement de 100 usagers représente une évolution de $+0,1 \%$. Le chiffre brut est ainsi 
beaucoup plus adapté au repérage d'évolutions mineures... Or les évolutions seront probablement le plus souvent mineures puisque l'on se propose de publier cette information tous les mois.

2. Communiquer sur un nombre d'usagers est probablement plus efficace que sur un pourcentage. Cela rend la communication plus proche de l'humain. Ainsi, l'élu s'intéressera probablement davantage à un nombre d'usagers qu'à un pourcentage.

3. Les destinataires du tableau de bord - élus, services de la ville, associations locales de citoyens - connaissent la taille de la population, ils peuvent très facilement mettre en rapport les évolutions exprimées en chiffre brut avec celle-ci. La mention de la taille de la population en légende ou en commentaire peut toutefois compléter utilement ce graphique.

Ces chiffres peuvent être fournis en l'état (voir ci-dessus) ou rapportés à la population à desservir sous forme d'un pourcentage. Ils rendent compte de l'attractivité et de l'accessibilité des bibliothèques. Ils constituent une première série d'indicateurs permettant une surveillance du bon fonctionnement des bibliothèques et de leur niveau d'attractivité. En cela, ils sont adaptés à une fourniture relativement fréquente à l'élu référent. Un rythme mensuel semble intéressant. Analysés sur la durée, ces chiffres peuvent souligner le changement du positionnement des bibliothèques dans la population avec par exemple une moindre attractivité de la fonction de prêt et une plus grande attractivité de la fonction « lecture, étude sur place ». La ou le responsable de la bibliothèque peut être intéressé.e par des indicateurs introduisant une dimension qualitative à l'analyse des évolutions. Ainsi, certains de ces indicateurs (prêts, consultations à domicile des ressources numériques...) peuvent être déclinés par type de document. Cela aboutit toutefois à des données d'interprétation plus délicate car croisant des questionnements sur les politiques d'acquisition (attractivité de la bibliothèque) et sur l'accessibilité de ses services (en rapport avec les horaires d'ouverture, l'implantation de ses locaux, etc.).

Pour un approfondissement, une dimension qualitative peut également être introduite en reprenant chacun de ces indicateurs et en le déclinant par grands groupes d'usagers. Ces groupes d'usagers peuvent être définis par tranche d'âges, par quartier du domicile ou par catégorie socioprofessionnelle. Cette déclinaison permet de mieux cerner l'attractivité et 
l'accessibilité de la bibliothèque pour chacun de ces groupes. Mieux vaut toutefois disposer pour chaque groupe d'une quantification de la population totale faisant partie du groupe afin d'effectuer une analyse plus structurée. C'est en effet en rapprochant le nombre d'usagers appartenant à un groupe et la population du groupe que l'on pourra identifier une sous-représentation ou surreprésentation du groupe dans l'ensemble des usagers.

Ces indicateurs affinés - déclinaisons par type de document et par groupe d'usagers - peuvent alimenter une analyse plus structurée de l'attractivité et de l'accessibilité des bibliothèques. La multiplication des indicateurs qui en résulte conduit à les positionner à un deuxième niveau de diffusion. Ils sont davantage associés à une démarche d'évaluation que de surveillance.

\section{Thème 2 : rendre compte de la bonne exécution des missions et de l'investissement dans les grands domaines d'intervention}

Chaque bibliothèque doit recevoir de sa tutelle une formulation précise des missions qui lui sont confiées. Si cette tutelle s'inspire du Manifeste de l'Unesco, elle devrait formuler des missions en lien avec le droit à la culture, le droit à l'information et le droit à la formation. La charte du droit fondamental des citoyens à accéder à l'information et aux savoirs par les bibliothèques (Charte Bib'Lib de l'Association des bibliothécaires de France $)^{6}$ actualise le Manifeste de l'Unesco ; elle fournit une source complémentaire de réflexion sur les missions des bibliothèques dont il serait intéressant de rendre compte.

\section{Le droit à la culture}

Concernant le droit à la culture, les bibliothèques sont principalement positionnées dans une fonction de mise à disposition des œuvres culturelles. Plusieurs exigences sont associées à cette fonction, notamment l'accès libre et sans discrimination à toutes les cultures. Selon la Charte Bib'lib, " les collections, ressources et contenus disponibles dans ou par les bibliothèques reflètent la pluralité et la diversité de la société et doivent être exempts de toutes formes de censure idéologique, politique, religieuse ou

6. Charte Bib'Lib. [En ligne] : < http://www.abf.asso.fr/1/22/530/ABF/bib-lib-bibliotheque-pour-1acces-libre-a-l-information-et-aux-savoirs $>$. 
de pressions commerciales ». Comment donner à voir en quelques indicateurs cette pluralité et cette diversité des collections ? Est-ce que cela relève plutôt de l'expression formelle d'une politique d'acquisition ? N'est-il pas possible de quantifier les acquisitions ou les collections et de montrer que l'on reste bien dans le cadre défini par cette politique d'acquisition ? Ne serait-ce pas au bout du compte un des aspects essentiels du rapport d'activité annuel que de pouvoir montrer comment la bibliothèque a veillé à appliquer strictement la politique d'acquisition dont la mise en œuvre lui a été confiée ? En termes d'indicateurs, cela conduirait à fournir des volumes d'acquisition dans les différents domaines identifiés par la charte ou la politique d'acquisition. Ces volumes peuvent s'exprimer en chiffres bruts (nombre de documents acquis ou valeur d'achat de ces documents) ou être rapportés au budget total d'acquisition.

\section{Le droit à l'information : politique d'acquisition et vie locale}

Concernant le droit d'être informé, selon la Charte Bib'lib, il s'agit de permettre à chacun d'être en capacité de participer à l'innovation sociale et aux débats citoyens. Pour partie, rendre compte de l'activité de la bibliothèque dans ce secteur renvoie au paragraphe précédent et à la qualification des politiques d'acquisition dans les domaines particuliers de l'information, notamment la presse. Un autre pan d'activité des bibliothèques devrait peut-être faire l'objet d'un compte rendu spécifique : la mise à disposition des informations concernant la vie locale et plus spécifiquement celles de ces informations produites par la collectivité de rattachement. Les bibliothèques françaises sont assez peu positionnées sur cette dernière activité qui demeure de ce fait peu structurée, laissée à l'initiative de chaque service de la collectivité et au bout du compte très difficile d'accès pour le citoyen. Cela rejoint la réflexion sur le mouvement de libération des données publiques.

\section{Le droit à la formation}

Concernant le droit à la formation, rendre compte de l'activité de la bibliothèque dans ce domaine peut renvoyer à une qualification des politiques d'acquisition, à une quantification des actions de formation conduites par 
la bibliothèque, à une quantification de l'usage des ressources numériques d'autoformation... Outre la quantification des acquisitions identique aux aspects précédents, Il s'agirait donc de comptabiliser des usagers recourant aux actions de formation et d'autoformation et de quantifier des heures de formation et d'autoformation.

\section{Thème 3 : fournir des informations en temps réel, permettant d'améliorer l'expérience de l'utilisateur de la bibliothèque}

L'application mobile évoquée précédemment illustre parfaitement le type d'information que l'usager peut attendre de sa bibliothèque : nombre de places assises disponibles à un moment donné, nombre de postes Internet disponibles... La publication en temps réel de ce type d'indicateur est en soi un service rendu aux usagers, la compilation de cet indicateur à la semaine, au mois ou à l'année est intéressante pour qualifier de manière objective le niveau de saturation des équipements.

\section{Thème 4 : contribuer à la vie démocratique locale, en fournissant les informations nécessaires à l'instruction des décisions les plus structurantes concernant la bibliothèque}

La publication d'indicateurs permettant de qualifier l'activité de la bibliothèque dans ses différents champs d'intervention peut alimenter d'utiles débats sur l'emploi des moyens et sur les priorités d'intervention. Ainsi, il peut être intéressant au vu de ces indicateurs de s'interroger sur le niveau d'intervention dans le domaine du droit à la formation : la bibliothèque en fait-elle trop ou pas assez ? En fonction de la question spécifique que l'on se pose (par exemple l'accessibilité de la bibliothèque pour les personnes en situation de handicap ou la lutte contre la fracture numérique...), il faudra produire des indicateurs spécifiques essayant de quantifier tant les besoins (la population concernée) que les résultats (la part de cette population effectivement touchée par la bibliothèque). Construire ces indicateurs et le discours mettant en lumière les enjeux associés peut résulter d'une commande d'un élu qui souhaite étudier l'opportunité d'un recadrage de l'activité de la bibliothèque comme d'un choix de la bibliothèque. Dans un monde idéal, cela pourrait également résulter d'une commande 
de citoyens qui souhaiteraient voir la bibliothèque investir plus massivement certaines activités.

Réorienter la production de statistiques vers les questions essentielles en rapport avec le positionnement de la bibliothèque, son attractivité, les activités qu'elle doit développer... c'est tout de même plus gratifiant que produire la énième version d'un rapport statistique national qui n'intéresse que fort peu le citoyen ou l'élu de secteur. Pour mettre le tableau de bord au service de l'action et de la préservation des moyens de fonctionnement, il faut probablement se limiter à quelques indicateurs essentiels mais en les valorisant par une mise en forme attractive et un accompagnement de qualité. 


\section{2}

ÉVALUER L'IMPACT

DE SA BIBLIOTHĖQUE

SUR LE TERRITOIRE : MESURER

LA ZONE DE CHALANDISE OU DE RAYONNEMENT DE SA BIBLIOTHĖQUE

L'évaluation de l'impact de la bibliothèque sur le territoire et la population qu'elle est en mesure de desservir s'appuie bien souvent sur des indicateurs - relativement classiques - fournis par le système intégré de gestion de bibliothèque (SIGB) et par tout un faisceau d'éléments ressentis par le personnel. Par exemple, les membres d'une équipe peuvent avoir l'impression de toucher particulièrement un public d'hommes à la retraite car ceux-ci fréquentent très régulièrement et longuement les espaces de lecture des périodiques alors que cette catégorie d'usagers n'est pas la plus représentative des personnes composant le public de la bibliothèque lorsqu'on analyse les statistiques d'inscription, et n'est pas la plus forte composante de la population communale ou intercommunale.

Il nous faut donc sortir de la sphère du ressenti et des indicateurs classiques pour observer de façon plus attentive et efficace l'impact de la bibliothèque sur sa zone de chalandise, d'attraction ou de rayonnement, selon que l'on choisira les termes du marketing ou de l'électromagnétisme.

Cet examen permet dans un premier temps de dessiner un portrait de la population à desservir et ainsi de mieux connaître les publics potentiels de la bibliothèque pour, dans un deuxième temps, évaluer celle qui fréquente son établissement et esquisser le paysage des publics à consolider, à développer et surtout à fidéliser et conquérir !

\section{QU'EST-CE QU'UNE ZONE DE CHALANDISE OU DE RAYONNEMENT ?}

Avant de s'intéresser à la façon d'évaluer l'impact de la bibliothèque sur la zone de chalandise, il faut déjà définir ce concept qui semble à première 
vue relever plus du marketing et des pratiques commerciales voire de la géographie que de la bibliothéconomie.

Wikipedia nous renseigne sur la notion en nous indiquant que la "zone de chalandise d'un établissement commercial est sa zone géographique d'influence, d'où provient la majorité de la clientèle. Elle s'étend schématiquement sous la forme de courbes isochrones dont le centre est le point de vente, mais est en fait profondément modifiée à la marge par les implantations concurrentes et la sociologie des localités couvertes ${ }^{1}$. En reprenant les divers éléments décrits par l'encyclopédie collective et en les appliquant à la bibliothèque, on peut conclure que sa zone de chalandise correspond à la zone géographique où sont implantés ses publics potentiels.

La situation du territoire de la bibliothèque, tant sur le plan de la géographie que sur le plan de la démographie, de la composition sociale, des modes de transport..., entraîne évidemment de fortes variations de définition du périmètre de cette zone de chalandise.

Il faut d'emblée effectuer un distinguo entre les bibliothèques situées en zone urbaine et périurbaine et celles localisées en zone rurale ou semi-rurale.

En effet, un équipement situé en zone rurale ou semi-rurale a naturellement une zone de chalandise relativement large géographiquement mais certainement plus réduite en termes de population à desservir du fait d'une densité de population moyenne ou faible. A contrario, une structure urbaine voit sa zone de chalandise se réduire en matière de périmètre géographique mais se densifier en termes de desserte de la population. En outre, il faut dans les deux cas, tenir compte des relais que peuvent représenter les bibliothèques annexes, les points lecture ou bien les points de desserte des bibliobus gérés par la bibliothèque départementale dans le cas des bibliothèques rurales et des bibliothèques de quartier dans le cas des équipements urbains.

1. < https://fr.wikipedia.org/wiki/Zone_de_chalandise >. 


\section{COMMENT dÉFINIR LA ZONE DE CHALANDISE DE SA BIBLIOTHĖQUE?}

\section{Découpage Insee}

La démarche qui semble évidente est de s'appuyer sur les limites administratives de la commune ou de l'EPCI où se situe la bibliothèque.

On peut ainsi recourir à l'Insee qui propose pour les communes de plus de 10000 habitants et pour une partie des communes de 5000 à 10000 habitants le découpage infracommunal en îlots regroupés pour l'information statistique (IRIS) ${ }^{2}$. Ces IRIS définissent essentiellement des zones d'habitat comptant des populations de 1800 à 5000 individus. Le découpage en IRIS s'appuie sur les obstacles naturels (cours d'eau...) ou créés par l'homme (routes, voies ferrées...). L'Insee précise que « l'IRIS constitue la brique de base en matière de diffusion de données infracommunales. Il respecte des critères géographiques et démographiques et possède des contours identifiables sans ambiguïté et stables dans le temps ». L'Insee propose des fonds de cartes au format pdf présentant la commune découpée en quartiers selon l'IRIS. Les plans des quartiers sont également proposés par l'Insee avec identification des voies routières, ferrées et fluviales.

Toutefois, cette démarche évidente au premier regard n'est valable que pour les 1014 communes de plus de 10000 habitants et quelques villes " élues » de plus de 5000 habitants (1 166 communes).

Il nous faut donc élaborer une stratégie différente pour les bibliothèques des communes, aussi bien pour celles dont la population est inférieure à 5000 habitants (plus de 33000 communes du territoire !) que pour les autres dont les situations sont fort diverses.

\section{Découpage isométrique ou isochrone}

Ainsi, en absence de découpage proposé par l'Insee ou bien pour le compléter, le marketing géographique offre différents modes de définition d'une zone de chalandise. Wikipedia nous donne quelques éléments pour

2. < http://www.insee.fr/fr/bases-de-donnees/default.asp?page=infracommunal.htm >. Par exemple, pour le découpage IRIS de la Ville de Compiègne (Oise) : < http://www.insee.fr/fr/methodes/zonages/iris/cartes/carte_iris_60159.pdf >. 
la calculer sur lesquels il est possible de se baser pour définir celle de la bibliothèque.

Deux lignes directrices sont proposées :

- une zone de chalandise isométrique dont le calcul se base " sur la distance "directe" entre deux points, à vol d'oiseau ". Le calcul se base alors sur la distance maximale entre le public et la bibliothèque ;

- une zone de chalandise isochrone qui est basée « sur le temps nécessaire pour se rendre jusqu’à » la bibliothèque.

Ces modèles donnent une piste de départ pour définir la zone d'attraction de la bibliothèque. La zone de chalandise isochrone est certainement la plus adaptée. En effet, pour un usager le temps de déplacement pour se rendre à la bibliothèque est plus important que la distance en elle-même. On peut ainsi décliner la zone d'attraction de sa bibliothèque en traçant une série de cercles concentriques identifiant des temps de trajet et en évaluant l'impact sur la fréquentation de l'équipement.

Les temps et modes de trajet varient selon le contexte dans lequel s'inscrit l'équipement de lecture publique. En effet, les modes et les modalités de déplacement varient de façon conséquente en ville et à la campagne. Ainsi, en prenant pour base de calcul ce critère primordial du temps de déplacement, on peut dessiner et estimer la zone de chalandise de sa bibliothèque, en esquissant des cercles concentriques correspondant à la durée du trajet selon les modes de circulation doux ou rapides les plus adaptés à la situation de la bibliothèque.

On peut ainsi envisager trois zones permettant de définir cette zone d'attraction de la bibliothèque, selon que l'on mettra pour s'y rendre à pied, en vélo ou en voiture :

- de 5 à 10 minutes ;

- de 10 à 20 minutes ;

- plus de 20 minutes.

Ces différentes zones doivent être affinées et intégrer les habitudes de déplacement des publics éventuels de la bibliothèque. 
Les évaluateurs s’intéresseront particulièrement aux données et éléments suivants :

- les habitudes de déplacement des habitants du territoire en se reportant le cas échéant au schéma directeur des déplacements élaboré par la collectivité et les collectivités alentour dans le cas d'une zone d'influence touchant plusieurs communes ou intercommunalité que l'on trouvera auprès des services ad hoc de la collectivité ou à défaut auprès des directions régionales ou départementales en charge de l'aménagement du territoire. D’autres documents peuvent également fournir de précieuses informations comme les portraits de territoire, des dossiers d'étude commandés par les collectivités locales, départementales ou régionales ;

- seront ainsi estimés :

- la part des déplacements doux (à pied, en vélo...) ;

- la part des déplacements automobiles ;

- l'impact des moyens de transport en commun sur le territoire étudié et leur utilisation par la population concernée (notamment prendre en compte l'impact du coût des transports en commun ou de leur gratuité) ;

- les contraintes de stationnement pour tous les moyens de déplacement (voiture, vélo, trottinette... sans oublier les landaus et poussettes) : présence de parking à proximité de la bibliothèque, stationnement gratuit ou payant... ;

- la proximité de sites présentant un intérêt pour la population potentiellement usagère de la bibliothèque (lieux de travail, commerces, marchés, cinéma...) ;

- la présence d'obstacles naturels ou d'origine humaine ayant un impact sur les voies de communication et les modes de transport (fleuve, rivière, colline, route nationale, autoroute, voie ferrée...) ; 
- l'accessibilité de la bibliothèque aux personnes « empêchées »;

- tout autre élément propre au contexte géographique et urbain de la zone étudiée.

\section{Quels outils utiliser pour dessiner sa zone de chalandise ?}

Les cartes proposées par l'Insee ${ }^{3}$ sont une première ressource, toutefois réservée aux bibliothèques situées dans une zone découpée en IRIS. D'autres ressources sont disponibles mais demandent bien souvent des recherches au sein des différentes strates administratives des collectivités. Ainsi les bibliothèques peuvent orienter les recherches dans les directions suivantes :

- le schéma de cohérence territorial (SCoT) décrivant la zone dans laquelle est située la bibliothèque. Ce document d'urbanisme, élaboré à l'échelle d'un EPCI au minimum ou d'un groupement de communes, a pour but de mettre en cohérence les politiques d'aménagement du territoire dans les domaines de l'environnement, des paysages, de l'urbanisme, de la mobilité et des déplacements, de l'économie, du commerce, etc. ;

- les plan local d'urbanisme (PLU) et plan local d'urbanisme intercommunal (PLUi) ainsi que les cartes communales visant à organiser l'espace communal ou intercommunal ;

- la direction ou le service de politique de la ville dans les territoires où sont présents des quartiers « défavorisés » ou sensibles.

Ces directions ou services s'appuient sur des outils informatiques de traitement de l'information géographique - système d'information géographique (SIG) - permettant d'établir des cartographies du territoire axées sur certaines données. Certaines collectivités, des EPCI pour la plupart, rendent accessibles leur système d'information géographique 
et permettent la manipulation des données qu'elles ont libérées dans le cadre du mouvement d'open data 4 . À noter qu'on trouve également sur le Web des outils libres ou gratuits de géomarketing permettant de dessiner la carte de la zone de chalandise. Ces outils ne sont pas tous aisément manipulables, l'appui sur les services internes à la collectivité apparaît sûr et pérenne.

\section{QUELLES SOURCES EXPLOITER ?}

Une fois la zone de chalandise définie, la bibliothèque doit se poser la question des sources d'information et des ressources statistiques à exploiter pour connaître les caractéristiques des populations du territoire à desservir. Les statistiques fournies par l'Insee sont certainement la principale source d'information. Outre les données concernant la démographie communale ou infracommunale, l'Insee fournit un ensemble d'éléments sur la composition de la population tant du point de vue social, économique qu'en termes d'habitat. Ces données sont accessibles sur son site web ${ }^{5}$, à la rubrique base de données, sous-rubrique données locales. La bibliothèque trouvera les informations statistiques qui l'intéressent dans le résumé statistique et dans le dossier statistique complet ${ }^{6}$.

Ces informations peuvent être complétées par les résultats d'enquêtes initiées par les communes ou les intercommunalités. Ainsi, il faut mener l'enquête auprès des services travaillant sur le territoire comme la politique de la ville, le SCot, le PLU... afin d'obtenir des statistiques et des données réunies et analysées par l'Insee lui-même ou des cabinets d'étude spécialisés.

\section{QUELS INDICATEURS RETENIR POUR ÉVALUER LA ZONE DE CHALANDISE DE SA BIBLIOTHĖQUE?}

Une fois les sources d'information identifiées, il nous faut retenir des indicateurs pertinents. L'Insee nous fournit tout un éventail d'indicateurs

4. Voir notamment le site web de l'État consacré à l'open data : < https://www.data.gouv.fr/fr/ >

5. < www.insee.fr >.

6. Par exemple résumé statistique et dossier complet de la ville de Compiègne : < http://www. insee.fr/fr/themes/comparateur.asp?codgeo=COM-60159 >. 
dont certains retiennent notre attention et sont à mettre en perspective le cas échéant avec les statistiques obtenues par le SIGB équipant la bibliothèque (voir le tableau $\mathrm{n}^{\circ} 1$ ).

Les premiers indicateurs sont fournis par le résumé statistique de l'Insee. On retiendra les suivants :

- densité de la population ;

- nombre de ménages fiscaux ;

- part des ménages fiscaux imposés ;

- médiane du revenu disponible ;

- taux de pauvreté.

D'autres indicateurs sont fournis par le dossier complet des chiffres clés de l'Insee. On retiendra les suivants :

- lieu de résidence 1 an auparavant ;

- composition des familles ;

- lieu de travail des actifs de 15 ans ou plus ayant un emploi, qui résident dans la zone ;

- part des moyens de transport utilisés pour se rendre au travail ;

- catégories et types de logements ;

- ancienneté d'emménagement des ménages ;

- diplôme le plus élevé de la population non scolarisée de 15 ans ou plus.

Le tableau ci-après recense les données Insee à prendre en compte pour évaluer la zone d'attraction de sa bibliothèque et les met en correspondance avec les statistiques fournies par le SIGB. Les indicateurs sont répartis selon les chiffres clés de la nomenclature de l'Insee. 
TARLEAU 1 ÉVALUER LA ZONE DE CHALANDISE DE LA BIBLIOTHĖQUE : INDICATEURS À RETENIR

\begin{tabular}{|l|l|}
\hline \multicolumn{1}{|c|}{$\begin{array}{c}\text { Insee ou autre source de données } \\
\text { sur la zone de chalandise }\end{array}$} & \multicolumn{1}{|c|}{ SIGB de la bibliothèque } \\
\hline \multicolumn{1}{|c|}{ Chiffres clés Évolution et structure de la population } \\
\hline Population par tranche d'âge & $\begin{array}{l}\text { Inscrits par tranche d'âge, y compris les } \\
\text { renouvellements d'inscription et les pre- } \\
\text { mières inscriptions. } \\
\text { Prêts répartis par tranche d'âge et par } \\
\text { support (au minimum). } \\
\text { Prérequis : s'assurer que le SIGB est } \\
\text { en mesure de répartir les inscrits par } \\
\text { tranche d'âge et qu'il effectue des mises } \\
\text { à jour de cette répartition. }\end{array}$ \\
\hline Population par sexe & $\begin{array}{l}\text { Inscrits par sexe } \\
\text { Prérequis : s'assurer que le SIGB gère ces } \\
\text { données }\end{array}$ \\
\hline Population selon la CSP & $\begin{array}{l}\text { Inscrits par CSP ou PCS } \\
\text { Prêts par CSP ou PCS } \\
\text { Inscrits par catégorie, tarification... selon } \\
\text { les modalités de gestion de la bibliothèque } \\
\text { Prérequis : s'assurer que les CSP contenues } \\
\text { dans les tables du SIGB correspondent bien } \\
\text { à celles de l'Insee, s'assurer que le SIGB est } \\
\text { en capacité de gérer des données sur ces } \\
\text { éléments. }\end{array}$ \\
\hline Inscrits de la zone de chalandise \\
\hline
\end{tabular}

7. L'Insee distingue, depuis 2003, 8 groupes de professions et catégories socioprofessionnelles (PCS) : 1. Agriculteurs exploitants ; 2. Artisans, commerçants et chefs d'entreprise ; 3. Cadres et professions intellectuelles supérieures ; 4. Professions Intermédiaires ; 5. Employés ; 6. Ouvriers ; 7. Retraités et 8 . Autres personnes sans activité professionnelle. Ces 8 groupes sont découpés en 42 postes. 


\begin{tabular}{|l|l|}
\hline $\begin{array}{l}\text { Population par quartier } \\
\text { Données disponibles pour certains IRIS }\end{array}$ & $\begin{array}{l}\text { Inscrits par quartier } \\
\text { Prérequis : s'assurer que le SIGB permet } \\
\text { de gérer les quartiers. Lidéal étant de lier } \\
\text { la zone rue de la fiche adhérent avec le } \\
\text { fichier FANTOIR }{ }^{8} \text {. Les rues étant asso- } \\
\text { ciées à un quartier. }\end{array}$ \\
\hline \multicolumn{2}{|c|}{ Chiffres clés Emploi - Population active } \\
\hline $\begin{array}{l}\text { Population de 15 à 64 ans par type d'acti- } \\
\text { vité permettant notamment de faire res- } \\
\text { sortir la part des étudiants, des retraités } \\
\text { et des chômeurs dans la population. Ces } \\
\text { données peuvent être éventuellement } \\
\text { complétées par les ménages répartis } \\
\text { selon la CSP de la personne de référence. }\end{array}$ & $\begin{array}{l}\text { Inscrits par CSP ou PCS } \\
\text { Ins modalités de gestion de la biblio- } \\
\text { thèque }\end{array}$ \\
\hline \multicolumn{2}{|c|}{ Chiffres clés Diplômes - Formation } \\
\hline $\begin{array}{l}\text { Taux de scolarisation selon l'âge } \\
\text { Inscrits par niveau scolaire }\end{array}$ \\
$\begin{array}{l}\text { Inscrits par tranche d’âge } \\
\text { Prérequis : s'assurer que le SIGB gère au } \\
\text { minimum les grandes étapes scolaires : } \\
\text { maternelle, élémentaire, collège, lycée... }\end{array}$ \\
\hline
\end{tabular}

Comment exploiter les résultats, quels impacts sur la bibliothèque (politique documentaire, accueil...) ?

Après avoir défini les indicateurs à utiliser pour mesurer la zone de chalandise, il nous faut préciser leur exploitation pour mieux connaître la population à desservir et les impacts sur l'offre de services, le fonctionnement et l'organisation de la bibliothèque.

Le tableau ci-après met en relation l'indicateur retenu et son exploitation.

8. Le fichier FANTOIR (pour Fichier ANnuaire TOpographique Initialisé Réduit ; ancien fichier RIVOLI) répertorie les noms des voies et des lieux-dits de l'ensemble des communes françaises. Ce fichier est produit et mis à jour annuellement par la Direction générale des finances publiques : < https://www.data.gouv.fr/fr/datasets/fichier-fantoir-des-voies-et-lieux-dits/ >. 
TABLEAU 2 ÉVALUER LA ZONE DE CHALANDISE DE LA BIBLIOTHĖQUE : EXPLOITATION DES INDICATEURS

\begin{tabular}{|c|c|}
\hline $\begin{array}{c}\text { Indicateurs retenus } \\
\text { [chiffres de l'Insee et éventuellement } \\
\text { statistiques fournies par le SIGB] }\end{array}$ & $\begin{array}{c}\text { Quelques pistes pour exploiter } \\
\text { les indicateurs }\end{array}$ \\
\hline Densité de la population & $\begin{array}{l}\text { La densité de la population est un indica- } \\
\text { teur entrant dans l'évaluation des modes } \\
\text { de déplacement des publics. Une faible } \\
\text { densité indique en général une dissémi- } \\
\text { nation de la population et des déplace- } \\
\text { ments nécessitant un moyen de locomo- } \\
\text { tion motorisé qui peut être un frein à la } \\
\text { fréquentation de la bibliothèque. }\end{array}$ \\
\hline Nombre de ménages fiscaux & \multirow{4}{*}{$\begin{array}{l}\text { Ces indicateurs dressent le paysage de la } \\
\text { population à desservir et permettent d'iden- } \\
\text { tifier des publics plus enclins à utiliser une } \\
\text { offre culturelle (CSP+) et d'autres éloignés } \\
\text { de la sphère culturelle "légitime ". }\end{array}$} \\
\hline Part des ménages fiscaux imposés & \\
\hline Médiane du revenu disponible & \\
\hline Taux de pauvreté & \\
\hline $\begin{array}{l}\text { Population par tranche d'âge/Inscrits par } \\
\text { tranche d'âge (1 } 1^{\text {re }} \text { inscription et renouvel- } \\
\text { lement), prêts par tranche d'âge }\end{array}$ & \multirow{4}{*}{$\begin{array}{l}\text { La connaissance précise de la réparti- } \\
\text { tion de la population par tranche d'âge, } \\
\text { par sexe, par CSP et par quartier le cas } \\
\text { échéant, rapportée au nombre d'inscrits } \\
\text { par tranche d'âge permet d'évaluer et de } \\
\text { distinguer précisément les publics tou- } \\
\text { chés, fidélisés et à développer. De même, } \\
\text { le suivi des prêts par tranche d'âge et sup- } \\
\text { port permet d'adapter les collections aux } \\
\text { attentes des publics. Il faut donc suivre, } \\
\text { rapporté à la population à desservir et par } \\
\text { thématique : l'évolution du nombre d'ins- } \\
\text { crits actifs; l'évolution du nombre de nou- } \\
\text { veaux inscrits ; l'évolution du nombre de } \\
\text { réinscriptions ; l'évolution des prêts selon } \\
\text { les tranches d'âge et les supports prêtés. } \\
\text { Exemple: que met-on en œeuvre pour déve- } \\
\text { lopper les publics d'adolescents et de jeunes } \\
\text { adultes (15-29 ans), peu présents parmi les } \\
\text { inscrits de la bibliothèque et difficilement } \\
\text { fidélisables mais représentant une part } \\
\text { importante de la population du territoire? }\end{array}$} \\
\hline Population par sexe/Inscrits par sexe & \\
\hline $\begin{array}{l}\text { Population selon la CSP/Inscrits par } \\
\text { CSP ou PCS, inscrits par catégorie, } \\
\text { tarification... }\end{array}$ & \\
\hline $\begin{array}{l}\text { Population par quartier/Inscrits } \\
\text { par quartier }\end{array}$ & \\
\hline
\end{tabular}




\begin{tabular}{|c|c|}
\hline Lieu de résidence 1 an auparavant & $\begin{array}{l}\text { Cet indicateur donne essentiellement des } \\
\text { informations sur la composante étudiante } \\
\text { de la population à desservir. } \\
\text { L'évolution de cet indicateur permet } \\
\text { d'adapter l'offre de la bibliothèque en } \\
\text { direction de ce public. }\end{array}$ \\
\hline Composition des familles & $\begin{array}{l}\text { Le nombre d'enfants par famille, de fa- } \\
\text { milles monoparentales peut orienter des } \\
\text { actions sur la parentalité, des partena- } \\
\text { riats avec des associations... }\end{array}$ \\
\hline $\begin{array}{l}\text { Population de } 15 \text { à } 64 \text { ans par type d'acti- } \\
\text { vité/Inscrits par CSP, inscrits par catégo- } \\
\text { rie, tarification... }\end{array}$ & $\begin{array}{l}\text { Cet indicateur donne des informations } \\
\text { précieuses sur la part des étudiants, des } \\
\text { retraités et des chômeurs dans la popula- } \\
\text { tion à desservir et leur évolution. Il per- } \\
\text { met de connaître précisément la cible } \\
\text { de population desservie et de suivre son } \\
\text { évolution. }\end{array}$ \\
\hline $\begin{array}{l}\text { Lieu de travail des actifs de } 15 \text { ans ou } \\
\text { plus ayant un emploi, qui résident dans } \\
\text { la zone }\end{array}$ & $\begin{array}{l}\text { Cet indicateur oriente le choix des ho- } \\
\text { raires d'ouverture et des plages horaires } \\
\text { pour les actions et animations adaptées } \\
\text { aux rythmes de vie et aux contraintes des } \\
\text { populations de la zone de chalandise. }\end{array}$ \\
\hline $\begin{array}{l}\text { Part des moyens de transport utilisés } \\
\text { pour se rendre au travail }\end{array}$ & $\begin{array}{l}\text { L'évolution de cet indicateur peut expliquer } \\
\text { la désaffection de certaines cibles de public } \\
\text { ou la difficulté à les fidéliser et orienter } \\
\text { des choix d'aménagement : stationnements } \\
\text { pour les voitures, les deux-roues, boîtes de } \\
\text { retour à proximité de la gare... }\end{array}$ \\
\hline Ancienneté d'emménagement des ménages & $\begin{array}{l}\text { Cet indicateur est essentiel pour évaluer } \\
\text { le degré de fidélisation des populations à } \\
\text { desservir. }\end{array}$ \\
\hline $\begin{array}{l}\text { Taux de scolarisation selon l'âge/Inscrits } \\
\text { par niveau scolaire, inscrits par tranche } \\
\text { d'âge }\end{array}$ & \multirow{2}{*}{$\begin{array}{l}\text { Le suivi de ces indicateurs vise à orienter } \\
\text { la composition des collections physiques, } \\
\text { le choix des ressources numériques et } \\
\text { surtout les modalités d'accès à l'offre de } \\
\text { services. }\end{array}$} \\
\hline $\begin{array}{l}\text { Diplôme le plus élevé de la population } \\
\text { non scolarisée de } 15 \text { ans ou plus }\end{array}$ & \\
\hline
\end{tabular}


Assurer une veille constante sur sa zone de chalandise permet à la bibliothèque de réfléchir à l'évolution et à l'adaptation de son offre de services et de ses collections aux attentes et aux besoins de la population à desservir. Les propos ci-dessus mettent l'accent sur la richesse des apports des chiffres de l'Insee sans lesquels un suivi précis et constant ne pourrait se faire. S'informer pour bien connaître le territoire et la zone de chalandise passe également par une veille constante sur l'environnement culturel, social, économique, éducatif... en participant notamment à des réunions (réunions municipales, de quartier, associatives, thématiques...) visant à mieux connaître les attentes et les besoins de la population à desservir dans ces domaines. 


\section{3}

\section{MESURER LA VALEUR SOCIÉTALE DE LA BIBLIOTHĖQUE PUBLIQUE : DÉFINITIONS, CRITĖRES ET MÉTHODES ${ }^{1}$}

par

Frank

Huysmans

et Marjolein

Oomes

\section{DES INTRANTS ET EXTRANTS AUX OUTCOMES}

L'évaluation des outcomes (résultats/effets) des bibliothèques est en vogue. Cela n'a pas toujours été le cas. Si l'on regarde l'évolution des normes ISO utilisées en bibliométrie, qui ont été en grande partie définies par la communauté professionnelle, on s'aperçoit qu'on est progressivement passé des « intrants » (inputs) et « extrants » (outputs) aux " outcomes ». La première norme, ISO 2789 (première version en 1974) recommandait de rassembler des statistiques sur les indicateurs d'intrants et d'extrants à des fins de comparaison internationale et de classement. Les intrants désignaient les budgets, le personnel, la surface occupée, les collections, les achats ; les extrants faisaient référence au nombre d'usagers inscrits et de visiteurs, aux prêts, aux prêts entre bibliothèques (PEB) et aux différentes manifestations organisées.

La norme IS0 11620 (première version en 1998) a introduit des indicateurs relatifs aux performances des bibliothèques : des éléments tels que la part du public visé ayant été atteint, le coût des prêts, les délais de satisfaction des demandes de prêts entre bibliothèques, la proportion des titres recherchés disponibles dans la collection, etc. Alors que les indicateurs d'intrants et d'extrants évalués par les premières normes sont de simples calculs (des chiffres absolus), ces indicateurs de performance sont des chiffres relatifs dans la mesure où ils rapportent ces différentes mesures entre elles (pour créer des ratios). Sur la base de ces données facilement quantifiables, ils donnent une idée de la qualité des services que la bibliothèque offre à ses usagers.

1. Le titre original de cette contribution est : "Measuring the societal value of the public library: terminology, dimensions and methodology". Elle a été traduite de l'anglais par Anne Robatel. 


\section{DE NOUVEAUX BESOINS POUR LES BIBLIOTHĖQUES}

Dans l'évaluation des performances des bibliothèques, deux tendances sont apparues comme sources de malaise pour les professionnels. Premièrement, les indicateurs utilisés étaient pour l'essentiel adaptés aux performances des bibliothèques nationales, bibliothèques de recherche et bibliothèques universitaires. Ils étaient d'un intérêt limité pour évaluer les bibliothèques de lecture publique ou les bibliothèques scolaires, dont les performances obéissaient à des critères spécifiques qui n'étaient pas pris en compte. Les bibliothèques publiques en particulier offrent une palette de services qu'on trouve rarement dans d'autres types d'institutions, tels que des " cafés langue $»^{2}$, des ateliers de lecture à voix haute pour les enfants, des formations aux compétences numériques, des clubs de lecture, etc.

Deuxièmement, les indicateurs permettaient d'établir des classements utiles d'un point de vue management. S'appuyant sur les intrants et les extrants, ils rendaient cependant mal compte de l'impact des services sur les usagers - sur leurs compétences professionnelles, leurs résultats scolaires ou universitaires, le développement de leur créativité, l'enrichissement de leur culture ${ }^{3}$.

Parallèlement, les directrices et les directeurs de bibliothèques commençaient à subir une pression extérieure grandissante (émanant surtout de la part des responsables des budgets dans les conseils d'administration universitaires et les municipalités) les incitant à faire la preuve de l'intérêt des bibliothèques pour les usagers et les collectivités.

\section{PRENDRE EN COMPTE L'IMPACT DES BIBLIOTHĖQUES}

Depuis quelques années, une troisième norme est entrée en vigueur, ISO 16439 (première version en 2014), qui met au premier plan la question de l'impact des bibliothèques dans leur évaluation. Il ne s'agit plus seulement d'évaluer intrants et extrants mais aussi d'intégrer les outcomes au tableau

2. Ces « Cafés Language » constituent une offre fréquente dans les bibliothèques du Nord de l'Europe : ils permettent à des populations nouvellement arrivées de pratiquer la langue locale. Voir par exemple "Improve your Finnish in the library's language café". [En ligne] : < http://www.helmet.fi/en-US/Events_and_tips/News_flash/Improve_your_Finnish_in_the_librarys_lan(15035) >.

3. Pierre-Yves Renard, "ISO 2789 and ISO 11620: Short presentation of standards as reference documents in an assessment process", LIBER Quarterly, 2007, 17 (3/4). 
d'ensemble. Le tableau suivant tente de clarifier la distinction entre extrants et outcomes lorsque ces termes sont appliqués aux bibliothèques publiques.

\section{TABLEAU \\ COMPARAISON DES EXTRANTS ET OUTCOMES DES BIBLIOTHĖQUES PUBLIQUES}

\begin{tabular}{|c|c|c|c|}
\hline $\begin{array}{c}\text { Raconter } \\
\text { la bibliothèque }\end{array}$ & Extrant & & Outcome \\
\hline $\begin{array}{l}\text { Exprimé en missions } \\
\text { (ce que nous affi- } \\
\text { chons, } \\
\text { ce que nous } \\
\text { cherchons à faire) }\end{array}$ & $\begin{array}{l}\text { Produits/services/ } \\
\text { activités }\end{array}$ & $\begin{array}{l}\text { Distinction entre } \\
\text { " les gens qu'on } \\
\text { sert » et « les gens } \\
\text { qu'on atteint» }\end{array}$ & $\begin{array}{l}\text { Changement/effet } \\
\text { perceptible } \\
\text { (sur les comporte- } \\
\text { ments, les compé- } \\
\text { tences, les attitudes, } \\
\text { les situations de vie, } \\
\text { le statut) }\end{array}$ \\
\hline $\begin{array}{l}\text { (Choses que nous } \\
\text { disons) }\end{array}$ & $\begin{array}{l}\text { (Choses que nous } \\
\text { faisons et que nous } \\
\text { [devrions] mesurer) }\end{array}$ & & $\begin{array}{l}\text { (Choses que nous } \\
\text { voulons savoir) }\end{array}$ \\
\hline $\begin{array}{l}\text { Stimuler la lecture } \\
\text { et améliorer le } \\
\text { niveau de compé- } \\
\text { tences linguistiques }\end{array}$ & $\begin{array}{l}\text { Collection (quantité/ } \\
\text { qualité) ; accueil } \\
\text { de formations, } \\
\text { de groupes de dis- } \\
\text { cussion littéraires ; } \\
\text { prêt de livres et } \\
\text { de matériel pédago- } \\
\text { gique aux écoles }\end{array}$ & $\begin{array}{l}\text { Distinction entre } \\
\text { ressources utilisées } \\
\text { et usages de ces } \\
\text { ressources par les } \\
\text { participants }\end{array}$ & $\begin{array}{l}\text { Les gens ont-ils } \\
\text { vraiment lu les } \\
\text { livres qu'ils ont em- } \\
\text { pruntés? Ont-ils été } \\
\text { inspirés/surpris? } \\
\text { Leurs compétences } \\
\text { de lecture ont-elles } \\
\text { progressé ? Leur } \\
\text { horizon a-t-il été } \\
\text { élargi ? Liront-ils } \\
\text { plus souvent? }\end{array}$ \\
\hline $\begin{array}{l}\text { Encourager } \\
\text { l'engagement local } \\
\text { et la participation } \\
\text { citoyenne }\end{array}$ & $\begin{array}{l}\text { Organisation de } \\
\text { réunions, confé- } \\
\text { rences, débats. } \\
\text { Diffusion d'infor- } \\
\text { mations adminis- } \\
\text { tratives }\end{array}$ & $\begin{array}{l}\text { Distinction entre } \\
\text { participants, visi- } \\
\text { teurs, brochures } \\
\text { distribuées, statis- } \\
\text { tiques internet }\end{array}$ & $\begin{array}{l}\text { Les gens ont-ils } \\
\text { appris de nouvelles } \\
\text { choses? Ont-ils } \\
\text { rencontré de nou- } \\
\text { velles personnes? } \\
\text { Les informations } \\
\text { obtenues les ont- } \\
\text { elles fait changer } \\
\text { d'avis ? Ont-ils été } \\
\text { incités à s'engager } \\
\text { davantage dans la } \\
\text { vie citoyenne? }\end{array}$ \\
\hline
\end{tabular}

Source: Huysmans and Oomes, "Measuring the public library's societal value: A methodological research program”, IFLA Journal, 2013, 39 (2), p. 170. 
L'étude de la littérature récente sur le sujet permet de faire ressortir six types d'outcomes propres aux bibliothèques publiques.

ENCADRÉ

SIX TYPES D'OUTCOMES POUR LES BIBLIOTHĖQUES PUBLIQUES

1. L'acquisition de nouvelles connaissances et/ou des progrès de compréhension;

2. le développement de certaines compétences (par exemple, savoir candidater à un emploi ou acquérir des savoir-faire numériques) ;

3. un changement dans les attitudes, les normes et les valeurs sociales (par exemple à l'égard des personnes issues d'autres cultures, comme les demandeurs d'asile) ;

4. un effet sur les activités et les comportements ;

5. un changement de statut social et de conditions d'existence (lié à un nouvel emploi ou une meilleure situation financière) ;

6. des effets émotionnels ou intellectuels, la découverte de nouvelles expériences (le plaisir, linspiration, la créativité, la confiance en soi).

Bien sûr, l'évaluation ou la quantification de tels outcomes va bien au-delà des données que l'on peut facilement mesurer entre les murs d'une bibliothèque. Mais avant d'en venir aux questions méthodologiques, évoquons les enjeux conceptuels.

\section{IMPACT, VALEUR, OUTCOME : EFFORT DE CLARIFICATION TERMINOLOGIQUE}

Lorsque l'on parle de ce que les bibliothèques produisent, une quantité pléthorique de termes est employée. La littérature spécialisée parle des " effets », de " l'impact » (décliné parfois en impact individuel/impact social), des « bénéfices » (ou des bénéfices perçus), des "performances », de la « valeur », des " outcomes », du " retour sur investissement » (RSI ou ROI en anglais) et du " retour social sur investissement » (RSSI our SROI en anglais) de la bibliothèque. La norme ISO 16439 vise à éclaircir ces débats en définissant chacun de ces termes. Notre propre analyse de ces définitions fait apparaître six dimensions sous-jacentes à ce que propose 
la norme. Ce qu'on désigne comme " effet » des produits/services offerts par bibliothèque peut être :

- positif ou négatif ;

- délibéré ou accidentel ;

- immédiat ou à long terme ;

- préalablement défini ou non ;

- tangible ou intangible ;

- réel ou potentiel.

La notion la plus générale est celle d’impact, terme défini par ISO $16439^{4}$ comme " un changement individuel ou collectif provoqué par les relations avec les services offerts par la bibliothèque. » On peut ajouter ici que les autres critères évoqués ci-dessus sont indifférents pour mesurer l'impact, qui peut être aussi bien positif que négatif, délibéré qu'accidentel, etc.

Toujours d'après les définitions ISO, la valeur des services d'une bibliothèque peut être conçue comme un changement positif, indépendamment des autres critères.

La notion de bénéfice est un terme encore plus spécifique. Elle renvoie à un effet à la fois positif, délibéré et réel. Peu importe en revanche qu'il se manifeste dans l'immédiat ou à long terme, qu’il soit préalablement défini ou non, tangible ou intangible.

En revanche, un outcome renvoie à "l'effet immédiat et préalablement défini de l'extrant lié aux objectifs fixés par la programmation de la bibliothèque ", qui comprend également les outcomes " de l'institution ou de la communauté dont fait partie la bibliothèque $»^{5}$. La dernière partie de cette définition signifie que les effets doivent être positifs, délibérés et réels, en plus d'être immédiats et préalablement définis. En d'autres termes, les outcomes doivent être conçus comme une catégorie particulière des effets produits par les bibliothèques. Ils sont étroitement liés à des programmes spécifiques lancés par les bibliothèques (par exemple, un projet visant à

4. ISO 16439: Methods and procedures for assessing the impact of libraries, 2014, p. 4.

5. Ibid., p. 7. 
améliorer la littératie ou l'acquisition d'une seconde langue) - voire à ses missions - plutôt qu'à l'offre plus générale de services tels que le prêt de livres et la mise à disposition d'une salle pour la consultation des journaux, magazines et livres.

\section{LES CRITÈRES QUI FORGENT LA VALEUR DE LA BIBLIOTHĖQUE PUBLIQUE}

Dans les programmes de recherche sur les bibliothèques publiques que nous avons menés aux Pays-Bas, nous avons cherché à évaluer la valeur des services généraux fournis aux individus, aux collectivités et à la société dans son ensemble, ainsi que les outcomes plus spécifiques de programmes particuliers. Dans les textes portant sur ce sujet, qui comportent à la fois des analyses théoriques/normatives et empiriques, différents aspects individuels et sociétaux sont mis en avant par la bibliothèque pour préciser l'influence qu'elle pense exercer ou qu'elle cherche à acquérir afin de produire des changements positifs. À partir d'une revue de la littérature approfondie, on en distingue cinq grandes catégories :

- la dimension sociale : on considère ici tous les apports relevant du domaine des relations sociales, des liens tissés entre individus et des formes d'engagement collectif. Les textes parlent alors de "l'engagement dans le collectif », de "participation », de "l'identité du collectif », de " cohésion sociale », " d'inclusion sociale », de « capital social »;

- la dimension culturelle : la bibliothèque remplit une importante fonction culturelle liée aux activités artistiques (elle permet d'acquérir des connaissances et de diffuser des informations culturelles) mais aussi au domaine multiculturel (diffusion de connaissances au sujet d'une grande diversité de cultures) ;

- la dimension éducative : l'apport concerne ici des sujets bien connus des bibliothèques tels que l'éducation à tout âge, la littératie, l'éducation aux médias, etc. Les apports sont souvent liés à l'acquisition de nouveaux savoirs et compétences, mais la 
bibliothèque peut aussi influencer la manière dont les individus appréhendent l'institution scolaire, l'éducation et le développement personnel. En outre, l'accès à l'information et son traitement sont des éléments spécifiques qui doivent être inclus dans le volet éducatif étant donné le lien étroit qui relie la connaissance et l'information ;

- la dimension économique : l'apport est ici lié au développement des ressources financières des individus et des collectivités. Les bibliothèques peuvent non seulement contribuer à réduire la pauvreté en offrant un accès à des infrastructures et des sources d'information gratuites, mais elles peuvent également aider les gens à acquérir des compétences utiles pour réussir professionnellement ou pour gérer leurs ressources, de même qu'elles peuvent contribuer à faire croître l'économie d'un quartier ;

- la dimension affective : ce domaine comprend tout ce qui relève de l'état mental, affectif et physique des individus ainsi que des expériences telles que la relaxation, l'amusement, l'éveil émotionnel ou le sentiment de voir son horizon s'élargir grâce à de nouveaux apprentissages. Les apports sont ici d'ordre affectif et renvoient à la valeur intrinsèque de l'expérience que représente la fréquentation d'une bibliothèque. La santé, en tant qu'elle est nécessaire au bien-être physique, relève de cette rubrique. En outre, les apports fournis sur le plan social, culturel, éducatif et économique ont des répercussions affectives positives. Aussi la dimension affective est-elle vaste (voir le schéma ci-après). 


\section{SCHÉMA}

LA VALEUR DES BIBLIOTHÈQUES PUBLIQUES APPRÉHENDÉE À TRAVERS CINQ DIMENSIONS

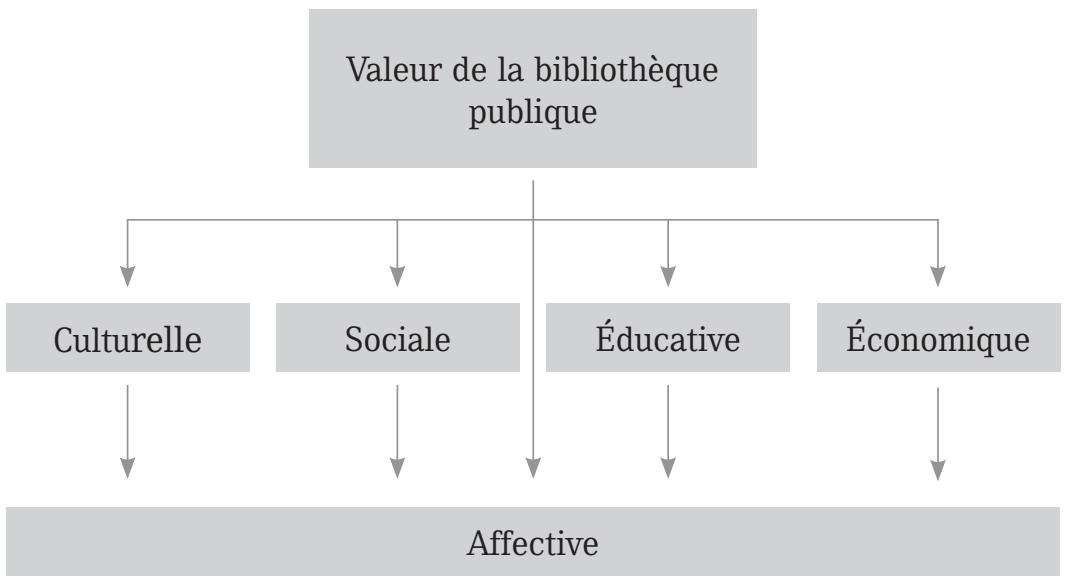

Sources : Huysmans Frank and Oomes Marjolein, "Measuring the public library's societal value: A methodological research program”, IFLA Journal, 2013, 39 (2), p. 174 ; Oomes Marjolein, Public library impact of individuals and society: A literature review (working title), Den Haag, Koninklijke Bibliotheek, à paraître.

\section{MESURER LA VALEUR SOCIÉTALE DES BIBLIOTHĖQUES PUBLIQUES}

Il est évidemment très compliqué de restituer les cinq dimensions de la valeur créée par une bibliothèque publique en tenant compte de tous les services qu'elle fournit.

L'élaboration d'une palette de critères multiples est une tâche de longue haleine dans laquelle nous sommes encore engagés. En lien avec ce projet, des études comparatives ont été lancées pour évaluer les performances du réseau des bibliothèques publiques néerlandais (du point de vue des bénéfices que les usagers estiment en retirer) par rapport à d'autres pays. Actuellement, la bibliothèque nationale des Pays-Bas développe un " programme de recherche sur les bibliothèques » ayant deux objectifs : 
1. évaluer l'utilité sociale du réseau des bibliothèques (publiques) à un niveau national en s'appuyant sur des critères locaux ;

2. aider les bibliothèques locales à améliorer leurs programmations, augmentant ainsi la valeur qu'elles représentent pour les individus et collectivités locales et par là même leur utilité sociale.

Dans ce programme, les animations particulières développées par les bibliothèques publiques (par exemple les actions visant à remédier à un faible niveau d'alphabétisme) sont pilotées de manière à ce qu'une bibliothèque locale puisse tirer profit des résultats pour améliorer ses activités. Parallèlement, il s'agit de faire apparaître une représentation au niveau national afin d'appréhender ce que l'ensemble des bibliothèques (environ 155 institutions comprenant environ 900 bibliothèques de quartier ou locales) réalise pour l'ensemble de la société.

L'idée principale est de faire circuler les informations du niveau national au niveau local et inversement :

- du niveau national au niveau local : développement de questionnaires normalisés intégrant des modules optionnels ;

- du niveau local au niveau national : utilisation de ces questionnaires dans les bibliothèques locales en collectant en complément des données qualitatives (interroger les usagers/participants) ;

- synthèse des données rassemblées au niveau local pour constituer une vue d'ensemble au niveau national.

Une autre question importante concerne la collecte des données. La norme ISO $16439^{6}$ énumère trois types de données :

- les données induites (à partir de l'évaluation des entrants et extrants, comme l'analyse des collections et des prêts) ;

- les données sollicitées (questions posées aux usagers, par exemple au sujet des outcomes réels/potentiels) ; 
- les données observées (observation systématique des faits, des actions et des comportements dans des situations pertinentes; tests).

Un prototype de procédure d'évaluation combinant ces trois types de données est actuellement en cours de développement aux Pays-Bas. Une fois terminé, il sera piloté et testé dans deux institutions composées de plusieurs bibliothèques locales (l'une a un ancrage urbain, l'autre rural).

Cette procédure sera modifiée puis à nouveau testée, éventuellement à plus grande échelle, en fonction des expériences menées et des points forts et des faiblesses qui en ressortiront. Au niveau national, les données concernant les services habituels offerts par les bibliothèques devraient être mises à disposition via une autre source, elle aussi en cours de développement : on y trouvera les collections des bibliothèques, les prêts, les usagers inscrits, les visiteurs, etc.

En rassemblant les données relevant à la fois des services habituels de la bibliothèque et des animations particulières, nous espérons mettre en place une base empirique solide qui démontrera l'utilité sociale des bibliothèques publiques. Nous invitons le lecteur à suivre les progrès de cette entreprise et à nous aider à mener à bien ce projet, car la coopération internationale et le partage des savoirs sont essentiels pour y parvenir. 


\section{4}

ARRÊTER, COMMENCER, CONTINUER :

Nathalie Clot

ÉVALUER POUR DÉCIDER

Less is more ${ }^{1}$

Lier étroitement « Pilotage et encadrement de la bibliothèque » et « Évaluation et conduite du changement » est un lieu commun des fiches de poste de direction en bibliothèque, notamment dans le référentiel Bibliofil ${ }^{2}$. L'ordre de présentation est pourtant révélateur : l'évaluation est toujours présentée dans un deuxième temps, comme une démarche a posteriori, venant après l'action, et non comme un élément d'aide au pilotage. L'évaluation y est aussi présentée comme un processus sui generis, tournée vers l'activité propre des bibliothèques, laissant peu de place à la connaissance de l'environnement et à ce qui compte sans forcément se mesurer. Résultat, dans bien des bibliothèques, l'évaluation se vide de son sens pour les équipes de terrain, et devient un objet technocratique, s'habillant de sigles variés. À l'université d'Angers, entre janvier et juin chaque année s'enchaînent l'enquête horaires du ministère, le rapport annuel de performances (RAP), sur la collecte des indicateurs du contrat quadriennal, l'enquête effectifs et fonctions (EEF), le contrat objectifs moyens (COM), l'ESGBU, puis le bilan d'activité pour le conseil documentaire et le rapport annuel qui assaisonnent, chacun à leur manière, les chiffres de l'année écoulée.

Que dit vraiment de nos organisations ce chapelet d'indicateurs ? À qui parlent-ils ? À la fois prisme imparfait de perception du réel et moyen d'appréhender de manière objective les activités, qu'apportent-ils aux décideurs, aux agents et aux utilisateurs pour les aider à prendre des décisions à court, moyen et long termes ? La première partie de cette

1. "Moins c'est mieux».

2. < http://www.enseignementsup-recherche.gouv.fr/cid23290/bibliofil-le-referentiel-de-la-filierebibliotheque.html >. 
contribution proposera des pistes de réflexion sur la relation entre indicateurs, coût humain de leur production et conduite du changement.

Nous verrons ensuite deux exemples concrets, mis en œuvre en 2015-2016 à la BU d'Angers. Ces deux changements, concernant les prêts et le site web, ont entraîné une baisse d'activité selon les indicateurs classiques et pourtant, permis de développer une approche qualitative, plus difficile à mesurer. Ces exemples illustrent la manière dont des indicateurs normatifs peuvent devenir des freins au changement et combien il est nécessaire de mettre la fabrique du chiffre au service d'une vision politique des services et de l'organisation de la bibliothèque.

Pour conclure, nous évoquerons comment les méthodes de design de l'expérience utilisateur - ou Design UX - peuvent nourrir au quotidien les projets d'une bibliothèque en se basant sur la connaissance des usages et l'observation active des utilisateurs.

\section{SAVOIR POUR DÉCIDER : MAÎTRISE OU ILLUSION ?}

\section{LA PETITE FABRIQUE DU CHIFFRE}

Collecter des indicateurs est devenu un impératif incontournable des organisations publiques et privées. Passage obligé des appels à projets, des conseils de gestion, des référentiels qualité, des contrats d'objectifs et de moyens, ils font l'objet d'une collecte organisée. Leur élaboration répond à des normes de plus en plus précises (voir supra).

Pour autant, peut-on évaluer le poids humain de cette fabrique du chiffre au sein des bibliothèques ? Que met chacun, en remplissant l'ESGBU, derrière les équivalents temps pleins (ETP) dits de pilotage et évaluation ? Combien d'agents relèvent-ils chaque matin des compteurs, reportent-ils des données dans des petits cahiers, puis compilent-ils au mois et à l'année ces données dans des tableurs ? Combien d'administrateurs de SIGB programment-ils en parallèle les requêtes SQL pour mieux connaître les usages des collections ? Combien pèse la passation d'une enquête Libqual et la mise en forme des résultats ? Combien de magasiniers parcourentils chaque année les rayonnages avec un mètre ruban pour donner une estimation précise du « nombre de mètres linéaires de périodiques en 
magasin » pour l'ESGBU, sans projet de réaménagement à la clé ? Combien d'administrateurs de documentation électronique sont-ils priés de compter leurs périodiques et leurs livres électroniques pour ERE et réalisent une cueillette annuelle de statistiques Counter qui pèsent bien peu face à des décisions budgétaires contraintes au moment des renouvellements de négociations?

Souvent centralisées dans les organigrammes, ces fonctions sont, de fait, diffuses dans une multitude de fiches de poste. Les données récentes de l'ESGBU n'étant pas disponibles mi-2016, procédons à une estimation : si on estime en moyenne à 1 ETP par établissement le poids de la petite fabrique du chiffre, c'est une entreprise de 80 ETP pour l'enseignement supérieur et sans doute autant pour les bibliothèques territoriales qui $\mathrm{y}$ sont consacrés, soit 240000 heures de travail par an. Quelle est la force de travail, en regard, dédiée à l'utilisation réelle de ces données, dont la collecte est souvent obligatoire, pour faire évoluer concrètement les processus de travail au quotidien, la manière d'organiser les espaces, les sites web, les services, le collectif de travail ? Au-delà de la collecte, combien de bibliothécaires utilisent-ils vraiment ces données pour agir?

\section{LE ROBIN DES BOIS EMPÊCHÉ}

$\mathrm{Au}$ niveau de l'allocation des moyens aux établissements, le constat est clair : toutes les petites universités pluridisciplinaires de province ont eu l'occasion de sourire à l'évocation de la dotation sur critères SYMPA ${ }^{3}$ (pour Système de répartition des moyens à la performance et à l'activité) qui soulignent des situations de « sous-dotation » et " sous-encadrement ». À l'université d'Angers, le déficit connu est d'environ 250 postes, partiellement compensés par le recours à l'emploi contractuel. Pourtant, en matière de pilotage national, il est politiquement intenable de prendre au riche Paul, théoriquement surdoté au vu des indicateurs, pour donner au pauvre Jacques. Les indicateurs SYMPA indiquent bien ce qu'il faudrait faire, mais les marges d'action ne sont pas là.

3. Financement des universités : Dominique Gillot et Philippe Adnot, "L'équité au service de la réussite de tous ", Rapport d'information $n^{\circ} 547$ (2012-2013). Rapport fait au nom de la commission de la culture, de l'éducation et de la communication et de la commission des finances du Sénat déposé le 24 avril 2013. [En ligne] : < http://www.senat.fr/rap/r12-547/r12-5471.pdf >. 
La clé de répartition du coût de la licence nationale Elsevier 2015-2018 est un autre bel exemple : l'approche par indicateurs a été adoucie par la realpolitik pour lisser les évolutions de coût pour les établissements. L'administration n'est pas Robin des bois, indicateurs ou non.

À l'échelle plus réduite d'un SCD ou d'un réseau de BM, combien de bibliothèques parviennent-elles vraiment à rééquilibrer le nombre de personnels entre sites pour suivre une évolution de l'activité ? Nous ne l'avons pas fait dans les BU d'Angers et il y a fort à parier que notre cas n'est pas isolé. Ces quelques exemples montrent que le retour sur investissement en termes d'action et de décision politique du coût de la collecte de chiffres n'est pas toujours évident.

\section{IL N'Y A PAS DE LUNETTES POUR VOIR TOUT NU OU LE PRISME IMPARFAIT DE PERCEPTION DU RÉEL}

Norbert Alter écrit, dans L'innovation ordinaire 4 : « les mesures de calcul de l'économie d'entreprise comme les instruments de gestion ne permettent pas aisément de connaître ce qui se passe dans la structure de travail. La réflexion est de ce point de vue limitée. Elle ne corrige jamais le déficit d'information du décideur. »

Attendre d'en savoir assez pour agir, vouloir des chiffres toujours plus précis est un paravent commode pour ne pas décider. Faire des approximations rapides lorsqu'une collecte grandeur réelle s'avère trop coûteuse ou aléatoire ou lorsque l'on réfléchit à quelque chose qui n'existe pas encore, est une compétence méconnue. Les estimations de Fermi ${ }^{5}$, du nom du grand physicien italien qui, au cours du projet Manhattan entre 1942 et 1945 a calculé de manière approximative toutes les charges nécessaires à la réaction en chaîne dans une bombe $\mathrm{H}$, sont souvent suffisantes pour orienter une décision, quitte à mesurer a posteriori son impact réel.

Décider en situation d'incertitude est un des moteurs principaux du changement. Dans un court volume au titre provocateur, Les stratégies absurdes : comment faire pire en croyant faire mieux, Maya Beauvallet,

4. Norbert Alter, L'innovation ordinaire, 6e éd., Paris, PUF, 2013 (coll. Quadrige), p. 185.

5. « Estimation de Fermi », Wikipédia, l'encyclopédie libre, 24 janvier 2016. [En ligne] : < http:// fr.wikipedia.org/w/index.php?title=Estimation_de_Fermi\&oldid=122680728 >. 
économiste, donne des exemples pratiques de stratégies politiques et managériales basées sur des indicateurs ayant mal tourné et en déduit que « les outils de pilotage ne remplaceront jamais le pilote. ${ }^{6}$ Les rapports d'activité $^{7}$ sont ainsi des outils de communication a posteriori, descriptifs, évoquant un état de stase. Ils sont rarement des leviers au service d'une dynamique au sein des bibliothèques.

Tous ceux qui ont essayé de s'appuyer sur les seuls indicateurs pour faire évoluer profils, missions et fiches de poste savent que les « difficultés surgissent lorsqu'on commence à croire que ces outils de reporting peuvent dispenser de manager et d'observer directement $»^{8}$.

Les chiffres et les résultats d'évaluation peuvent permettre d'objectiver des ressentis, mais ne sauraient suffire à convaincre un agent de faire évoluer une pratique dont bien des aspects ne sont pas mesurables. Combien d'introductions maladroites d'objectifs quantitatifs se sont soldées par des grèves du zèle, dans de nombreux secteurs où le respect de la lettre faisait perdre celui de l'esprit sous-jacent, lorsqu'ils ne créaient pas purement et simplement une défiance entre agents et responsables de bibliothèque, accusés de réduire un travail complexe à un ou deux indicateurs clés.

Maya Beauvallet cite Nate Furuta, président de Toyota qui dit qu' " une entreprise qui améliore ses indicateurs de résultats sans améliorer ses processus, ne vaut rien » et conclut qu' « il est toujours utile de laisser sa porte ouverte, d'aller voir ce qui se passe sur le terrain, de parler avec ses employés, ses utilisateurs ou ses voisins ", sans tomber dans les pièges que des mécanismes de mesure mal compris peuvent receler ni renoncer à l'usage de mesures bien tempérées.

L'homéostasie des organisations publiques fait que les bibliothèques et leurs agents ont été longtemps réfractaires à l'évaluation individuelle, se protégeant ainsi des effets pervers de l'indicateur roi mais perdant du

6. Maya Beauvallet, Les stratégies absurdes : comment faire pire en croyant faire mieux, Paris, Seuil, 2009 (coll. Sciences humaines).

7. Thomas Violet, Produire un rapport d'activité : Pourquoi ? Comment ?, Mémoire d'étude de conservateur des bibliothèques, sous la direction d'Isabelle Westeel : Villeurbanne, Enssib, 2016. [En ligne] : < http://www.enssib.fr/bibliotheque-numerique/documents/66455-produire-un-rapport-d-activite-pourquoi-comment.pdf $>$.

8. Maya Beauvallet, op. cit., p. 146. 
même coup une occasion de prise de recul, de dialogue et de réflexion partagée sur des pratiques en évolution constante.

\section{RENDRE L'INDICATEUR À L'USAGER}

Les paragraphes qui précèdent présentent une fabrique du chiffre orientée vers les tutelles, les cadres et les agents. Plusieurs outils, rendus possibles par la diffusion des objets connectés et le large accès aux réseaux, ouvrent d'intéressantes perspectives sur le partage en temps réel de certains indicateurs pour donner des marges de manœuvre et de décision aux usagers eux-mêmes.

Marc Maisonneuve ${ }^{9}$ évoque une petite application en ligne qui permet aux utilisateurs d'évaluer en direct le taux d'occupation de la bibliothèque pour décider d'y venir ou non et choisir le meilleur créneau horaire. Dans le même ordre d'idée, il est fréquent, dans des bibliothèques anglosaxonnes, de voir des collections organisées dans l'espace en fonction du taux de rotation des documents, et les services adaptés ainsi à la demande. Le lien entre évaluation et empowerment ${ }^{10}$ des politiques, des cadres, des agents et des usagers est possible : il n'est jamais facile de le mettre en œuvre et l'échelle macroscopique de bien des indicateurs les dissocie de l'échelon opérationnel s'ils ne font pas l'objet d'une élaboration et d'un usage délibéré et réfléchi pour aider à accompagner l'action de terrain. Deux exemples concrets, issus d'expériences récentes de la BU d'Angers, illustrent la manière dont nous avons pris des décisions pouvant paraître disruptives et avons tenté de vérifier a posteriori leur impact réel. Aux côtés des indicateurs normés, nécessaires à la comparaison d'efficience, il existe une vraie place pour un usage inventif des indicateurs de mesure : l'évolution à la hausse d'un ou plusieurs indicateurs classiques, comme le nombre de prêts ou de sessions sur un site web, ne saurait être l'unique horizon d'une approche qualitative des services.

9. Voir sa contribution : "Construire un tableau de bord pour la bibliothèque ? », p. 40.

10. Mécanisme par lequel les gens, les communautés, les organisations augmentent la maitrise de leur vie, selon la définition donnée par Julian Rappaport. 


\section{LESS IS MORE : QUAND SE LIBÉRER DE L'INDICATEUR TYPE PERMET DE CHANGER LE SERVICE}

\section{LES DROITS DE PRÊTS : MOINS DE PRÊTS, PLUS DE JOUISSANCE}

La BU d'Angers a modifié, en septembre 2015, les conditions d'emprunt de livres, inchangées depuis 2004. Les quotas de documents par utilisateur ont été supprimés, et une durée unique de prêt a été fixée à 3 mois pour un nombre illimité de livres. Le principe était de remplacer la règle de la bibliothèque par la règle de l'usager et de permettre à chaque utilisateur de rappeler à tout moment un document dont il aurait besoin.

Nous anticipions, en prenant cette décision, qu'elle aurait des répercussions sur trois indicateurs traditionnels, piliers des multiples rapports ESGBU, COM, RAP : le nombre de prêts, de prolongations et de réservations. L'évolution du nombre de prêts était tendanciellement à la baisse depuis 2010 et les réservations sous-utilisées, la période d'intérêt pour les manuels étant étroitement conditionnée à la durée des TD sur un sujet donné.

Mesurer quantitativement un volume de transactions sans tenir compte des conditions d'utilisation des collections nous paraissait peu satisfaisant. Nous avons donc cherché à mesurer quelque chose de plus qualitatif, qu'on pourrait qualifier de " confort d'utilisation des collections ». L'administratrice du SIGB à la BUA a eu une idée : produire un indicateur mettant en relation le nombre de prêts en cours avec la durée de chaque prêt, c'est-à-dire une donnée qui permet la comparaison avant/ après le changement des règles de prêt. 
TABLEAU 1

L'IMPACT DU CHANGEMENT DES RÈGLES D'EMPRUNT : NOMBRE DE PRÊTS VS DURÉE DE CIRCULATION

\begin{tabular}{|c|c|c|}
\hline & $\begin{array}{c}\text { Nombre de prêts } \\
\text { de documents }\end{array}$ & $\begin{array}{c}\text { Durée de prêt observée } \\
\text { en jours x nombre de prêts }\end{array}$ \\
\hline Février 2016 & 11141 & 590796 \\
\hline Février 2015 & 11471 & 316945 \\
\hline
\end{tabular}

Le volume des transactions de prêt est stable mais l'échantillon est trop court pour prouver quoi que ce soit de tendanciel.

L'amplitude d'utilisation des collections est accrue : l'indicateur de confort, c'est-à-dire le nombre de jours où un document est " aux mains de l'utilisateur » multiplié par le nombre de documents prêtés augmente de $86 \%$ entre février 2015 et février 2016 (590 796 jours de sortie en 2016, contre 316945 en 2015). Comment l'interpréter ? On peut émettre l'hypothèse que les nouvelles conditions de prêt améliorent le confort d'usage des collections en permettant de conserver plus longtemps les livres, et partant en limitant les déplacements et les prolongations de prêt. Là où on rappelait les livres pour les remettre en rayon dans la bibliothèque, nous les rappelons aujourd'hui pour les mettre à disposition d'un utilisateur qui en a besoin. Le nombre de réservations a doublé, augmentant le taux de rotation de certaines collections au moment où les gens en ont besoin.

Une autre manière de présenter ces données est particulièrement parlante : pour février 2015, l'usage cumulé des livres de la BUA par nos lecteurs représentait 868 années de jouissance. Avec la mise en place du prêt illimité et l'allongement de la durée de prêt à 3 mois, ce chiffre passe à 1818 années. On peut affirmer qu'en modifiant les règles d'emprunt, le droit de jouissance des collections, entendu comme " usage privatif temporaire d'un bien commun " est très largement accru. Sur les 6 premiers mois du nouveau dispositif, la jouissance des collections a doublé, passant de 5000 ans à 10000 ans. 
Cet exemple permet de montrer que plus n'est pas toujours synonyme de mieux et que la baisse ou la stabilité d'un indicateur classique peut cacher des effets vertueux.

Cette approche quantitative doit être complétée : une étude qualitative permettra de mesurer la perception des usagers qui ont bénéficié du service de rappel de livres ou au contraire l'ont subi, des non-emprunteurs pendant cette période, etc. Mesurer implique que nous sommes prêts à faire évoluer le service en fonction des résultats, liant de manière délibérée évaluation et action.

\section{UN SITE WEB RÉDUIT AUX FONCTIONNALITÉS ESSENTIELLES}

Résolus à limiter notre site web à ses fonctionnalités essentielles, nous avons choisi, à l'occasion d'une migration technique du site web de la BUA, d'immoler $95 \%$ des contenus rédactionnels du site, sur la base des indicateurs de consultation de pages l'année écoulée. Nos objectifs : limiter la production de contenus vite obsolètes et effectuer un audit régulier du site. Nous sommes en effet convaincus que la Longue Traîne, bonne pour Amazon, ne présentait pas le même intérêt pour un site de BU.

Nous étions conscients, comme dans le cas des prêts, que cela pouvait avoir un fort impact sur un indicateur clé de nos enquêtes préférées, le nombre de sessions.

Cette hypothèse s'est vérifiée : après refonte du site, le nombre de sessions a baissé. Creuser les indicateurs fournis par Google Analytics a été riche d'enseignements et a montré que sur le plan qualitatif, moins de contenu, c'est :

- moins de gens qui ne font qu'entrer/sortir du site (taux de rebond) ;

- plus de pages vues au cours d'une session;

- augmentation de la durée des sessions signifiant une meilleure " adhérence » au site. On parle en anglais de stickiness.

Il est trop tôt pour affirmer que Less is more. Cet exemple illustre néanmoins l'intérêt de ne pas se cantonner à un indicateur uniquement quantitatif. 


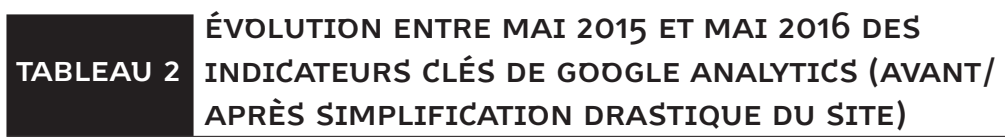

\begin{tabular}{|c|c|c|}
\hline & Mai 2015 & Mai 2016 \\
\hline Sessions & 43652 & 30841 \\
\hline Utilisateurs uniques & 24659 & 14363 \\
\hline Pages vues sur la période & 147747 (sur 1200 URL) & $\begin{array}{c}126973 \text { (pour 5 \% } \\
\text { contenu rédactionnel } \\
\text { migré) }\end{array}$ \\
\hline Pages vues par session & 3,3 & 4,1 \\
\hline $\begin{array}{c}\text { Durée moyenne session } \\
\text { Taux de rebond (sortie } \\
\text { immédiate du site) }\end{array}$ & 3 '49 $\%$ & $23 \%$ \\
\hline
\end{tabular}

Un usage créatif de la petite fabrique du chiffre est donc possible, appuyée sur des compétences en matière de requêtes sur les données brutes, via les SIGB et les outils de type Google Analytics.

Parler de créativité peut paraître surprenant dans une Boîte à outils sur l'évaluation : c'est pourtant une compétence indispensable, quoiqu'inattendue, tout comme celle de faire des estimations approximatives. Inventer et estimer sont en effet tout aussi nécessaires que bien connaître les normes, faire preuve de rigueur méthodologique et savoir rendre parlantes les données.

\section{PLAIDOYER POUR UNE DÉMARCHE DE DESIGN ORIENTÉ UTILISATEUR (DESIGN UX)}

Pour conclure, cette contribution ne peut qu'appeler à sortir des outils purement quantitatifs et normatifs pour amener l'ensemble des services 
d'une bibliothèque à adopter une approche globale de l'expérience utilisateur, nourrie d'observations de terrain, d'empathie et de recherches sur les usages réels de l'ensemble des services de la bibliothèque et les besoins fondamentaux des personnes qu'elles desservent.

Réserver des livres, aller à l'heure du conte ou être formé à la recherche documentaire ne sont pas les vrais besoins des gens. Formuler ainsi les problèmes revient à se centrer sur l'activité de la bibliothèque et du bibliothécaire. Mettre en œuvre une approche et des méthodes qui permettent de prendre conscience que l'utilisateur de nos services aspire à avoir de bonnes notes à ses examens, ou encore à trouver une occasion de partager un moment de qualité avec ses enfants ou travailler avec ses camarades, est un premier pas pour construire des services qualitatifs.

Concevoir une bibliothèque attentive à ses utilisateurs, c'est remplacer les choix fortuits, dictés par les habitudes ou le hasard, par des décisions délibérées. C'est ensuite évaluer si ces décisions répondent bien à l'objectif qui leur était fixé. Des allers-retours permanents entre action et évaluation permettent ainsi à une bibliothèque résiliente de se mettre en place et développent sa capacité à s'adapter et à s'améliorer en douceur au contact d'un environnement en constante évolution.

Là où la collecte d'indicateurs se révèle parfois un exercice solitaire, parfois ingrat, souvent peu suivi d'effets, les démarches de design orienté utilisateur (Design UX ${ }^{11}$ sont l'occasion de faire de l'évaluation un sport d'équipe. Elles permettent les échanges et d'associer observateurs et acteurs à la prise de décision. Les tests d'utilisabilité, bien décrits par Nicolas Alarcon ${ }^{12}$, sont une des méthodes pour améliorer les services en ligne d'une bibliothèque. Les cartes d'itinéraires ou parcours d'utilisateurs, recensant l'ensemble des points de contact entre la bibliothèque et ses utilisateurs sont aussi un outil de choix pour améliorer de manière globale un processus en s'attachant au diable caché dans les détails.

Promouvoir ces nouveaux savoir-faire, les diffuser dans les profils de poste, dans les organigrammes et accroître une culture de l'évaluation

11. Voir Aaron Schmidt et Amanda Etches, Utile, utilisable, désirable : redessiner les bibliothèques pour leurs utilisateurs, Villeurbanne, Presses de l'enssib, (coll. La Numérique), 2016. [En ligne] : < http://www.enssib.fr/presses/catalogue/utile-utilisable-desirable >.

12. Voir sa contribution : «Évaluer l'utilisabilité des sites web », p. 150. 
plus en prise avec le réel qu'avec les tableaux Excel est une option à considérer dans tous nos établissements. Humble, modeste, itérative, collective, une telle démarche ne peut que concourir à donner le pouvoir à chacun de prendre des décisions éclairées.

Car au final, évaluer sans décider est vide de sens.

Décider d'arrêter quelque chose, c'est arbitrer dans un univers où les ressources ne sont pas infinies et s'opposer de manière délibérée à l'entropie et à l'empilement des projets qui naissent sans apprendre à mourir. Arrêter, c'est aussi se donner l'opportunité de pouvoir commencer quelque chose d'autre en dégageant les moyens pour le faire.

Décider de continuer à faire quelque chose, en toute connaissance de cause, c'est se donner l'occasion de réaffirmer et conforter des choix. À chaque nœud de décision, une compréhension fine, régulière, objective et créative de la réalité aide à choisir et à justifier des décisions, sans jamais se substituer à l'imagination, au courage, au discernement, à l'honnêteté intellectuelle et, au final, au libre arbitre de tous les acteurs. 


\begin{tabular}{|l|l|}
\multicolumn{1}{|c|}{} & $\mathbf{5}$ \\
\hline ÉVALUER ET INSPIRER & par \\
LES TERRITOIRES DE DEMAIN & $\begin{array}{l}\text { Marie- } \\
\text { Christine } \\
\text { Jacquinet }\end{array}$ \\
\hline
\end{tabular}

De 2013 à 2015, les politiques d'action publique du département des Yvelines ont été profondément modifiées. Renouvellement de l'assemblée départementale, application de la loi NOTRe ${ }^{1}$, difficultés budgétaires, expression des besoins et urgences de la part des communes : une conjonction de facteurs a ainsi conduit les directions à revoir les composantes de leur démarche d'évaluation et à considérer désormais les territoires comme l'expression de la performance départementale.

La bibliothèque départementale, encadrée par un schéma de développement de la lecture arrivé à terme, a œuvré pendant cette période à analyser son leadership, ses processus de réalisation, l'impact de ses actions incluant la qualité de son organisation. Jusqu'alors, la politique de lecture publique était mesurée au regard de critères et d'indicateurs issus de son expertise métier : analyse des pratiques des bibliothèques (emprunts, inscrits, acquisitions, actions), de l'offre de service mise en perspective avec des moyennes nationales ou des normes de référence (heures d'ouverture, surfaces), des actions déployées (formations, actions culturelles).

Mais dans le nouveau contexte, la question posée était : quels sont, pour les territoires, les enjeux de développement de la lecture publique ? La performance de la politique de lecture s'est donc adossée à une démarche de développement territorial (diagnostic, objectifs, actions), prenant en compte les diversités identifiées et garantissant une cohésion harmonieuse, tout en exigeant l'introduction de nouvelles compétences dans l'organisation interne.

1. La loi du 7 août 2015 portant nouvelle organisation territoriale de la République. 


\section{DU DIAGNOSTIC DE LA LECTURE À UNE LOGIQUE DE TERRITOIRE}

Le contrat territoire lecture (CTL), signé en 2013 entre le département des Yvelines et l'État, a rapidement rendu incontournable la réalisation d'un diagnostic de la lecture publique. Des axes stratégiques ont donc été élaborés et proposés 6 mois après le début du contrat : 6 mois perçus comme une belle opportunité pour laisser reposer une partie des activités quotidiennes, pour réfléchir en équipes et groupes de travail à « quels sont les impacts des actions de la bibliothèque départementale ? ", à " comment faisons-nous, comment pourrions-nous faire, comment devrions-nous faire ? ». En clair, bénéficier d'une représentation actualisée des périmètres de l'intervention départementale pour la lecture.

Le cabinet conseil qui fut choisi pour accompagner cette phase déterminante s'est emparé d'une méthodologie éprouvée en la matière, associant les acteurs adéquats, collectant les données et les informations utiles. Entre autres, l'objectif était de dresser une carte précise de l'environnement de l'étude, et de recueillir toutes les informations relatives au territoire départemental (organisation territoriale, activités économiques et culturelles, composition sociodémographique, offre et demande en termes de formation, d'insertion, etc.). Hormis les contraintes de calendrier, puisque le diagnostic s'effectuait pour une bonne partie en été, plusieurs écueils furent rencontrés faisant affleurer à ce stade des pistes (largement exploitées par la suite), principalement au moment de l'état des lieux des besoins et des acteurs.

\section{MENER UN DIAGNOSTIC DE L'IMPACT : PREMIERS DÉFRICHAGES}

\section{Une mobilisation de sources documentaires hétérogènes}

Le recueil des données ne fut pas une tâche compliquée, tout service en étant producteur aujourd'hui, parfois même frénétiquement. La bibliothèque départementale collecte les bilans d'activité annuels de toutes les bibliothèques, et coordonne une campagne $\mathrm{SCRIB}^{2}$ qui rythme une bonne

2. Les BM sont invitées par les BDP à saisir leur rapport d'activité annuel sur SCRIB, outil proposé par le SLL. 
partie de ses activités. Les rapports publiés et les statistiques de toutes sortes produites par les directions du département, par les organismes ou les structures institutionnelles, privées ont été facilement identifiés et rassemblés. Mais comment croiser ces éléments pour une analyse pertinente ? Globalement, ils étaient le fruit d'outils hétérogènes, de référentiels propres, y compris au sein du département : par exemple, une même opération menée sur un territoire par la bibliothèque et la direction de l'action sociale bénéficiait de deux rapports d'évaluation, chacun prenant en compte un niveau d'impact territorial non concerté (souvent lié à une vision de politique sectorielle).

\section{une appréciation des réseaux d'acteurs juste amorcée}

La rencontre avec des opérateurs institutionnels, de terrain, des élus, des représentants de structures fut menée soit de façon bilatérale, soit sous forme de réunions territoriales, calées sur le découpage des territoires d'action sociale. Dans les réunions étaient conviés des bibliothécaires, mais aussi des acteurs culturels, sociaux et éducatifs. Si ces temps d'échanges ont permis d'impulser une dynamique transversale sur laquelle appuyer les nouvelles voies d'un développement de la lecture, ils ont mis en exergue l'impossibilité de représenter les relations actives entre les partenaires. Les réunions n'étaient pas suffisantes et aucun procédé sur le long terme n'était en place pour décoder les motivations des acteurs, leurs représentations des territoires, pour comprendre les divergences d'objectifs ou les conflits d'intérêts : bref, aucune possibilité d'analyse des logiques de partenariats.

Le diagnostic a certes contribué à des constats quant aux missions de la bibliothèque départementale. Mais, conséquemment, il a mis en évidence la nécessité de réviser les outils de mesure d'impact des actions liées à la lecture, d'inscrire les données des territoires comme matériau premier de toute analyse et d'envisager les objectifs du service dans une logique de développement territorial. 


\section{LA CONSTRUCTION D'UNE STRATÉGIE DE DÉVELOPPEMENT TERRITORIAL : TROIS ÉTAPES}

Si le diagnostic a été réalisé en quelques mois, il a fallu quatre fois plus de temps pour réfléchir, discuter, s'inspirer, tester et poser brique après brique le territoire comme point d'entrée des actions de la bibliothèque, puis ensuite redéfinir la politique de lecture publique à l'échelon départemental. Les indicateurs pertinents comme les performances clefs (internes et externes) ont été au cœur de chacune des étapes : une façon de s'assurer que les effets des actions sont mesurables, que les critères de réussite sont réalistes. Ainsi, la nouvelle stratégie de développement de la lecture publique mise en place depuis le $1^{\mathrm{er}}$ janvier 2016 s'est-elle fabriquée selon une progression en trois étapes, équivalentes à trois concepts décisifs.

\section{Étape 1 : comprendre les territoires d'action départementale}

Le mot « territoire » est une notion assez polysémique, presque magique, mais néanmoins significative des mutations institutionnelles et structurelles. Partant des capacités de ce terme à refondre l'action publique, et dans le prolongement de la réorganisation du département des Yvelines, le soutien aux bibliothèques est désormais décliné par territoires d'action départementale (TAD). Six territoires, six configurations différentes, six identités et six dynamiques pour exploiter leurs spécificités (spécificités naturelles, financières, humaines). Impossible dès lors d'aborder les bibliothèques avec la même offre de formation, le même soutien aux actions culturelles, la même offre de ressources. Comment concevoir un même programme d'actions sur un territoire rural dont $63 \%$ des communes ont moins de 1000 habitants, et dont $51 \%$ offrent une bibliothèque, face à un territoire urbain de 339715 habitants dont les 19 communes disposent toutes d'une bibliothèque ayant toutes intégré l'un des trois EPCI ? Chaque $\mathrm{TAD}$ inclut son système de référence, ses valeurs et ses enjeux, et oblige la bibliothèque à des actions différenciées, adaptées à la situation locale telle qu'elle est déterminée par le territoire lui-même. 


\section{Étape 2 : définir des actions globales liées à une politique de développement territorial}

Concevoir ses actions par le prisme du territoire pourrait s'avérer cloisonnant. Pour autant, si l'objectif de la politique de lecture publique est bien d'agir selon des spécificités, il est aussi de recomposer chaque territoire grâce à des actions plus globales, en modifier sa nature et son évolution pour le bénéfice des habitants. Les réseaux plurisectoriels sont alors des forces indispensables pour articuler ces dynamiques imbriquées. Par exemple, l'un des axes affichés du CTL fut un travail de longue haleine pour sensibiliser parents, médecins, bibliothécaires, libraires, professionnels de l'enfance à l'importance du livre pour le développement du langage. De ce fait, les indicateurs d'évaluation se sont dessinés en lien avec les autres politiques culturelle et sociale. À partir d'un questionnaire adressé à tous les partenaires, quel que soit le secteur lié, des outils d'analyse communs ont émergé : certains permettent de visualiser les caractéristiques des relations (juridiques, techniques, politiques, etc.), d'autres d'en apprécier la qualité (habitudes de travail, pérennité, concurrence, etc.), d'autres encore de mettre un indice de réalisation. Les politiques sectorielles s'articulent de cette manière à une politique de développement territorial partagée.

\section{Étape 3 : construire des projets de territoire}

La spécificité du territoire et les jeux d'acteurs impliqués ont contribué à redimensionner les projets de lecture publique. Entre l'ensemble des partenaires possibles sur un même territoire et les bibliothèques, il s'est avéré pertinent de développer la lecture publique en prenant appui sur tous les acteurs culturels. Ainsi, les activités de la bibliothèque départementale ont-elles été revisitées et modulées en projets de territoire. Ces projets passés par le filtre de la matrice SWOT ${ }^{3}$ sont déclinés en objectifs tactiques et opérationnels, chaque objectif est lui-même décliné en programme d'actions, incluant les résultats attendus et les moyens à engager.

3. Matrice permettant de réprésenter les forces et faiblesses, menaces et opportunités d'un projet SWOT ; en anglais pour Strengths (forces), Weaknesses (faiblesses), Opportunities (opportunités), Threats (menaces). 
Les activités de la bibliothèque s'exercent dorénavant à partir des projets de chaque TAD, tous secteurs culturels confondus.

\section{ENCADRÉ TROIS FICHES DIAGNOSTIC}

Les projets sont tous documentés de la même façon :

- une fiche territoire récapitulative des atouts et des faiblesses, des acteurs et des partenariats potentiels, des intentions en cours, des perspectives ;

- des fiches partenaires qui donnent à voir les capacités de l'environnement partenarial ;

- des fiches actions en faveur de tous les projets et reprenant le contexte, les objectifs, la description, les partenaires et les indicateurs de suivi.

Ces nouveaux outils forment la feuille de route des bibliothécaires, le « kit pratique » des rendez-vous avec les élus, les professionnels du terrain, et sont constamment mis à jour. Ils sont complétés par le rapport annuel destiné au ministère de la Culture et de la Communication. Utile pour représenter visuellement le taux d'équipement en bibliothèque (grâce aux cinq niveaux, selon la typologie en vigueur), ce rapport ne constitue en rien un outil de pilotage de politique publique, mais complète la boîte à outils des projets. Comment dès lors organiser la maintenance de ces instruments, leur ajustement voire leur évolution ? Comment gouverner le flux des mises à jour, réguler le suivi des avancées et garantir l'harmonisation des pratiques sur chacun des territoires?

\section{LE PILOTAGE AU CGEUR D'INTERACTIONS PROSPECTIVES}

Une cellule Pilotage Évaluation a été créée en 2016 sur la base d'une organisation opérationnelle simplifiée de deux personnes : un conservateur et un analyste programmeur. Les compétences du second sont clairement fléchées sur de la maîtrise de gestion de bases de données complexes, de transcription et d'ordonnancement des informations pour une analyse ultérieure, de conversion des calculs en cartes, schémas et autres représentations graphiques. Les compétences du premier recouvrent une 
expertise métier : il est question quotidiennement de concevoir et d'analyser, de coordonner, interpréter, comparer, mais aussi d'accompagner toute réalisation d'évaluation, en lien avec les autres directions du département, avec les autres instances régionales, nationales.

La feuille de route de cette cellule s'intègre absolument dans les phases avant et après projet :

- elle intervient dans la phase de diagnostic et fournit les analyses de manière à définir les besoins, échafauder un pronostic stratégique et détecter les moyens disponibles ;

- elle intervient dans la phase d'évaluation et applique les critères de réussite, met à jour les effets territoriaux et aide à calculer les impacts sociétaux.

Dans cette perspective, elle opère au milieu d'interactions internes et externes, signes de la vitalité constructive et créative de l'ensemble des acteurs, dans une dimension prospective et exploratrice.

\section{UNE RÉPARTITION DES RÔLES : LES ACTEURS INTERNES ET EXTERNES}

\section{Interactions internes}

Le tandem Pilotage Évaluation est au cœur d'un faisceau de circulations d'informations entrantes et sortantes. S'il fallait dessiner les synergies en présence, un atome et les électrons gravitant autour seraient parfaits. Un aperçu panoramique de quelques acteurs au cœur du processus :

- les chefs de projets : référents sur les territoires, transmettent les informations clefs, complètent des tableaux de bord et utilisent tout document produit par la cellule ;

- les experts ingénierie : utilisent les diagnostics pour bâtir programmes et plans (de formations, par exemple), et contribuent à la saisie des données au fil des opérations d'accompagnement ; 
- les autres directions : ont accès aux référentiels, aux supports constitués et apportent leurs propres données sur un espace de travail mutualisé dynamique ;

- les élus départementaux : sont destinataires des visuels et représentations graphiques de tout état des lieux chiffré.

Au regard de la répartition des rôles dans l'organisation interne, un travail important de référentiel commun a débuté, et est toujours en cours.

Se mettre d'accord sur les vocabulaires employés, sur les critères justes, sur les types de résultats atteignables et sur les impacts attendus pour une politique de lecture publique, c'est à coup sûr, en background, identifier des valeurs et les mettre en adéquation avec les autres politiques sectorielles.

\section{Interactions externes}

Dès les premières réunions partenariales dans le cadre du CTL en 2013, force a été de constater la méconnaissance des territoires chez les bibliothécaires communaux et intercommunaux. La cellule Pilotage Évaluation est apparue très vite comme un service support pour toutes les bibliothèques yvelinoises, ainsi que pour les acteurs culturels. Chaque établissement effectue généralement un diagnostic de sa zone de rayonnement, avec ses propres outils, ses moyens, dans une vision le plus souvent micro. Désormais, tous bénéficient des fiches diagnostic réalisées par le service, des fiches partenaires susceptibles d'être associées à des projets, tout en coopérant pour la collecte des données utiles : bref, la cellule agit comme un bureau d'ingénierie, en capacité de tracer des hypothèses de développement pour demain.

Des entreprises engagées dans des opérations de restructuration d'équipements ou d'aménagement d'infrastructures sont preneuses de résultats ordonnés et de cartes représentatives des forces en présence. La SNCF s'est servie d'une carte d'implantation et de circulation entre bibliothèques dans les zones rurales du département pour examiner la possibilité de convertir d'anciennes gares en espaces publics intégrant un service de prêt de livres. La Poste a utilisé les mêmes ressources dans le cadre de l'intégration de bureaux de poste dans les petites communes : la présence 
d'une bibliothèque, le déplacement des habitants pour emprunter, pour rendre les documents, la configuration sociale des emprunteurs... autant d'éléments infirmant ou confirmant une possible localisation d'un service postal en bibliothèque.

Cette démarche d'évaluation appliquée aux politiques de développement de la lecture dans les Yvelines est toujours en mode " fabrication », connaît des périodes d'expérimentation, et n'en est pas à ses derniers ajustements. La ligne de force qui la domine s'articule autour de deux aspirations : celle de redonner du sens et de la valeur à une politique publique de plus en plus considérée comme un soft power ${ }^{4}$, et pour cela, la confronter à des politiques plus larges, en stimulant toutes les coopérations accessibles ; et celle de mobiliser les indicateurs appropriés à une vision prospective, pour servir au mieux les métamorphoses territoriales en chantier.

4. Le soft power (puissance douce en français) est un concept issu des relations internationales. Il s'oppose à la force et la coercition, et désigne toute tentative d'influer sur les comportements à force d'influence et de persuasion. 
6

MESURER LA FRÉQUENTATION

DE 121 ÉTABLISSEMENTS :

par

Cécile

L'EXEMPLE DE LA BIBLIOTHĖQUE

Avallone DÉPARTEMENTALE DU VAL D'OISE

Les indicateurs habituellement recueillis en bibliothèque sont principalement liés à l'activité de prêt (nombre d'inscrits, nombre de prêts, collections, etc.). Ces chiffres pourtant ne font pas état du rôle social que jouent ces établissements publics dans la vie de la cité. En effet, nombreux sont les habitants qui fréquentent les bibliothèques et utilisent leurs services, voire participent à des événements, sans avoir envie ou besoin de s'y inscrire pour emprunter des ouvrages. Mesurer la fréquentation est alors une donnée essentielle pour avoir une vision réelle de l'activité de la bibliothèque.

À cet effet, la bibliothèque départementale du Val d'Oise a réalisé une étude quantitative du nombre d'entrées dans les 121 bibliothèques publiques du département.

\section{COMPTER QUOI ET COMMENT ?}

Le comptage s'est effectué à l'échelle départementale :

- sur une semaine complète, du lundi au dimanche ;

- sur les temps d'ouverture au grand public (hors temps d'accueil dédiés à des publics spécifiques).

Est comptée toute personne qui passe la porte de la bibliothèque, quel que soit son usage du lieu : prêt, lecture, attente, toilettes... 


\section{ENCADRÉ}

MESURER LA FRÉQUENTATION : LES CINQ ÉTAPES DE LA MISE EN ÆEUVRE

1. Monter une campagne de communication en amont pour sensibiliser les communes, les bibliothèques et leur public à la question de la fréquentation ;

2. Mettre en place plusieurs systèmes de comptage :

- le compteur automatique sur portique avec logiciel d'exploitation ;

- le comptage avec ticket à l'entrée ;

- le comptage manuel.

3. Prévoir un tableau de comptage qui doit permettre de dépouiller :

Pour le département du Val d'Oise, nous avons demandé aux bibliothèques de compter :

- les personnes fréquentant par tranche - horaire d'ouverture par jour ;

- la fréquentation découpée en matin et après-midi (exemple : 9 h 30 - 12 h 30 $=$ matin et $12 \mathrm{~h} 30-18 \mathrm{~h}=$ après-midi pour journée continue).

Cela nécessite donc de communiquer en amont ces éléments de collecte de l'information aux partenaires pour qu'ils puissent compter de manière différenciée.

Il faut surtout ensuite exploiter ces éléments et en rendre compte aux partenaires.

4. Prévoir un tableau de recensement global des résultats :

- recensement de la fréquentation des bibliothèques du département ;

- recensement des bibliothèques qui n'ont pas compté ;

- recensement de la fréquentation par jour ;

- calcul de l'extrapolation.

\section{Calculer l'extrapolation :}

Une fois les résultats collectés, une extrapolation a été faite sur 46 semaines pour obtenir un chiffre annuel (les bibliothèques du Val d'Oise étant en moyenne fermées 6 semaines par an).

Pour les bibliothèques qui n'ont pas compté, l'extrapolation est faite à partir de la surface de l'équipement :

Formule :

surface totale des bibliothèques $\mathrm{X}$ total de la fréquentation des bibliothèques qui ont compté

total de la surface des bibliothèques qui ont compté.

Pour le département, cette donnée est en effet la plus corrélée à la fréquentation, comme l'a démontré l'enquête réalisée en 2009 " Les facteurs de réussite des bibliothèques et des médiathèques publiques ». Cette extrapolation neutralise l'absence de chiffres pour ces quelques bibliothèques dans le total départemental. 


\section{QUELS RÉSULTATS POUR LE VAL D'OISE?}

$++++++++++++++++++++++++++++++++++++++++++++++++++++++++++++++++$

L'ensemble des bibliothèques publiques du Val d'Oise accueillaient en 2014 2,6 millions de personnes par an. Cette fréquentation est en augmentation de 34 \% par rapport à l'enquête réalisée en 2009 qui donnait un total de 2 millions d'entrées dans les bibliothèques, soit autant que le Parc Astérix. À titre de comparaison, la Cité des sciences en 2012 accueillait 2,6 millions de visiteurs ${ }^{1}$.

La moyenne départementale se situe à 25019 entrées par an et par bibliothèque. Afin de comparer les bibliothèques entre elles, il est possible de rapporter la fréquentation au nombre d'heures d'ouverture.

Selon les bibliothèques, la fréquentation varie de 1 à 210 personnes par heure d'ouverture, avec une moyenne de 34 personnes par heure d'ouverture. Mais la moitié d'entre elles accueillent moins de 9 personnes par heure.

1. < http://www.insee.fr/fr/themes/tableau.asp?reg_id=0\&ref_id=NATTEF05466 >. 


\section{PARTIE III}

\section{PILOTAGE}

1. ÉVALUER LES FORMATIONS À LA MAÎTRISE DE L'INFORMATION À L'UNIVERSITÉ : CONDITIONS, ENJEUX ET PERSPECTIVES par Hélène Coste

$++++++++++++++++++++++++++++++++++++++++++++++++++++++++++++++++$

2. LE SERVICE PUBLIC POSTÉ EN BIBLIOTHÈQUE : COMMENT MESURER L'EFFICACITÉ D'UN SERVICE ORDINAIRE ET DISCRET ? L'EXPÉRIMENTATION DE LA MÉTHODE AMÉRICAINE READ À LA BPI par Muriel Amar et Guilaine Thuillart

$+++++++++++++++++++++++++++++++++++++++++++++++++++++++++++++$

3. ÉVALUER LES ATTENTES ET PERCEPTIONS : L'OUTIL LIBQUAL +®, SES AVANTAGES ET SES LIMITES

par Frédéric Brodkom

$++++++++++++++++++++++++++++++++++++++++++++++++++++++++++++++$

4. ÉVALUER LA CONSULTATION SUR PLACE ET L'USAGE DES ESPACES :

LE CAS DE LA BIBLIOTHĖQUE DE SCIENCES PO PARIS par Cécile Touitou

$+++++++++++++++++++++++++++++++++++++++++++++++++++++++++++++++$

5. LE TRI PAR CARTES POUR AMÉLIORER L'ARBORESCENCE DU SITE WEB DE LA BIBLIOTHĖQUE DE SCIENCES PO PARIS par Joris Paillaré

6. ÉVALUER L'USAGE DES RESSOURCES NUMÉRIQUES : QUELS ENJEUX, QUELLES SOLUTIONS?

par Thomas Jouneau et Thomas Porquet

$+++++++++++++++++++++++++++++++++++++++++++++++++++++++++++++++$

7. ÉVALUER L'UTILISABILITÉ DES SITES WEB

par Nicolas Alarcon 
1

ÉVALUER LES FORMATIONS À LA MAÎTRISE DE L'INFORMATION À L'UNIVERSITÉ : CONDITIONS, ENJEUX ET PERSPECTIVES

La formation des étudiants à la maîtrise de l'information est aujourd'hui une réalité quotidienne de l'activité des BU. Si les bibliothécaires sont convaincus de la pertinence et de l'utilité de cet aspect de leur mission ${ }^{1}$ dans la réussite des étudiants, peu de données chiffrées viennent corroborer cette conviction. L'évaluation des formations à l'information et de leur impact sur la réussite étudiante reste un champ relativement peu défriché en France et de toute façon à chaque fois circonscrit à un établissement. De fait, c'est une activité difficile à évaluer par rapport à d'autres qui donnent lieu à des transactions informatiques ou à des mesures automatiques (on pense évidemment aux prêts et aux entrées). Or pour ce qui est des formations, un grand nombre de paramètres entre en compte puisqu'il s'agit avant tout d'une interaction humaine. Et quand bien même l'on parvient à comptabiliser ces actions, il reste plusieurs marches à gravir pour passer de l'évaluation quantitative à l'évaluation qualitative, et enfin à l'évaluation de l'impact des formations sur la réussite des étudiants, enjeu pourtant crucial.

\section{LES TROIS ÉTAPES DE L'ÉVALUATION}

\section{LA PREMIÈRE ÉTAPE : L'ÉVALUATION QUANTITATIVE}

Comme dans toute démarche d'évaluation, on commencera par collecter les statistiques d'activité, en veillant bien à recueillir l'ensemble des paramètres de chaque action de formation :

1. Pour exemple, dans un établissement comme la BU du Havre, sur 38 personnels de toutes catégories, 18 sont amenés à faire des formations, 8 de façon occasionnelle - moins de 5 heures par année universitaire - 10 de façon régulière. 
- le nombre d'heures dispensées/le nombre d'heures reçues par étudiant ${ }^{2}$;

- le nombre de personnes formées ;

- le nombre de formateurs.

Ensuite, il s'agit de caractériser ces formations :

- caractériser le public : selon le niveau, le cursus suivi ;

- caractériser les formateurs (qui peuvent être des bibliothécaires, des moniteurs étudiants, des enseignants, des professeurs de documentation);

- caractériser enfin le contenu de l'action de formation : de la simple visite des espaces à une formation complète en plusieurs modules.

\section{L'enquête annuelle des BU}

Les BU sont guidées dans cette phase de recueil des données par l'ESGBU qui demande chaque année de comptabiliser le nombre total d'heures dispensées, le nombre d'usagers concernés (avec la répartition par niveau), et la distinction hors ou dans les cursus (sur laquelle on reviendra). La recension de l'ESGBU correspond à l'année civile et donc potentiellement à l'effectif étudiant de deux années universitaires. Or si l'on veut calculer un indicateur important comme le ratio d'étudiants formés par rapport au nombre total d'étudiants inscrits à l'université, il faut prendre les chiffres de l'année universitaire pour rendre compte de la réalité des formations puisque le dénominateur commun est le chiffre des inscriptions sur l'année universitaire. Ce dernier est d'ailleurs suivi annuellement à l'université du Havre dans le cadre du contrat quinquennal d'objectifs qui lie l'établissement au ministère de l’Enseignement supérieur.

2. Un des problèmes principaux de la formation en licence est l'effectif : pour former 300 étudiants il va falloir 20 groupes de 15 étudiants, à raison de 2 heures par groupe, cela représente 40 heures. En termes de logistique et de ressources humaines, c'est souvent difficilement absorbable par les bibliothèques. 


\section{Les indicateurs du contrat quinquennal}

Ce contrat fixe les objectifs de l'université en matière de formation et de recherche. Une partie de la dotation annuelle est calculée en fonction des projets listés dans le contrat. Dans l'axe 2 « Renforcer l'offre de formation en cohérence avec l'identité scientifique », la sous-partie "Optimiser et moderniser la formation en termes de qualité » détaille un certain nombre d'actions concernant la BU et notamment « Renforcer l'accompagnement documentaire (formation, médiation) et participer aux démarches d'innovation pédagogique ", et " Proposer aux doctorants, dès leur inscription en thèse, un accompagnement documentaire personnalisé ainsi que des formations [...] en complément des activités de l'école doctorale. » Ces actions s'accompagnent d'un volet d'indicateurs. Ainsi l'indicateur spécifique 1 (IS 1) - « 0ptimiser la démarche qualité pour l'accompagnement à la réussite » - est créé " pour suivre et évaluer le dispositif d'accompagnement des étudiants, priorisé dans le projet stratégique d'établissement ». Sont comptabilisés les formations documentaires, les certifications C2I (pour Certificat Informatique et Internet) ${ }^{3}$ et CLES (pour Certificat de compétences en langues de l'Enseignement supérieur) ainsi que les PPP (pour Projet personnel et professionnel de l'étudiant). La bibliothèque s'est donc fixé une cible à atteindre - ratio d'étudiants formés - en fonction d'objectifs précisément définis. Chaque année nous devons mettre à jour l'indicateur et éventuellement la cible, en fonction de l'évolution du contexte qui a fortement changé pendant les quatre années du contrat (2012-2016). Nous avons décidé de détailler dans l'indicateur global les différents types de prestations :

- formations dans les cursus incluses dans les maquettes d'enseignement et généralement dispensées par des enseignants des disciplines ou des professeurs de documentation ;

- formations hors cursus proposées aux enseignants ou demandées aux bibliothécaires à l'occasion d'un travail précis à alimenter (rédaction d'une bibliographie, recherches pour un mémoire, etc.), généralement en Licence 3 ou Master.

3. Cette certification nationale valide des compétences en lien avec l'informatique et l'environnement numérique. 
La quantification permet également de répartir le nombre de personnes touchées par une action de formation suivant cette typologie cursus/hors cursus/ateliers (qui correspondent aux formations libres d'une heure à deux heures maximum dispensées sur des outils et thématiques diversifiés ${ }^{4}$ et qui peuvent aussi bien attirer le public étudiant que des doctorants, des enseignants ou même des personnels ou des publics extérieurs). Cette évaluation quantitative nous a permis de prendre conscience que nous touchions un public chaque année plus nombreux de lycéens, notamment grâce aux dispositifs destinés à faciliter la transition lycée-université, tels que "les cordées de la réussite » ou " un enseignant, un chercheur, une classe », mais aussi aux Journées portes ouvertes de l'université pendant lesquelles nous accueillons de futurs étudiants pour leur présenter les services de la bibliothèque. Aussi, parmi les nouveaux arrivants en L1, une grande partie connaît déjà la bibliothèque et a même bénéficié d'une visite. Comme nous avons senti une désaffection des primoarrivants sur la thématique " la BU je connais déjà », nous avons modifié l'organisation de ces visites afin de les adapter aux besoins recensés ou supposés ${ }^{5}$ en rendant notamment les étudiants plus actifs ${ }^{6}$.

Évaluer, surtout dans le domaine de la formation, consiste à être à l'écoute du public pour évoluer et s'adapter.

\section{LA DEUXIÈME ÉTAPE : L'ÉVALUATION QUALITATIVE}

L'évaluation qualitative passe en premier lieu par l'évaluation de la satisfaction. Nombreuses sont les bibliothèques qui utilisent un questionnaire en ligne ${ }^{7}$. C'est une étape nécessaire pour prendre en compte le ressenti des utilisateurs. Encore faut-il que les questionnaires soient adaptés et ne

4. Exemples : « Travailler à plusieurs ou à distance : les outils de travail collaboratif de Google »; "Soigner sa e-réputation »; « Trouver des articles d'actualité »; « Utiliser Orbis pour trouver un stage »; " Organiser une veille sur un sujet avec Scoop it », etc.

5. Il n'y a pas d'évaluation qualitative de ces visites, ces remarques se fondent sur l'observation des étudiants en séance, et sur la discussion avec les enseignants et avec eux.

6. 1795 étudiants accueillis en visite en 2011-2012 et 1062 en 2014-2015 mais le nombre d'étudiants formés est passé sur la même période de 1260 à 1954.

7. La BU d'Angers : < http://bu.univ-angers.fr/questionnaire-de-satisfaction > ; les BU de Lorraine : $<$ http://enquete.univ-lorraine.fr/directions/index.php?sid=63228\&lang=fr; les BU de Nantes : http://sphinx.univ-nantes.fr/bu/BU-EvalFormations16/BU-EvalFormations16.que?\&query=input\&st yle=3\&format=html\&lang=FR\&linktype=url\&linkurl=save.htm\&model=modele.htm\&utf8=1 > . 
mettent pas les formateurs dans une posture de défiance car la crainte subsiste que ce soit le formateur qui soit jugé. L'évaluation peut porter aussi bien sur le fond que sur la forme : on interroge le public sur sa satisfaction par rapport aux moyens, au moment, mais aussi aux supports et à l'animation de la formation.

Un autre moyen d'interroger le public sur sa satisfaction est d'utiliser l'enquête LibQual + qui mesure les écarts entre les attentes et la perception du service. Les questions principales sont standardisées et il n’y en a pas sur la formation des usagers. En 2010, lorsque la BU du Havre a lancé sa première enquête, la formation des usagers était l'un des enjeux (milieu du contrat 2008-2011), nous avons donc ajouté dans les questions optionnelles permises par cette enquête ces deux questions : " La bibliothèque me forme à la recherche, à l'évaluation et l'utilisation de l'information » et « les bibliothécaires m'expliquent comment utiliser efficacement les ressources en ligne (bases de données, revues, livres électroniques) ». L'analyse des résultats pour ces deux questions a permis de mettre en lumière l'insatisfaction du public de certaines UFR. Lors d'une intervention en Conseil des études et de la vie universitaire (CEVU) ${ }^{8}$ nous avons présenté ces résultats en les mettant en rapport avec le fait qu'aucune formation à la maîtrise de l'information n'était incluse dans les cursus des étudiants en Droit, Économie-gestion et Administration économique et sociale (AES), contrairement aux autres cursus. Cela a permis un dialogue avec la direction de l'UFR et les enseignants, pour aboutir à l'insertion de six heures de TD (dans le cadre global de la préparation au C2I) ${ }^{9}$ sur les ressources électroniques juridiques en L2 de Droit. En 2014, la deuxième enquête LibQual $+{ }^{10}$ a permis de mesurer pour ce public une très légère augmentation du niveau de satisfaction.

8. Ce conseil de l'université statue sur toutes les questions relatives à la formation, notamment l'élaboration des maquettes des diplômes.

9. Le domaine D4 du C2I porte sur « Organiser la recherche d'informations à l'ère du numérique », autour de l'acquisition de quatre compétences : recherche de l'information avec une démarche adaptée, évaluer les résultats d'une recherche, référencer une ressource numérique en ligne, organiser une veille informationnelle.

10. En 2014, une seule question optionnelle a été proposée sur la formation : " Les bibliothécaires apportent une aide à la recherche d'information, qui répond à un besoin immédiat et enrichit les compétences de l'usager pour ses recherches ultérieures ». 


\section{LA TROISIÈME ÉTAPE : MESURER L'IMPACT DES FORMATIONS DOCUMENTAIRES SUR LA RÉUSSITE ÉTUDIANTE}

L'évaluation de l'impact des formations sur la réussite des étudiants reste néanmoins difficile à mesurer. Il faut combiner à la fois les mesures statistiques, les mesures de satisfaction, les données observées ${ }^{11}$, et mener si possible des études sur le long terme.

On pourra s'attacher tout d'abord à mesurer la progression des étudiants dans l'acquisition de compétences ciblées, de façon subjective, ou selon une notation précise.

Cette année, une enseignante en gestion m’a sollicitée pour une séance avec ses étudiants de Master $1^{12}$. Sur la thématique globale de la logistique de distribution, par groupe de trois, les étudiants devaient choisir un sujet précis (exemple : « logistique de distribution et la reverse logistique »), réunir des sources, rédiger une synthèse de la littérature " sérieuse » (académique et/ou professionnelle), élaborer des hypothèses et les confronter au terrain (un entretien, des statistiques, un exemple), présenter ce travail complet et ses conclusions au cours d'un oral, et rédiger une synthèse comportant un plan détaillé de l'analyse effectuée, une bibliographie, une liste des sources interrogées et les questionnaires utilisés lors de l'étude. La séance portait sur la recherche d'articles de revues électroniques académiques ou professionnelles pour les aider à formuler leur problématique. L'enseignante ayant assisté à la séance en bibliothèque a pu lors de son propre enseignement évaluer les sources citées à la lumière de ce qui avait été présenté lors de la séance.

Un autre exemple : à l'occasion de l'organisation de notre première murder party ${ }^{13}$, nous avions dès le départ à l'esprit la nécessité de l'évaluation (notamment en raison de son coût en temps et en personnel). Nous avions des objectifs pédagogiques identifiés et nous avions donc conçu le questionnaire d'évaluation afin de mesurer leur atteinte. La question principale

11. Voir le Livre blanc: Qu'est-ce qui fait la valeur des bibliothèques ?, Afnor/CN46-8, 2016. [En ligne] : < http://portailgroupe.afnor.fr/public_espacenormalisation/AFNORCN46-8/Livre\%20 Blanc\%20fev2016.pdf >.

12. Le recrutement de ce Master est à plus de $50 \%$ extérieur. Les étudiants peuvent donc n'avoir suivi aucune formation préalable à l'utilisation des ressources de la BU.

13. Une murder party est une animation consistant à résoudre une énigme policière sous forme de jeu de rôle. 
("Avez-vous acquis des compétences grâce à la Murder Party ? ») était suivie de la liste des compétences à acquérir/mobiliser que nous avions ciblées dans la recherche des indices ( faire une recherche dans le catalogue et localiser un ouvrage en rayon ", " utiliser Factiva », " consulter mon compte lecteur », etc.). Le taux important de réponses à l'enquête et surtout les retours positifs ${ }^{14}$ sur l'importance du lien entre l'aspect « ludique » et l'aspect " pédagogique » nous ont confortés dans cette voie. L'évaluation est ici pour nous un levier de changement et d'innovation.

\section{Autoévaluation}

Une possibilité pour mesurer cet impact est de demander au public lui-même d'autoévaluer sa maîtrise des compétences dont l'acquisition constitue l'objectif pédagogique de la formation. Ainsi, la BU d'Angers préconise « la mise en place [...] en amont de la formation, de questionnaires (déclaratifs) et/ou de quiz pour connaître les acquis de [s] étudiants et adapter la formation en fonction. Les étudiants peuvent parfois avoir du mal à définir leurs besoins : dans ce cas, nous proposons des quiz avec des problèmes à résoudre, pour voir où ils en sont dans l'acquisition de leurs compétences informationnelles. $»^{15}$

$\mathrm{Au}$ Havre, en L1 dans le cours de méthodologie documentaire ${ }^{16}$, les étudiants sont invités en début d'année à remplir un bilan de leurs compétences en matière de "Stratégie de recherche documentaire ». Sur chaque item interrogé, l'étudiant indique son degré de performance. Cela permet à l'enseignante d'adapter son enseignement et surtout de mesurer les progrès réalisés dans l'année, une autre façon de mesurer l'impact de la formation.

14. Quelques commentaires : « Le côté ludique augmente l'investissement personnel pour l'apprentissage des divers outils », « On apprend en s'amusant », " C'est un moyen ludique de transmettre des informations », « Le fait que chaque indice soit lié à un aspect de la BU et qu'il soit expliqué était vraiment une bonne idée ! » et surtout un commentaire nous a particulièrement confortés sur l'utilité de ce genre d'action: " J'ai été surprise de devoir utiliser les services de la BU pour résoudre l'enquête et finalement très contente, cette soirée va vraiment m'aider dans mes recherches pour mon mémoire !».

15. Sur le blog professionnel BUApro, une série de billets sur « Méthodes Ux, active learning et formation des usagers » détaille un certain nombre d'actions mises en place sur l'évaluation des formations. [En ligne] : < http://bu.univ-angers.fr/node/1764 >.

16. Cet enseignement de 18 heures était assuré par une enseignante de documentation jusqu'en 2012. 
Ainsi, l'évaluation fonctionne à double sens. Il s'agit à la fois d'évaluer les étudiants (et de les noter), dans le cadre des enseignements qui font partie de leur cursus universitaire, et en même temps d'évaluer l'impact de la formation par ses résultats.

On peut espérer que les compétences informationnelles acquises vont être réinvesties, et par conséquent contribueront à démontrer l'impact des formations sur la réussite et l'insertion professionnelle des étudiants.

Plusieurs études récentes font ce lien ${ }^{17}$, en se basant sur l'analyse de cohortes complètes sur plusieurs années. Mais on peut sans doute commencer par des mesures plus simples.

\section{Évaluer un parcours documentaire}

Une première piste est l'introduction d'une question sur les formations documentaires, dans le questionnaire d'évaluation des enseignements. En effet, chaque année au Havre les étudiants doivent remplir un questionnaire pour évaluer d'une part leur formation, d'autre part chaque cours. De même, on pourra envisager d'introduire dans l'enquête d'insertion envoyée $a$ posteriori ${ }^{18}$ aux étudiants une interrogation sur le rôle éventuel de ces formations dans leur diplomation et leur insertion professionnelle ${ }^{19}$. Une enseignante de documentation intervenant dans un parcours documentation de Licence a souhaité elle-même engager cette démarche d'évaluation du parcours dans le cadre de la prochaine campagne d'accréditation. Une question s'intéresse notamment à l'évaluation de cet impact : "Quels apports et/ou compétences acquises vous aident, aujourd'hui, à la réussite de vos études ou de votre métier ? ». Les commentaires des étudiants soulignent essentiellement l'apport aux travaux ultérieurs de recherche et notamment de mémoire ${ }^{20}$, mais également de capacité de synthèse, d'analyse, de sélection et de présentation.

17. Voir l'étude de Dominique Papin qui fait la synthèse de la littérature sur L'impact de la bibliothèque sur la réussite des étudiants (décembre 2015).

18. Une enquête est envoyée après 24 mois.

19. Le lien entre maîtrise des bases de données juridiques et l'obtention d'un stage nous a très souvent été rapporté par des étudiants en Droit.

20. Par exemple : « Ce parcours m'aide beaucoup pour rédiger la bibliographie [et] me sert également pour savoir où me diriger lors de mes recherches car je connais des sites " fiables". " ou "Les méthodes de recherche sur les catalogues de bibliothèque et la méthode de bibliographie m'aident dans la confection de mon mémoire. » 
Le témoignage le plus complet met en valeur les apports généraux de cet enseignement :

" En définitive, il me semble que le parcours documentation a été un apport essentiel dans le cadre de la formation que j'ai suivie à l'université du Havre, car elle m'a appris à mieux penser, problématiser, classer, chercher, ce que n'offre pas un cursus général qui est trop spécialisé et qui oublie d'apprendre, peut-être, une méthode non spécialisée mais pourtant essentielle du point de vue du travail intellectuel. »

En conclusion, les formations à la maîtrise des compétences informationnelles faisant aujourd'hui partie intégrante des missions des bibliothèques, il convient de les évaluer afin de montrer en quoi elles peuvent être un maillon de la mission de l'université dans la réussite des étudiants et leur insertion professionnelle. Si toutes les actions ont leur importance, l'un des leviers est l'insertion de ces enseignements dans les diplômes afin qu'ils soient considérés comme faisant pleinement partie du cursus nécessaire à la réussite de l'étudiant. Mais dans tous les cas, des simples mesures de comptabilisation à l'étude de cohortes sur plusieurs années en passant par les enquêtes de satisfaction, les moyens sont nombreux pour évaluer les formations à la maîtrise de l'information dans les universités et les bibliothèques ont tout intérêt à s'en saisir pour alimenter le débat sur l'impact des bibliothèques, clé de leur existence en tant qu'acteur de la pédagogie à l'université. 


\section{2}

LE SERVICE PUBLIC POSTÉ EN BIBLIOTHĖQUE : COMMENT MESURER L'EFFICACITÉ D'UN SERVICE ORDINAIRE Muriel Amar et Guilaine Thuillart ET DISCRET ? L'EXPÉRIMENTATION DE LA MÉTHODE AMÉRICAINE READ À LA BPI

À l'origine de l'expérimentation à laquelle est consacrée cette contribution, se trouve une demande de la direction de la Bpi adressée aux deux services Études et recherche et Accueil des publics : conduire une semaine-test de l'accueil de façon à disposer d'indicateurs quantitatifs actualisés.

Cette semaine-test devait se consacrer à l'analyse des interactions menées en service public : le service public est posté à la Bpi et peut se définir par une unité de temps, entre 3 et 6 heures de bibliothécaire employées à répondre aux sollicitations des publics, et par une unité de lieu, matérialisée à la Bpi par des bureaux d'information.

\section{PAROLES DES USAGERS, PAROLES DES BIBLIOTHÉCAIRES : QUE CHOISIR ?}

En matière d'analyse des interactions, on peut distinguer deux grands types de méthode en fonction du point d'observation que l'on se donne : soit on se place du point de vue des usagers et on recueille leurs questions, soit on se place du point de vue des bibliothécaires et on recueille leurs réponses. Les questions posées peuvent être retranscrites de façon plus ou moins littérale ou peuvent être d'emblée, au moment du relevé, catégorisées dans une typologie prédéfinie, pratique la plus développée en bibliothèque. Plusieurs types de catégorisation peuvent être envisagés : thématique par sujet documentaire ou encore descriptive par type de question (question simple, question complexe, etc.) ; les typologies peuvent 
être plus analytiques, d'orientation cognitive ${ }^{1}$ ou sociologique ${ }^{2}$. L'avantage de ce type de catégorisation, c'est qu'elles permettent de produire facilement des données quantitatives. Cette approche repose cependant sur deux hypothèses fortes : on peut isoler la question d'un usager de son contexte de communication et l'usager est à même de formuler son besoin d'information de façon claire et définitive ; cette approche ne peut rendre compte de ce que produit la situation de dialogue usagers-bibliothécaires notamment par l'explicitation, la reformulation, etc. L'autre type de méthode envisageable consiste à placer le poste d'observation du point de vue des réponses apportées par les bibliothécaires : cette approche cherche à se situer au plus près de la situation de communication pour mesurer le travail d'adaptation à l'usager, le travail de reformulation réciproque, l'effort conjoint des acteurs. C'est une méthode qui prend pour hypothèse qu'une même question peut faire l'objet de réponses différentes, en fonction des interlocuteurs et du tour que prend l'interaction elle-même. Pour capter ce type de déplacement, ce sont des méthodes qualitatives qui sont privilégiées, séances d'analyse de la pratique ou encore entretiens longs, semi-directifs à l'exemple de l'étude qu'avait pilotée Anne-Marie Bertrand dans son ouvrage Bibliothécaires face au public ${ }^{3}$.

\section{MÉTHODE READ : LA NOTION DE « STATISTIQUES QUALITATIVES » OU LE MEILLEUR DES DEUX MONDES ?}

Initiée en 2002, la méthode d'analyse des interactions READ (pour Reference Effort Assessment Data) ${ }^{4}$ est le fruit d'un constat paradoxal établi par les bibliothèques d'étude américaines : si les services de référence, qui ont subi de plein fouet la concurrence d'Internet, enregistrent une baisse significative du volume de sollicitations, ils enregistrent, concomitamment, des demandes à traiter de plus en plus difficiles ou complexes, liées au sujet

1. Voir, sur ce point Bertrand Calenge, Les bibliothèques et la médiation des connaissances, Paris, Éditions du Cercle de la Librairie, 2015 (coll. Bibliothèques).

2. Par exemple, Jean-François Barbier-Bouvet, « La médiation facultative », in Jean-François Barbier-Bouvet, Martine Poulain, Publics à l'œuvre : pratiques culturelles à la Bibliothèque publique d'information du Centre Georges Pompidou, Paris, Éditions de la Bibliothèque publique d'information ; La Documentation française, 1986 (coll. Études et recherche), pp. 117-123.

3. Aux Éditions de la Bibliothèque publique d’information, 1995 (coll. Études et recherche).

4. Échelle d'évaluation des services de référence. 
de recherche comme au comportement des usagers, invités par les platesformes en ligne à développer une démarche de plus en plus autonome ${ }^{5}$. Pour les bibliothécaires regroupés au sein de l'Association américaine des bibliothèques d'étude, il ne fait aucun doute que si tout change, le contexte numérique comme le comportement des usagers, il faut aussi que les méthodes d'évaluation changent : on ne peut plus se contenter de relevés quantitatifs, il faut disposer d'une méthode plus qualitative, de type holistique qui capte " toutes les occurrences de l'activité de référence susceptibles de rendre compte des compétences, connaissances, techniques et outils nécessaires à mobiliser lors d'une interaction avec le public $»^{6}$. Bella Karr Gerlich propose alors une échelle de six niveaux, permettant au bibliothécaire de coder l'effort et l'expertise mobilisés, de les situer sur une échelle, évitant de comptabiliser de la même manière des interventions professionnelles de nature différente ${ }^{7}$.

En 2014, la bibliothèque de l'Institut d'études politiques de Paris est la première bibliothèque française à recourir à la méthode READ pour mener une analyse des interactions en service public. Après avoir proposé une traduction en langue française, la bibliothèque de Sciences Po Paris adapte l'échelle READ à ses besoins spécifiques, notamment en matière d'évaluation des formations nécessaires en service public posté.

La Bpi, de son côté, avait eu la chance de pouvoir expérimenter plusieurs types de méthode d'analyse des interactions en service public, à la fois quantitatives (notamment en 2003 et 2010) et qualitatives (voir supra et note 3). Nous avons souhaité, pour la campagne d'analyse 2015, tester une approche mixte. De ce point de vue, la méthode READ avec la notion qu'elle porte de " statistique qualitative " nous paraissait constituer un compromis intéressant permettant de disposer à la fois :

5. Bella Karr Gerlich, Lynn G. Berard, Jean McLaughlin, Gretchen Revie and Sue Liebold, "Using the READ Scale (Reference Effort Assessment Data): Qualitative Statistics for Meaningful Reference Assessment" (2008; Seattle: Washington) Proceedings of the 2008 Library Assessment Conference, Association of Research Libraries, Washington, 2008, pp. 361-389. [En ligne] : < http://docplayer. net/18078753-Proceedings-of-the-2008-library-assessment-conference.html >.

6. Ibid, p. 361.

7. Un site dédié, READScale $\odot$, met à disposition la méthode, des comptes rendus d'expérience et des publications. [En ligne] : $<$ http://readscale.org/ $>$. 
- de données quantitatives qui fournissent de précieux cadrages : nombre de réponses données par niveau d'expertise, bureau d'information, tranche horaire, etc. ;

- de données qualitatives permettant de rendre compte de l'interaction en tant que telle, en identifiant finement le rôle de la reformulation, de la connaissance de l'offre, de l'accompagnement de l'usager ainsi que les différents registres de réponse que peut donner le bibliothécaire.

\section{ADAPTATION DE LA MÉTHODE READ AU CONTEXTE BPI : UN TRAVAIL COLLABORATIF}

Ce qui a rendu envisageable l'utilisation de l'échelle READ à la Bpi, c'est bien sûr la composition du public de la bibliothèque qui compte, selon les données récentes de l'enquête générale de fréquentation, un peu plus de $60 \%$ d'étudiants ${ }^{8}$. Nous savions que la méthode READ élaborée dans le cadre des bibliothèques d'étude pouvait bien convenir à cette part des interactions relevées aux bureaux d'information ; mais nous devions également adapter la grille américaine pour rendre compte des échanges que nous développions avec nos autres publics : actifs, retraités, ou encore demandeurs d'emploi ${ }^{9}$.

L'adaptation a porté principalement sur deux aspects.

\section{choix des niveaux de l'échelle américaine READ originale}

Le niveau 1 de l'échelle américaine READ se définit par la négative : c'est un niveau de réponse qui ne fait appel à aucune ressource ou compétence documentaire. S'il permet de comptabiliser le nombre de réponses non documentaires, il ne permet pas de les spécifier ; or, il nous semblait important de connaître la nature des réponses concernant les services pratiques apportés aux usagers. Des volumes importants de sollicitations portant sur la localisation des espaces (cafétéria, toilettes, etc.), le

8. Enquête générale de fréquentation 2015 à la Bpi, réalisée par la société TEST. [En ligne] : < http:// www.bpi.fr/etudes/observation-des-publics-de-la-bpi/barometre >.

9. Ces trois catégories représentent $32 \%$ du public en 2015. 
fonctionnement de la bibliothèque (horaires, modalités de prêt), ou encore les services d'impression, de reproduction ou d'usages des postes informatiques, peuvent signaler une communication confuse de l'institution ou encore un manque de visibilité de l'offre : il sera d'autant plus facile de remédier à ces dysfonctionnements qu'ils seront précisément circonscrits ${ }^{10}$. Nous avons donc transformé le niveau 1 de l'échelle READ en une rubrique de notre grille d'analyse des interactions constituée d'items prédéfinis à coder.

FORMULAIRE DE CODAGE

\section{GRILLE D'ANALYSE} DES INTERACTIONS

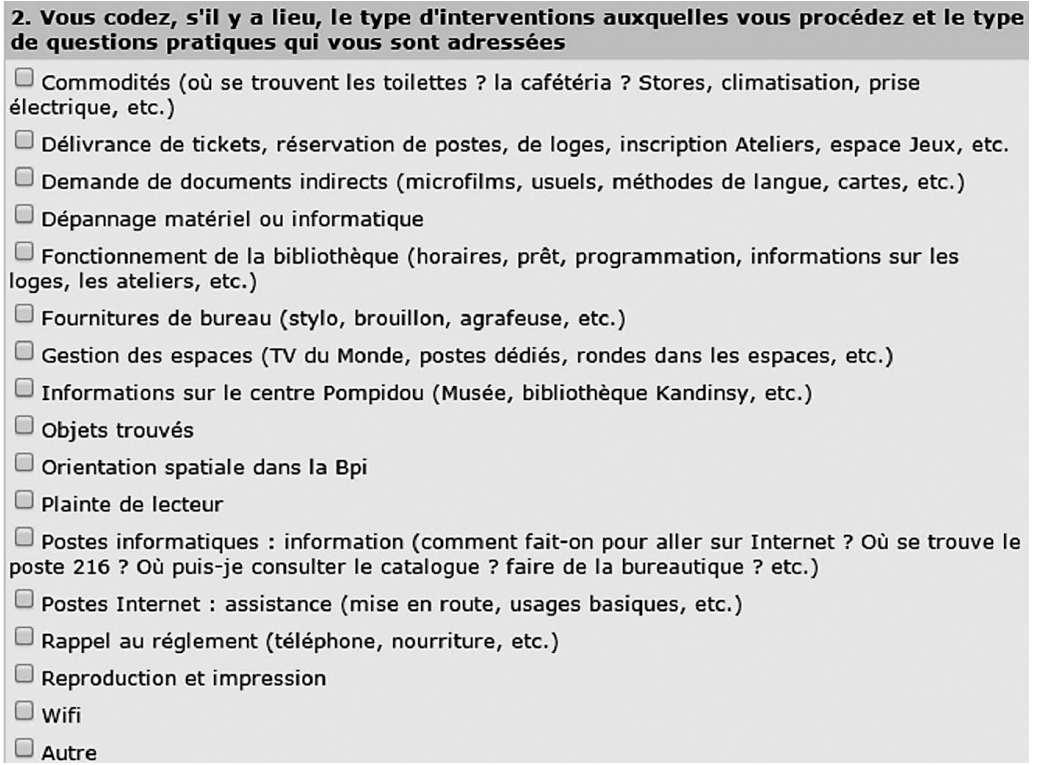

Légende : rubrique 2 du formulaire de codage des interactions en service public à la Bpi. Source : Bibliothèque publique d'information.

10. Voir, sur ce point, Jean-Philippe Lamy, "Évaluer un service de référence », Bulletin des bibliothèques de France, 2001, n 4, pp. 82-88. [En ligne] : < http://bbf.enssib.fr/consulter/bbf-200104-0082-001 >. 
Nous avons uniquement retenu les niveaux 2 à 5 de l'échelle READ pour qualifier l'expertise et l'effort mis en œuvre par les bibliothécaires, en excluant le niveau 6 qui correspond dans la grille américaine à un travail d'accompagnement documentaire personnalisé auprès de chercheurs, hors du ressort de la Bpi.

Nous avons spécifié les critères de passage d'un niveau à l'autre en privilégiant moins le recours aux ressources documentaires (type bases de données), déterminant dans l'échelle READ originale, que l'engagement du lecteur dans l'entretien documentaire. En effet, le recours aux ressources documentaires est désormais incontournable et ne paraissait pas constituer, pour la Bpi, un critère discriminant. En revanche, ce qui constitue à nos yeux un paramètre important de la médiation en bureau d'information est la qualité de l'échange que nous parvenons à créer avec les usagers, qui peuvent se sentir peu légitimes à solliciter un accompagnement documentaire ou pour qui, au contraire, le temps de la médiation peut être perçu comme un frein, une sorte de "temps perdu », voire un obstacle à l'obtention des informations dont ils ont besoin. De ce point de vue, nous avons distingué les réponses documentaires apportées sans besoin de reformulation (niveau 2 correspondant par exemple à la recherche d'une référence déjà fournie par l'usager) des réponses de niveaux 3 à 5 nécessitant ce temps de reformulation et d'échange. Si l'usager souhaite simplement déléguer au bibliothécaire la recherche dans les collections ou les ressources documentaires, nous considérons cette réponse de niveau 3 (avec reformulation, sur un mode d'assistance) ; s'il souhaite mener sa recherche seul, nous l'initions aux outils adaptés à sa demande en visant son autonomie (niveau 4 avec reformulation, en mode formation) ; enfin, si l'usager considère le bibliothécaire comme un partenaire pour mener sa recherche, lui fournissant les pistes déjà explorées et le maximum de précisions concernant sa demande, alors nous situons cette interaction au niveau 5 (avec reformulation sur un mode de coopération). 


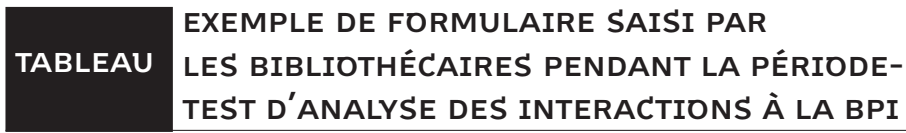

\begin{tabular}{|c|c|}
\hline Variable & Données \\
\hline Services documentaires rendus & $\begin{array}{l}\text { Niveau } 5: \text { réponse avec reformulation } \\
\text { en mode coopération }\end{array}$ \\
\hline Services pratiques & $\begin{array}{l}\text { Fonctionnement de la bibliothèque } \\
\text { (horaires, prêt, programmation, } \\
\text { informations sur les loges, les ateliers, } \\
\text { etc.) /Orientation spatiale dans la Bpi }\end{array}$ \\
\hline Accompagnement & $\begin{array}{l}\text { Accompagnement social (dialogue de } \\
\text { convivialité, empathie, etc.) }\end{array}$ \\
\hline Localisation & Musique \\
\hline Horaire & $16 \mathrm{~h}-18 \mathrm{~h}$ \\
\hline Commentaire libre & $\begin{array}{l}\text { Un usager qui avait des demandes } \\
\text { précises sur le design graphique et } \\
\text { ensuite la typographie dans les données } \\
\text { visuelles (écrans). Demandes très pré- } \\
\text { cises avec une volonté de s'informer au } \\
\text { maximum de toutes les possibilités et } \\
\text { offres de la bibliothèque. }\end{array}$ \\
\hline Date de saisie & 06 novembre 2015 \\
\hline
\end{tabular}

Source: Bibliothèque publique d'information.

Ces adaptations apportées à l'échelle originale READ permettent de préciser le niveau de réponse documentaire apportée à l'usager tout en signalant les renseignements pratiques fournis au sein de la même interaction, grâce à la transformation réalisée sur le niveau 1 de l'échelle READ déplié sous la forme d'une liste d’items pratiques prédéfinis. 


\section{Décrire l'accompagnement des usagers}

La seconde adaptation de l'échelle READ opérée à la Bpi concerne l'ajout d'une rubrique qualifiant la nature de l'accompagnement réalisée auprès des usagers. En effet, la diversité des publics accueillis, des plus scolaires aux plus précaires, nous a incités à rendre compte de la prise en charge de ces singularités par les personnels postés. De ce point de vue, nous avons prévu une rubrique permettant de signaler des interactions, documentaires ou non, qui ont donné lieu par exemple à un dialogue de convivialité, à dimension sociale plus ou moins importante (un usager expose longuement les problèmes rencontrés avec son propriétaire et souhaite identifier des informations juridiques pour faire entendre sa position) ou encore à un dialogue plus pédagogique par lequel le bibliothécaire peut apporter une aide méthodologique pour un travail scolaire ou ses compétences d'écriture pour un courrier officiel. Cette écoute bienveillante ou cette aide méthodologique est prise en charge par le bibliothécaire en tant que représentant d'une institution culturelle, qui symbolise parfois, aux yeux des usagers, une instance légitime de seconde chance leur permettant d'accéder à un service public qui peut avoir fait défaut dans d'autres cadres (le lycée pour les lycéens, les services sociaux pour les plus démunis par exemple).

Là encore, l'existence de cette rubrique, facultative comme celles précédemment exposées, permet de décrire, sous plusieurs aspects et avec un seul formulaire de codage, la multiplicité des savoir-faire mobilisés par les bibliothécaires en contact avec les publics.

Ces deux types d'adaptation, qui captent finement les spécificités de l'accueil aux bureaux d'information à la Bpi, n'ont pu se faire qu'avec la contribution, active et engagée, des bibliothécaires. D'emblée appréhendé par les deux pilotes du projet comme une co-construction, ce travail d'adaptation a engagé tous les collègues coordonnateurs des bureaux d'information (dix professionnels) et, au-delà, tous les collègues intéressés à travers des séances de présentation-discussion et une période de formation-test proposée à tous les personnels postés pendant quinze jours.

Cette gestion de projet, sur un mode collaboratif, a permis de faire d'un outil d'auto-évaluation de l'activité des bibliothécaires en service public, délicat à proposer dans un contexte de refonte des services au public à la 
Bpi, un instrument d'analyse de la pratique, riche de découvertes et très valorisant ${ }^{11}$.

\section{MISE EN CEUVRE ET DISCUSSION}

Ce mode de gestion de projet, collaboratif, a sans doute contribué à l'engagement important et enthousiaste des collègues pendant toute la durée du recueil, qui s'est déroulé entre le 4 et le 13 novembre $2015^{12}$. Les deux copilotes, épaulées par un vacataire, étaient présentes presque en continu pendant ces 93 heures d'accueil du public (41 061 entrées comptabilisées sur la période) pour répondre aux questions des collègues, rappeler les consignes de saisie, faire face aux impondérables (panne informatique par exemple), etc.

Pendant la période-test, ce sont 145 personnels postés - 117 titulaires et 28 vacataires - qui ont saisi 6739 formulaires décrivant autant de situations d'interaction. Ces situations d'interaction retracent 19385 interventions professionnelles différentes ${ }^{13}$ : ce qui signifie qu'une interaction fait l'objet en moyenne de trois modalités de réponse différentes de la part des bibliothécaires - réponse documentaire, renseignements pratiques, accompagnement des publics, distribution de tickets d'accès à Internet, etc. Le volume des données recueillies autorise à tester leur robustesse statistique : y a-t-il eu des erreurs de codage ? Des surcodages ? Des incompréhensions de la grille d'analyse proposée ? La base de données constituée a été nettoyée de toutes les scories détectables (incohérence de saisie sur des horaires ou des niveaux de codage par exemple), qui ont touché un peu moins de 200 questionnaires, soit $3 \%$ des données recueillies. Des tests ont été réalisés qui ont montré une stabilité des réponses d'un jour à

11. Deux séances de débriefing ont été organisées après l'enquête de façon à recueillir l'expérience des bibliothécaires : elle a été, pour ceux qui se sont exprimés, jugée " gratifiante », « ludique », permettant « de prendre conscience de tout ce que l'on fait car spontanément : on ne s'en rend pas compte », constituant une "prise de distance intéressante par rapport à sa pratique », comblant un manque : «On n'a jamais autant parlé du SP ».

12. Initialement prévu pour durer quinze jours, le test a été interrompu suite aux attentats du 13 novembre 2015 à Paris.

13. Ces données chiffrées, qui peuvent paraître importantes, constituent pourtant une estimation basse, certaines interactions n'ont pu être codées car oubliées, surtout dans les cas où le bibliothécaire enchaîne les réponses à donner. 
l'autre, d'un bureau à l'autre, d'une rubrique à l'autre et la première surprise a été de relever une véritable consistance des données, révélant une constance du service public à la Bpi.

La méthode READ invitant le bibliothécaire à situer sa propre réponse sur une échelle de difficulté repose à la fois sur une analyse subjective de son activité et sur un outil objectif, car contraint, de modélisation. L'écueil, bien connu en documentation, de la variabilité des pratiques (comme de nombreuses études sur l'indexation ont pu le montrer) peut difficilement trouver sa place dans ce type d'analyse de la pratique où, finalement, les paramètres de variation peuvent être contrôlés ${ }^{14}$.

Paradoxalement, avec plus de 2000 commentaires libres accompagnant les formulaires codés pour retracer les questions posées et leurs évolutions, nous avons le sentiment que l'intérêt porté aux réponses des bibliothécaires constitue finalement une méthode bien plus fine pour appréhender les besoins des usagers que celle consistant précisément à leur donner directement la parole ${ }^{15}$.

Même si la méthode READ appliquée à la Bpi ne rend pas compte de tout ce qui se passe à la bibliothèque entre professionnels et usagers, elle rend visible l'épaisseur de ces échanges simples et ordinaires où un professionnel cherche à rendre facile et naturelle la rencontre avec des univers documentaires et institutionnels souvent complexes, contraignants et parfois peu engageants : ce sont ces « vertus stratégiques de la discrétion des services documentaires $»^{16}$ auxquelles notre enquête s'est finalement intéressée.

14. Les collègues américains ont particulièrement étudié, avec le concours de 14 services de référence en BU, les effets que pouvait produire la subjectivité de l'analyste avant de confirmer la viabilité de la méthode : Bella Karr Gerlich, Lynn G. Berard, “Testing the Viability of the READ Scale(C) (Reference Effort Assessment Data): Qualitative Statistics for Academic Reference Services”, College and Research Libraries, March 2010, 71 (2), pp. 116-137.

15. C'est d'ailleurs le titre de la communication adoptée par Catherine Fortier et Lyne Welsh " Traduire l'effort et l'expertise du personnel pour mieux évaluer les besoins des usagers », Congrès des milieux documentaires du Québec, 18-20 novembre 2015. [En ligne] : < https://milieuxdoc. ca/actes-des-conferences-fr1199.html >.

16. Titre de l'article d'Isabelle Le Bis et Béatrice Vacher paru dans Documentaliste-Sciences de l'information, 2006, 43 (3), pp. 200-208. 


\section{3}

\section{ÉVALUER LES ATTENTES ET PERCEPTIONS : L'OUTIL LIBQUAL + ${ }^{\circledR}$, SES AVANTAGES ET SES LIMITES}

par

Frédéric

Brodkom

\section{L'ENQUÊTE LIBQUAL}

Développée aux États-Unis en 2000 par l'Association of Research Libraries $(A R L)^{1}$ et la Texas A \& M. University, LibQUAL $+{ }^{\circledR}{ }^{2}$ est une enquête en ligne centrée sur 22 questions qui mesurent les attentes, souhaits et perceptions des utilisateurs de bibliothèques par rapport à l'accueil, aux espaces et aux ressources documentaires. Chaque question est une affirmation - « le personnel m'inspire confiance ", " les locaux sont accueillants et confortables ", etc. - et le répondant est invité à attribuer trois valeurs, chacune sur une échelle de 1 à 9 : la valeur minimum de qualité qu'il espère du service énoncé - l'attente minimale -, la valeur maximum de qualité qu'il attend pour ce service - son souhait -, et la valeur qu'il juge représenter sa perception, son vécu ressenti par rapport à l'affirmation. Le répondant calibre donc sa perception de la qualité en regard de sa zone de confort entre attente minimale et souhait le plus élevé. L'enquête mesure aussi divers points de satisfaction (ou non) et les fréquences d'usages (de la bibliothèque, des services en ligne). Il y a enfin des questions démographiques : niveau d'étude ou catégorie de personnel, discipline, bibliothèque de référence, tranche d'âge, sexe. Elles permettent de réaliser des analyses plus fines par catégorie, discipline, etc. Pour terminer, l'occasion est donnée d'émettre un commentaire libre. Deux sessions d'enquête sont ouvertes chaque année, 125 institutions dans 14 pays y ont participé ${ }^{3}$ en 2015. En France, la première expérience a été celle de HEC qui la renouvellera en 2006, Lyon I suivra en 20074. En

1. Association of Research Libraries : < http://www.arl.org/ >.

2. Pour la suite de ce texte la version simplifiée du terme " LibQUAL » sera utilisée en lieu et place de « LibQUAL+® » : < http://www.libqual.org >.

3. < https://www.libqual.org/documents/LibQual/publications/2015_LibQUAL_Highlights.pdf >.

4. LibQUAL-fr : < http://libqual-fr.pbworks.com >. 
Suisse romande ${ }^{5}$, l'École polytechnique fédérale de Lausanne (EPFL) a été la première à lancer l'enquête en 2011 et en Belgique francophone, l'université catholique de Louvain (UCL), l'université libre de Bruxelles (ULB), l'université de Liège (ULg) et après l'université de Namur (UNamur) ont participé ensemble aux enquêtes de 2009 et 2015.

\section{MESURER LES EFFETS DE SON ACTION}

Les données analytiques fournies par les $43^{6}$ questions de l'enquête LibQUAL sont désormais entrées dans les tableaux de bord de gestion de bon nombre de bibliothèques. Au-delà des données bibliométriques classiques (surfaces, nombre d'ouvrages, etc.), décrivant donc l'input, et de l'analyse des processus qui nous fournissent des éléments sur les activités des bibliothécaires, il nous faut désormais aussi suivre, dans nos tableaux de bord, les effets de notre action, l'output. Les questions de LibQUAL permettent de percevoir le ressenti de nos usagers dans ces trois composantes de l'audit qualité : l'offre - la bibliothèque possède les ressources électroniques dont j'ai besoin... -, les actions - les usagers bénéficient d'un service personnalisé... -, et les impacts - la bibliothèque me rend plus efficace dans mes activités universitaires...

Le but ici ne sera pas de réécrire ce qui l'a déjà été sur LibQUAL mais de prendre du recul, après dix années d'utilisation en France, pour évaluer l'intérêt de cet outil mais aussi en identifier les limites, à savoir ce qu'il n'apporte pas ou mal dans ses promesses de compréhension des publics.

\section{L'INTÉRÊT D'UTILISER LIBQUAL}

L'objectif ici n'est pas de faire l'apologie de LibQUAL, mais de montrer la puissance des services apportés par LibQUAL, l'intérêt de participer à

5. Enquête de satisfaction de la Bibliothèque de l'EPFL : < http://libqual-fr.pbworks.com/f/ Page\%20WEB_definitive.pdf $>$.

6. Le questionnaire de base est constitué de 22 questions, auxquelles 5 questions locales sont ajoutées pour les consortia. Il y a en plus 8 questions de satisfaction et 3 questions sur les fréquences d'usage. Enfin, le questionnaire se termine par les 4 questions démographiques et 1 possibilité de commentaire libre. 
un projet collectif et la qualité des outils d'interprétation. Les candidats à l'aventure garderont à l'esprit que la participation à l'enquête doit être un projet d'équipe et qu'elle a un coût : inscription à 3200 dollars américains la session, coût de la promotion, heures de travail d'analyse des résultats, etc.

\section{LE BENCHMARKING}

Chacune des deux sessions annuelles de l'enquête est menée conjointement avec un même questionnaire dans plus d'une centaine de bibliothèques dans le monde. Dans leur manuel de procédures 20157 David Green et Martha Kyrillidou précisent que LibQUAL c'est plus de 1200 bibliothèques et plus de 1300000 répondants depuis 2000. Dans chaque pays ou communauté linguistique, il est rare qu'une bibliothèque soit la seule ou la première à se lancer dans LibQUAL. Sachant aussi que les gestionnaires doivent fournir les chiffres de leur bibliothèque en termes de budget, cadre d'effectif, population desservie, etc. et que ces données, comme le rapport d'enquête standardisé fourni par l'ARL, sont librement accessibles aux bibliothèques participantes de l'année en cours, LibQUAL permet de se comparer à d'autres bibliothèques de même taille et budget. Même si l'outil permet avant tout d'analyser la qualité via le regard porté par ses propres publics, il est donc aussi un outil très puissant de benchmarking national et international. Les bibliothèques qui analyseront les résultats de consœurs équivalentes et partageront avec elles les stratégies de succès ou les problèmes ne pourront qu'en tirer des enseignements pour améliorer leurs services, espaces et accès aux ressources.

\section{UN TRAVAIL COLLABORATIF}

À l'échelle nationale, ou transnationale dès lors qu'une langue commune est partagée, l'expansion de LibQUAL a favorisé la naissance de communautés de pratique. Au début, l'ARL souhaitait réduire le nombre de traductions des questions et canevas démographiques en favorisant le regroupement d'institutions en consortia avec questionnaires uniques. En bonus donc, les

7. David Green and Martha Kyrillidou, Procedures Manual, LibQUAL+®, Washington DC, Association of Reserach Libraries, 2012. Le copyright est bien de 2012, mais c'est le manuel toujours en application pour la session 2015. 
consortia ont la possibilité de définir cinq questions supplémentaires permettant d'évaluer des services particuliers ou de questionner sur des thématiques non reprises dans le questionnaire (horaires, formations, etc.). Des groupes de travail se sont mis en place pour animer ces consortia (Libqual-fr en France, GT LibQUAL de la Bibliothèque interuniversitaire de la Communauté française de Belgique [BICfB] en Belgique) et ont accumulé de l'expérience. Toute nouvelle bibliothèque participante peut donc facilement se raccrocher à ce travail collaboratif et au paramétrage de son consortium national.

\section{UNE MESURE CONJOINTE DES ATTENTES ET DE LA PERCEPTION}

La méthodologie de LibQUAL s'inspire d'expériences antérieures développées dans le privé qui placent le consommateur au cœur du processus d'évaluation ${ }^{8}$. Le répondant est invité pour chaque question à donner sa perception en même temps qu'il nous informe sur ses attentes et souhaits : " les locaux de la bibliothèque incitent à l'étude. Sur une échelle de 1 à 9 ... quel est le minimum que j'attends des locaux pour m'inciter à étudier ? Quelle est la valeur qui reflète ce que je souhaite que ces locaux soient pour qu'ils m'incitent à bien y étudier ? Quelle est la valeur de mon vécu ou de mon observation de ce que ces locaux sont comme lieux qui m’incitent (ou pas) à y étudier ». Si je fixe mes attentes pour un hôtel entre 4 et 5 étoiles et qu'au terme de mon séjour j'estime qu'il en vaut 3, j'exprime un mécontentement. Si on me demande seulement combien d'étoiles je lui accorde, la seule réponse « 3 » n'exprime pas ma déception car je laisse à l'interrogateur la possibilité de croire que 3 est une bonne évaluation. Sachant que chaque individu a ses propres critères de qualité, lui permettre de fixer lui-même sa zone de confort avant d'exprimer sa perception est la méthode la plus efficace de mesure de la qualité.

\section{UNE RÉCOLTE ET MISE À DISPOSITION DES DONNÉES OPTIMISÉES}

L'enquête LibQUAL est une enquête en ligne et l'ARL offre un outil de récolte, de stockage, de traitement et de diffusion des données. Une fois l'enquête clôturée, souvent après 3-5 semaines, l'ARL fournit un rapport

8. Colleen C. Cook and Fred M. Heath, "Users's perceptions of library service quality: A 'LibQUAL+TM' qualitative study”, Library trends, 2001, 49, pp. 548-584. 
standard qui développe les analyses par groupes de répondants (bacheliers, masters et doctorats, enseignants et chercheurs). Parallèlement, les fichiers CSV (pour comma-separated values) avec l'ensemble des réponses permettent au gestionnaire de faire le tri et l'analyse des données dans tous les découpages possibles : " que pensent les étudiantes en Master 1 de psychologie de la qualité des locaux pour travaux de groupes dans leur bibliothèque de psychologie, quel est l'avis des chercheurs en agronomie sur l'accès à domicile aux ressources électroniques, etc. " L'ensemble du processus est optimisé pour faciliter le travail d'analyse et réduire l'investissement technique et humain des bibliothèques participantes.

\section{L'OUTIL DE COMMUNICATION}

La représentation classique des résultats est celle des diagrammes en forme de radar (voir le diagramme p. 124).

Les questions forment les 22 rayons d'un cercle, chacun gradué jusqu'à 9 , où les 3 valeurs sont marquées. En reliant les attentes minimales, on définit une aire blanche au cœur du radar tandis qu'avec la valeur maximale du souhait on délimite une aire gris clair au-dessus de la première. C'est l'espace de confort des attentes. Lorsqu'on relie les 22 points qui marquent la perception, on identifie des aires dans ou autour de la zone gris clair : blanc moucheté si la perception est inférieure à l'exigence minimale, gris moyen si la perception est dans la zone de confort et noir si la perception dépasse le souhait. Le visuel parle de lui-même : plus il y a de gris moyen ou noir, plus on est en adéquation avec son public, plus il y a de blanc moucheté plus des efforts sont à réaliser pour rencontrer ses exigences. Chaque sous-groupe peut ainsi être caractérisé par un radar dont la valeur pédagogique facilite la communication.

Les tableurs permettent bien d'autres représentations, à chaque analyste d'identifier ce qui est le plus parlant pour exprimer son propos. L'adequation gap, valeur qui sépare l'attente minimale de la perception peut être présenté sous forme de courbes pour les 22 questions tandis que les radars peuvent être remplacés par des diagrammes à barre et curseur, la barre allant du minimum au souhaité avec sur le même axe, un curseur qui représente la perception. 


\section{DIAGRAMME}

REPRÉSENTATION EN RADARS DES RÉSULTATS DE LIBQUAL

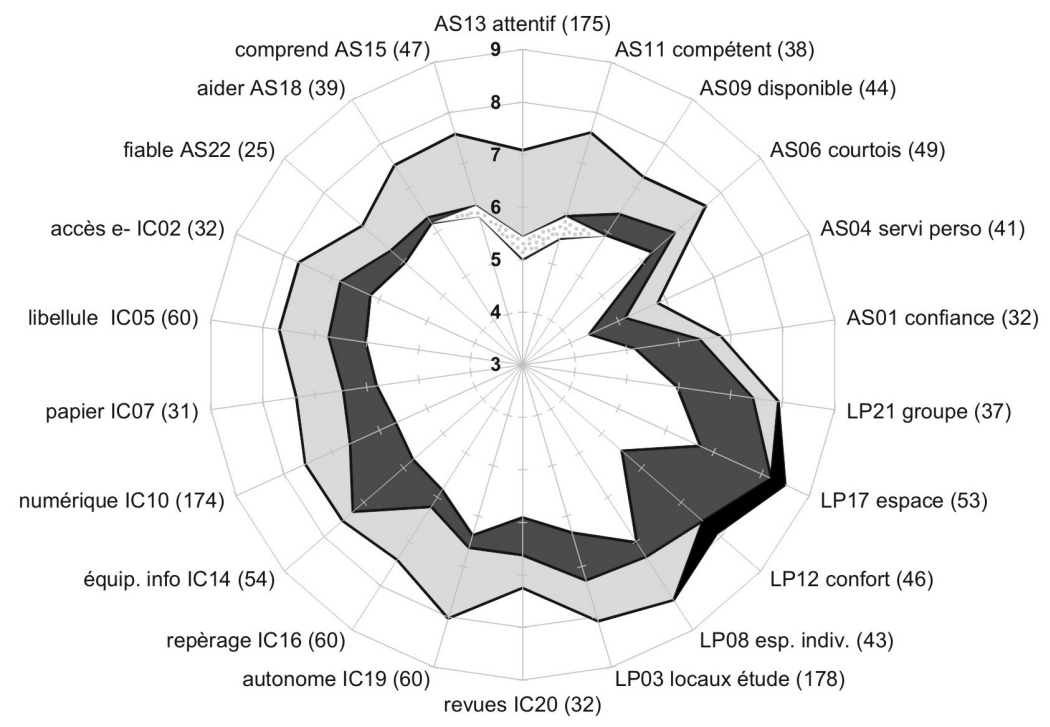

Source : Frédéric Brodkom, UCL/BST 2015.

Attente minimale :

à la limite supérieure de la zone blanche intérieure.

Souhait (désir) maximal :

à la limite supérieure de la zone en gris clair (la seconde couronne).

Niveau de perception :

> zone blanc moucheté : la perception est inférieure à l'attente minimale (dans le secteur Nord entre AS15 et AS11) ;

> zone gris moyen : la perception est supérieure à l'attente minimale (la première couronne) ;

> zone noire : la perception est supérieure au souhait/désir maximal (dans le secteur Sud-Est). 


\section{UNE ANALYSE DES CHANGEMENTS}

L'outil LibQUAL est enfin un outil qui s'inscrit dans la durée. Chaque année, la bibliothèque peut à nouveau mener l'enquête dont les questions sont inchangées. Elle peut donc suivre l'évolution dans le temps des attentes et perceptions de ses publics mais aussi, mesurer l'effet des mesures correctives réalisées. La stabilité dans le temps de LibQUAL est un atout majeur en termes d'analyse des impacts, voire en termes d'analyse prédictive des tendances en matière d'exigence des publics et de qualité.

\section{LES LIMITES À L'USAGE DE LIBQUAL}

L'ARL n'a jamais prétendu que LibQUAL répondait à tous les défis en termes d'évaluation et on pourrait établir une longue liste de ce que LibQUAL ne fait pas en termes de connaissance des publics, mais tel n'est pas notre but. Ci-après, nous poserons néanmoins quelques balises qui méritent une attention particulière lorsqu'on utilise ou interprète les résultats de LibQUAL.

\section{LES ÉNONCÉS DU QUESTIONNAIRE}

Le questionnaire standard de LibQUAL a été réalisé en 2000, il y a donc 16 ans et, à quelques détails près, il n'a pas changé depuis. Si nous pouvons y voir l'intérêt de l'évaluation du changement, il est évident que des sujets de l'actualité des bibliothèques ne sont pas évoqués : horaires, learning center, dépôts institutionnels, réseaux sociaux, prêt entre bibliothèques, médiation des contenus, etc. Ces sujets sont parfois abordés dans les 5 questions de consortia mais leur récurrence incite à penser qu'ARL pourrait les intégrer dans le corpus standard qui permet le benchmarking.

\section{LE MODE D'INTERROGATION QUI DISSUADE PLUS QU'IL N'ATTIRE}

L'expérience le démontre, les taux d'abandon en cours lors de la participation effective à l'enquête et de réponses non valides sont nombreux. À l'UCL en 2015, pour 5991 accès enregistrés au questionnaire, seules 2974 réponses sont complètes et valides ! La moitié des personnes qui ont accédé à l'enquête ont renoncé à y répondre, ont abandonné en cours ou 
ont mal répondu au point d'invalider leurs réponses. Le mode d'interrogation qui consiste à demander 3 valeurs pour chaque affirmation n'est pas très compréhensible et l'interface qui n'évolue guère vers plus de convivialité ne facilite pas cette compréhension nécessaire. Certains énoncés sont redondants, pour vérifier que le répondant prend la peine de les lire et répond correctement, et le questionnaire dans sa version complète est trop long. Conscient de cela, l'ARL propose depuis peu un questionnaire LITE qui réduit le nombre de questions pour chaque participant en en proposant une partie de manière aléatoire, avec une garantie que toutes les questions seront posées si le nombre de répondants est suffisant. Le questionnaire LibQUAL n'est donc pas simple, et nécessite de gros efforts pour en faire la promotion et l'expliquer clairement aux utilisateurs afin que ceux-ci puissent se l'approprier pleinement. Cette appropriation passe aussi par l'habitude, la répétition annuelle de l'enquête permet d'augmenter la participation. La généralisation de la version allégée LITE et un relooking de l'interface qui donnerait envie de participer sont deux autres pistes que l'ARL devrait d'urgence explorer.

\section{LA REPRÉSENTATIVITÉ DES SOUS-GROUPES}

LibQUAL se réalise soit par l'envoi du lien du questionnaire à une population échantillonnée, soit par la communication du lien à l'ensemble de la population ciblée, à savoir les publics qui fréquentent la bibliothèque. C'est souvent cette seconde méthode qui est utilisée. La question se pose alors de la représentativité des réponses par sous-groupes. L'exemple de l'UCL a montré en 2015 que pour le sous-groupe des masters et doctorants, en prenant les extrêmes, le taux de participation a été de $25,1 \%$ en Langue et lettres contre 2,6\% en Sciences médicales. On ouvre ainsi une brèche aux détracteurs qui évoquent la trop faible représentativité des réponses. La question de la représentativité (ou non) n'est pas propre à LibQUAL mais elle pose les limites de ce qui est permis de faire en matière de comparaison et d'interprétation. Ce sera aux analystes d'exposer les faits en toute transparence et de laisser les bibliothécaires tirer les conclusions qu'ils jugeront utiles et nécessaires au développement de la qualité.

En tenant compte des limites évoquées, l'outil LibQUAL est indéniablement le meilleur protocole d'enquête en ligne pour évaluer les exigences, 
attentes et perceptions de publics des BU en matière de qualité. C'est un outil clé en main, largement répandu et connu, qui apporte une contribution effective à la connaissance des publics et la gestion de la qualité. Mais, comme je l'ai présenté à Paris en $2015^{9}$, d'autres types d'enquête doivent être menés en parallèle et en continu : enquêtes d'observation (dite Sweeping) pour identifier les usages des différents lieux de la bibliothèque, entretiens personnalisés de publics sélectionnés sur des thématiques de fonds, questionnaires de positionnement de la bibliothèque et de ses services en regard d'autres alternatives, etc. Dans tous les cas, et à l'instar de ce qui se fait dans les entreprises, les bibliothèques qui n'ont pas encore développé les moyens humains et financiers dans le contrôle systématique de la qualité devront tôt ou tard s'y mettre avant de perdre le respect de leurs publics, car ce sont bien leurs avis, leurs besoins et leurs usages qui doivent guider nos politiques du moment et notre investissement pour demain. 


\section{4}

ÉVALUER LA CONSULTATION SUR PLACE ET L'USAGE DES ESPACES : Cécile Touitou LE CAS DE LA BIBLIOTHÈQUE DE SCIENCES PO PARIS

Pour décrire la bibliothèque de Sciences Po, on pourrait dire qu'en 2015 elle comptait 643000 livres, 5500 e-books, 15250 périodiques, 20400 périodiques en ligne, $23 \mathrm{~km}$ de rayonnages, 12768 lecteurs, 962155 visites et 167644 prêts.

Ces chiffres peuvent donner un premier aperçu de la bibliothèque. Mais, au-delà des données générées par le SIGB (les prêts) ou par les compteurs d'accès (le nombre de visites), la bibliothèque a été longtemps impuissante à connaître l'usage des collections offertes en salles de lecture (77 000 livres en accès direct). Seules $10 \%$ des collections imprimées sont directement accessibles en salles. La composition de ce fonds et l'usage qui en est fait par les lecteurs constituent donc incontestablement des enjeux forts qui déterminent en partie l'attractivité de l'établissement auprès de ses usagers.

Ces ouvrages, soigneusement sélectionnés par les responsables thématiques, ont l'ambition d'être au cœur des intérêts des lecteurs. La charte documentaire rédigée en 2015 par le département du Développement documentaire précise à leur sujet : "Sont mis en priorité en accès direct les documents prescrits par les enseignants, le plus souvent des livres. Ces documents sont prêtés pour une courte durée pour favoriser leur rotation. »

\section{PASSER DE LA MESURE DES COLLECTIONS AUX USAGES DES PUBLICS}

Mais comment connaître l'efficacité de cette politique de valorisation des collections destinées à la consultation sur place ? Par ailleurs, sans contrôle d'accès, que savions-vous précisément de ces lecteurs qui viennent lire et consulter sur place? 


\section{LA CONSULTATION SUR PLACE}

Pour remédier à ces deux inconnues majeures, la bibliothèque de Sciences Po s'est depuis longtemps dotée d'outils d'enquêtes permettant de collecter des données d'usage relatives à la consultation. Ainsi dès 2007, en s'inspirant de ce qui se faisait déjà à la Bpi, elle a entamé une évaluation annuelle de la consultation via la « semaine test de la consultation ». Il restait à en savoir plus sur les lecteurs (ou plutôt les " consulteurs ») car construire une politique documentaire ne se conçoit qu'en fonction des publics-cibles, surtout dans le cas d'une BU où l'on sait que les besoins et les usages évoluent de façon importante entre les premier et deuxième cycles. C'est pour tenter de cerner cette deuxième inconnue que très récemment la bibliothèque a entrepris d'interroger et d'observer ses usagers pour connaître plus globalement leur rapport à la collection imprimée. En écho à cette démarche entreprise par la bibliothèque de Sciences Po, on peut se référer aux recommandations de la norme ISO $16439^{1}$.

\section{ENCADRÉ RECOMMANDATIONS DE LA NORME ISO 16439}

« La norme recommande de s'appuyer sur trois catégories de données selon les modalités de leur collecte :

- les données induites (inferred) des statistiques habituellement collectées par la bibliothèque dans le cadre de ses activités et suivies par la bibliothèque ;

- les données sollicitées (solicited) : il s'agit d'informations ou de données que l'on va collecter auprès des usagers au moyen de questionnaires ou d'entretiens ;

- les données observées (observed) qui sont le fruit d'observations dans le cadre de méthodologies inspirées de l'ethnologie $»^{2}$.

C'est ce panel de dispositifs que nous présentons ici, déployés afin de mieux cerner qui sont les usagers des salles de lecture, ce qu'ils y font précisément, comment ils s'y installent et quels sont leurs usages des collections en libre accès.

1. Livre blanc: Qu'est-ce qui fait la valeur des bibliothèques ?, Paris, Afnor/CN46-8 Qualité - Statistiques et évaluation des résultats, février 2016. [En ligne] : < http://portailgroupe.afnor.fr/ public_espacenormalisation/AFNORCN46-8/Livre\%20Blanc\%20fev2016.pdf >.

2. Ibid., p. 21. 


\section{QUI SONT LES USAGERS ET QUE FONT-ILS ? : DEUX ENQUÊTES}

Nous ne présenterons pas ici les enquêtes dans l'ordre chronologique où la bibliothèque de Sciences Po a entrepris de les mener (les semaines test de la consultation, dès 2007 ; Sweeping the Library en 2014 et « Que faitesvous en bibli aujourd'hui ? » en 2015), mais, dans un souci de logique, dans l'ordre de ce qu'il aurait fallu faire... Cependant, si cet ordre adopté empiriquement est à l'inverse de ce qu'il aurait fallu faire, dans la mesure où l'on s'est d'abord occupé des collections avant de s'intéresser aux publics, ce n'est que le reflet de l'évolution de l'évaluation en bibliothèque qui, dans les années 2000, s'est attachée à mesurer l'activité des collections, avant de se consacrer, dans les années 2010, aux publics.

Pour mieux connaître qui sont nos publics, nous avons entrepris deux types d'évaluation. L'une se range dans la catégorie des données « observées », l'autre, dans celle des données " sollicitées ». La première inspirée d'enquêtes menées à plusieurs reprises à l'étranger porte le nom de Sweeping the Library. La seconde est une enquête par questionnaire que l'on a appelé «Que faites-vous en bibli aujourd'hui ? »

\section{SWEEPING THE LIBRARY : CARTOGRAPHIER L'UTILISATION DES ESPACES ET DES OBJETS DE LA BIBLIOTHĖQUE}

L'objectif principal de cette méthodologie d'enquête est de cartographier l'organisation physique de la bibliothèque et l'utilisation des espaces au moyen d'une grille d'observation des profils, matériels et activités des usagers installés dans les différentes salles de lecture. À l'issue de près de neuf mois d'enquête et de 2407 observations collectées dans tous les espaces, à toutes les heures de tous les jours de la semaine, il a été possible d'extraire une batterie d'observations concernant à la fois :

- la répartition des usagers sur les dix plateaux de lecture ;

- leur préférence pour tel ou tel type de mobilier ;

- leur usage des postes informatiques mis à disposition par la bibliothèque, ou de leur propre équipement ; 
- l'usage (quasi permanent) de smartphone en parallèle aux travaux universitaires ;

- la présence de cahiers, stylo, livres et périodiques sur leur table de travail ;

- l'activité en cours.

Cette enquête ayant déjà été présentée par ailleurs ${ }^{3}$, nous ne reviendrons pas sur le détail des résultats. Ce qui nous intéresse ici c'est l'observation du rapport des usagers aux collections imprimées de la bibliothèque et la variété des informations que l'on a pu extraire de ces 2407 observations sur ce sujet précis.

Environ $28 \%$ des usagers observés avaient un livre ou un périodique sur leur table de travail. Parmi ces 659 personnes, 262 s'en servaient comme support de lecture, 183 comme support de prise de notes. Le nombre moyen de livres observés sur les tables de travail pour les 2407 observations est de 1,73. Ce chiffre monte à 2,12 pour ceux qui sont justement en train de le lire et à 2,1 pour ceux qui s'en servent en support de prise de notes.

Finalement, on a pu constater que les 659 usagers qui avaient des livres ou des périodiques étaient plus souvent que les autres investis dans une activité de prise de notes (163) ; étaient à la photocopieuse (15) ; étaient en train de lire (231) ; étaient présents les premières semaines de juillet (c'est le moment à Sciences Po où certains étudiants viennent préparer les concours) ; étaient au guichet prêt/retour.

On le voit, être vu avec un livre en bibliothèque signifie toute sorte de choses qui ne sont pas forcément liées à la consultation ou à la lecture immédiate de l'ouvrage en question!

Par ailleurs, pourquoi vouloir dénombrer à tout prix un nombre important de livres entre les mains des usagers ? Que veut la bibliothèque ? Que ses collections circulent et pouvoir afficher un grand nombre d'ouvrages en

3. Cette enquête et ses résultats ont déjà donné lieu à un article : Hélène Saada et Cécile Touitou, « Sweeping the Library : la bibliothèque de Sciences Po Paris passe les usages de ses lecteurs au tamis de l'observation », Bulletin des bibliothèques de France, juin 2015. [En ligne] : < http:// bbf.enssib.fr/contributions/sweeping-the-library >. 
circulation ou bien que les ouvrages soient lus et aient un impact positif sur les lecteurs (et contribuent à la réussite des étudiants) ?

C'est là encore une évolution de la notion d'évaluation qui est introduite dans la norme 16439 : veut-on savoir combien de documents ont été « déplacés » de leur emplacement ou bien souhaite-t-on mesurer le bénéfice que les lecteurs ont tiré de ces collections, notamment en BU, en mesurant l'impact de cet usage dans la réussite étudiante ? Cette mesure de l'impact voire de l'outcome est tout l'enjeu des évaluations qu'il conviendrait de mener. "L'outcome désigne l'ensemble des transformations ou des changements induits dans la vie, l'expérience ou la pratique de l'usager ou de la communauté en relation avec les missions d'éducation et de mise à disposition de la documentation des bibliothèques $»^{4}$. Pour y parvenir, il conviendrait d'interroger les usagers sur les bénéfices qu'ils ont tirés de la lecture de la documentation mise à disposition par la bibliothèque. Nous n'avons pas encore à la bibliothèque de Sciences Po mené ce type d'enquête, mais nous avons franchi une première étape en interrogeant les usagers sur les motivations de leur visite.

\section{« QUE FAITES-VOUS AUJOURD'HUI EN BIBLI ? »}

Sans contrôle d'accès, nous n'avions pas de visibilité sur qui était présent en salle. Inspirés d'une enquête qui avait été menée à la BU de l'université McGill à Montréal ${ }^{5}$, nous avons construit une enquête permettant de prendre une photographie des présents lors d'un après-midi et d'une matinée du mois d'octobre 2015, jour de grande affluence.

Un questionnaire était distribué systématiquement à toutes les personnes entrant dans la bibliothèque. Délibérément court, il avait pour ambition de permettre une meilleure connaissance de qui étaient les usagers ; quels étaient leurs motifs de visite ; quelle était la durée de leur séjour ; dans quel espace ils allaient s'installer.

4. Ibid., p. 10

5. McGill Library Visitor Survey: A one-day census to find out more about our users! [En ligne] : < http://blogs.library.mcgill.ca/channels/mcgill-library-visitor-survey-a-one-day-census-to-findout-more-about-our-users/ >. 


RÉPARTITION DES TROIS MOTIFS PRINCIPAUX
DE VISITE À LA BIBLIOTHĖQUE - POURCENTAGES
CALCULÉS SUR LA BASE DES RÉPONSES
POUR CHAQUE RANG $(1,2$ ET 3)

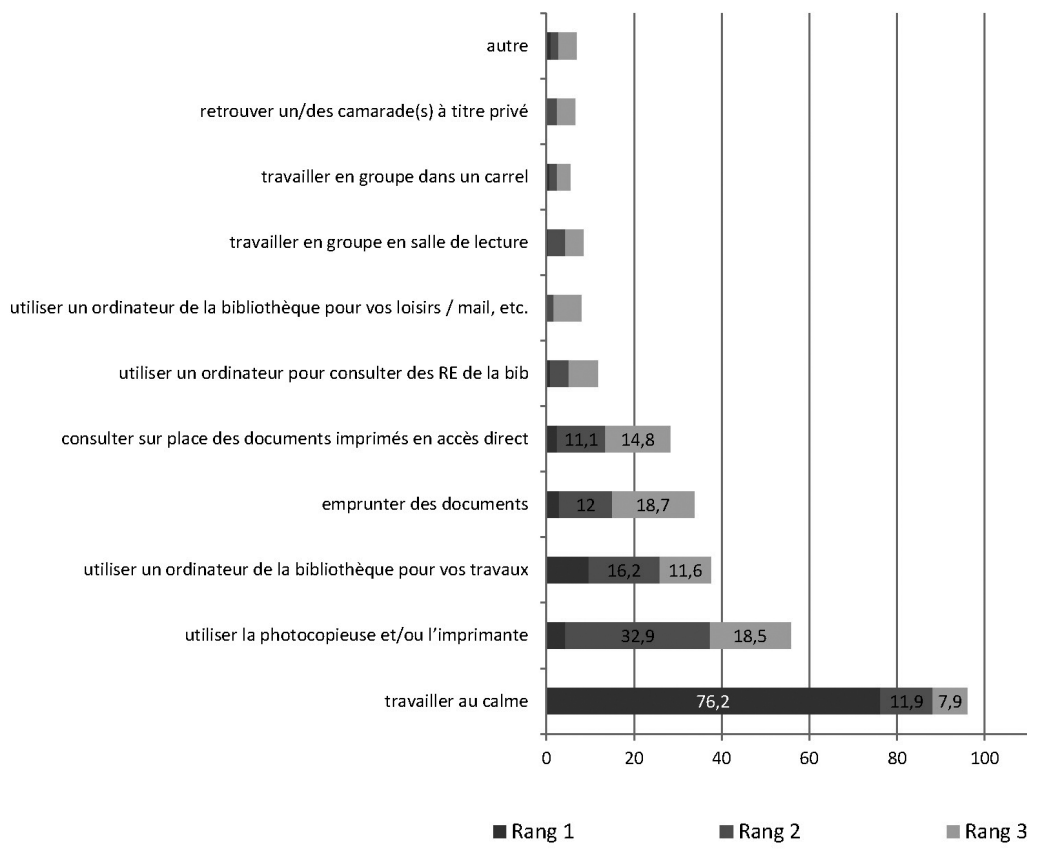

Source : Enquête Vous faites quoi en bibli ? Bibliothèque de Sciences Po Paris, 2015.

Après la saisie de 1220 réponses collectées, nous avons eu quelques surprises concernant la présence des étudiants en bibliothèque. Avec $55 \%$ de déclarants en $4^{\mathrm{e}}$ et $5^{\mathrm{e}}$ années d'étude (M1 et M2), nous avons constaté une légère surreprésentation des étudiants en Master par rapport à leur poids dans les effectifs de la scolarité. Ce qui est plus intéressant et qui

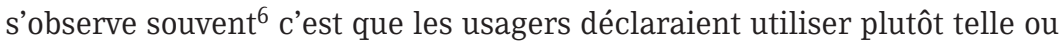

6. Philippe Galanopoulos, Les publics étudiants en $1^{\text {re }}$ année de médecine à la Bpi, Service études et recherche de la Bibliothèque publique d’information, 2010. [En ligne] : < http://www.bpi.fr/ observation-des-publics-de-la-bp/les-publics-etudiants-en-1ere-annee-de-medecine $>$. 
telle salle : les Masters s'installent plus souvent aux $4^{\mathrm{e}}$ et $5^{\mathrm{e}}$ étages de la bibliothèque du 27 rue-Saint-Guillaume, là où ils sont certains de trouver un peu de silence et là où ils pourront rester travailler longtemps (les usagers déclarent à $61 \%$ des séjours d'au moins une demi-journée au $5^{\mathrm{e}}$ étage versus $38 \%$ pour l'ensemble du bâtiment). Peu de mixité spontanée des publics (entre années d'étude) donc selon la règle bien connue en bibliothèque des publics qui se chassent l'un l'autre.

La dernière question, qui nous permettait de faire le lien avec d'autres enquêtes, où elle était également posée, est celle des raisons de la visite. Interrogés sur les rangs 1, 2 et 3 des motifs principaux de leur visite, c'est pour trois quarts d'entre eux "venir travailler au calme » qui est cité en rang 1 très loin devant tous les autres motifs suggérés (dans l'étude faite à McGill, c'était To study or get work done alone/étudier ou travailler tout seul qui venait en tête à $67 \%$ ).

\section{La consultation sur place}

Cependant, si la consultation sur place des documents n'est citée principalement qu'en rangs 2 et 3 des motifs, il ne faut pas pour autant conclure qu'elle est négligeable. La consultation est paradoxalement plus souvent citée par les étudiants du premier cycle (22\% d'entre eux) que par les étudiants du second (16\%). Les 272 personnes (sur 1220 ) qui l'ont mentionnée viennent plus souvent que le reste des répondants pour la journée entière et plus souvent dans la bibliothèque située 30 rue Saint-Guillaume qui abrite les collections en histoire, droit et science politique.

Nous voyons à la lumière de ces deux enquêtes à quel point la segmentation des publics en fonction de leur usage est importante pour mieux comprendre les besoins et les attentes variées de ces publics composites.

\section{MAIS S'ILS LISENT, CONSULTENT, FEUILLÈTENT, QUE REGARDENT-ILS EXACTEMENT ?}

La consultation sur place des documents n'est donc pas la raison principale et majoritaire de la visite en BU... Les étudiants " préfèrent » sans doute emprunter que consulter ou plutôt... les étudiants ont plus souvent 
l'occasion d'emprunter que de consulter, pratique limitée à un usage particulier de la documentation pour la révision et la vérification d’informations à condition de trouver une place assise à proximité des collections concernées dans une bibliothèque comprenant dix plateaux de lecture ! Mais que consultent-ils ? Là encore, la bibliothèque de Sciences Po a déployé un dispositif d'évaluation annuelle (et maintenant bisannuelle) permettant de nourrir la réflexion sur la politique documentaire et l'offre en salles de lecture. Dans un contexte de pénurie importante de rayonnages en salles de lecture ne permettant d'exposer en libre accès que $10 \%$ du fonds d'ouvrages disponibles (2 157 mètres linéaires versus 22144 mètres linéaires en magasin), il est impérieux de vérifier et d'évaluer le bon usage de ces ouvrages précautionneusement choisis.

Les semaines tests de la consultation ont été largement présentées par ailleurs ${ }^{7}$. Ici, nous aimerions succinctement décrire les données qu'il est possible de détenir à travers ce dispositif et en quoi il contribue à construire cette photographie complète des usages et des publics que nous tentons de réaliser.

Après avoir scanné tous les codes-barres des ouvrages consultés et laissés sur les chariots disposés en salle de lecture par les lecteurs, deux fois une semaine par an, il nous est possible d'extraire toutes les données utiles du SIGB se rapportant à la description bibliographique ainsi qu'aux exemplaires consultés. De cette masse d'informations, nous pouvons à la fois extraire une vision diachronique sur le volume des documents consultés et leur variation d'année en année, mais aussi nous pouvons disposer d'une analyse qualitative fine sur la qualité de ces documents, notamment concernant la répartition des consultations par :

- jour, heure, salle de lecture ;

- année d'édition ;

- langue du document ;

- domaine thématique (tranches de côtes).

7. Muriel Amar et Bruno Béguet, « Les semaines tests : évaluation de l'utilisation des collections imprimées sur place à la Bpi », Bulletin des bibliothèques de France, 2006, n 6, pp. 36-42. [En ligne] : < http://bbf.enssib.fr/consulter/bbf-2006-06-0036-007 >. 
Après plus de sept années d'observations, si nous déplorons la baisse effective du nombre de documents consultés (malgré une bibliothèque de plus en plus saturée, et de plus en plus ouverte, jusqu'à 85 heures annuelles), comme un peu partout dans les BU, nous observons des tendances que l'on pourrait considérer comme préoccupantes car elles s'apparentent à un " effet best-seller » :

- resserrement de la consultation sur des ouvrages de plus en plus récents (mais pas pour toutes les disciplines, l'histoire subissant « naturellement » moins cette tendance que le droit) ;

- resserrement de la consultation sur un nombre de domaines thématiques de plus en plus limité.

La semaine test enfin nous permet, au-delà des seuls résultats sur la volumétrie de la consultation qui présentent trop de biais, de rapporter la part de la consultation d'un domaine thématique, ou bien d'une langue, à la part de cette collection dans le total des collections offertes en libre accès. " Le taux de consultation évalue le volume des consultations qu'a connu un secteur (ou un domaine) relativement à l'ensemble des consultations d'une semaine test et à la totalité de l'offre documentaire imprimée, ce qui permet de gommer les effets dus aux écarts de volumes entre secteurs (ou domaines) : il résume les différences entre la structure des collections et celle des consultations en une seule valeur pondérée $»^{8}$.

Déployer et maintenir ce dispositif d'enquêtes d'année en année est un investissement lourd de la part de tous les collègues qui prennent en charge leur administration et leur analyse. Le partage de ce travail permet la diffusion de la " culture de l'évaluation » dans l'ensemble du personnel. La délicate prise en compte des résultats comme aide au pilotage peut alors continuer à orienter ou éclairer le pilotage stratégique. Pour ce faire, avoir constamment à l'esprit la mise en relation des données induites, sollicitées et observées favorise la construction d'une représentation fidèle des publics et de leurs usages, permettant d'adapter l'offre à la réalité de la demande, et fournit les éléments nécessaires à l'élaboration d'un plaidoyer pour la bibliothèque. 


\section{5}

LE TRI PAR CARTES POUR AMÉLIORER L'ARBORESCENCE DU Joris Paillaré SITE WEB DE LA BIBLIOTHĖQUE DE SCIENCES PO PARIS

En 2016, la bibliothèque de Sciences Po a expérimenté le tri par cartes dans le cadre d'un projet de refonte de son site web. Cette méthode, bien documentée sur Internet, contribue à optimiser l'architecture de l'information d'un site en faisant directement participer les usagers.

\section{ENCADRÉ LES TROIS TEMPS DU TRI PAR CARTES}

Le déroulé d'une session de tri par cartes est simple :

1. vous présentez à chaque participant des cartes en papier sur lesquelles vous avez inscrit des titres de contenus rédactionnels (un titre par carte) ;

2. chaque participant classe les cartes suivant sa propre logique ;

3. il nomme les regroupements ainsi formés.

\section{ASSIGNER UN OBJECTIF À CHAQUE TRI}

+++++++++++++++++++++++++++++++++++++++++++++++++++++++++++++++++++++++++++

Les utilisateurs ont tendance à organiser les cartes selon :

- leur niveau de connaissance des sujets ;

- leur intérêt pour ces sujets.

Ces paramètres sont cruciaux pour comprendre qu'on ne peut pas traiter tous les contenus d'un site par un seul tri.

Pour le projet Sciences Po Paris, l'objectif était de mener une première série de tris pour définir les grandes rubriques du site en ciblant en priorité les attentes des étudiants du campus parisien. 
Retenez qu'un tri permet de constituer des catégories de même niveau, pas toute la structure hiérarchique d'un site.

Aussi, pour organiser le contenu d'une rubrique particulière ou des contenus s'adressant à des publics spécifiques, il faut préparer des tris adaptés.

\section{PRÉPARATION}

La première étape consiste à remettre à plat les pages du site, c'est-à-dire à inventorier et décrire les contenus publiés, en précisant notamment les publics concernés. Ensuite, il faut choisir les contenus pertinents. C'est l'étape la plus délicate.

Pour notre projet, une vingtaine d'informations et services destinés aux étudiants ont été retenus avec deux règles de précaution :

- écarter des intitulés qui induisent des regroupements tels que « Services » ou « Pratique »;

- ne pas sélectionner des contenus reliés par des relations hiérarchiques (terme générique, terme spécifique).

Plusieurs types de tri existent : ouverts ou fermés, papier ou en ligne, présentiels ou à distance. Nous avons privilégié des tris :

- ouverts : chaque utilisateur définit et nomme ses propres catégories ;

- papier et en ligne : le premier permet aisément de modifier, ajouter, dupliquer des cartes. Les solutions en ligne de card sorting figent les contenus mais offrent de puissantes possibilités d'analyse quantitative ;

- présentiels : favorisent l'interaction et le recueil d'informations qualitatives.

Les études montrent que 15 participants permettent d'obtenir un bon rapport résultats/coût. Pour notre projet, 6 étudiants de $1^{\mathrm{er}}$ cycle ont été recrutés pour une première série de tris papier qui nous a permis de procéder 
à des ajustements pour préparer une seconde série de tris en ligne avec 16 étudiants de Master.

Lors du déroulement des tris, des collègues jouant le rôle de modérateurs ont répondu aux difficultés de compréhension, et incité les participants à verbaliser leurs choix tout en veillant à ne pas influencer les logiques de classement.

\section{ANALYSE ET RÉSULTATS}

Les résultats quantitatifs collectés ont été analysés avec des outils qui ont permis notamment :

- d'identifier les fréquences d'associations, autrement dit de montrer les contenus que les utilisateurs mettent ensemble ;

- d'aligner et consolider les catégories en clusters, facilitant ainsi l'analyse de la distribution des cartes. Très utiles lorsque l'on doit analyser une centaine de regroupements (chaque participant a créé en moyenne 6 catégories), ces clusters représentent les rubriques de premier niveau que nous souhaitions définir.

L'itération permet d'augmenter la qualité des résultats collectés.

Enfin, le tri par cartes se base sur une représentation mentale des utilisateurs, pas sur une mise en situation réelle de recherche d'information. Le design du système de navigation devra s'appuyer sur des techniques de conception et d'évaluation complémentaires, comme les tests d'utilisabilité1.

1. Voir la contribution de Nicolas Alarcon : «Évaluer l'utilisabilité des sites web », p. 150. 
6

ÉVALUER L'USAGE

DES RESSOURCES NUMÉRIQUES : QUELS ENJEUX, QUELLES SOLUTIONS?

par

Thomas

Jouneau

et Thomas

Porquet

Nous traitons ici de la question stratégique de la mesure des accès aux ressources numériques. Nous visons en particulier les établissements de l'enseignement supérieur et de la recherche (ESR), avec pour exemple l'utilisation à l'université de Lorraine (UL) d'ezPAARSE, un outil logiciel libre et gratuit qui permet de compléter et préciser les données d'usage, fournies par les éditeurs, par des données collectées et traitées localement. Ce moyen permet aux bibliothèques de regagner une certaine autonomie dans le domaine du traitement des données d'accès aux ressources souscrites.

L'augmentation constante des montants d'abonnements aux ressources numériques, dans un contexte de stagnation ou de baisse des budgets, est un fait connu et nous n'y reviendrons pas en détail ${ }^{1}$. On peut en revanche souligner la nécessité de disposer de données qui permettent de préciser un suivi et une vision stratégique pour la conduite d'une politique documentaire en ce qui concerne les abonnements, ou d'une manière générale, de mieux connaître leur appropriation par les communautés d'utilisateurs.

À travers le cas concret de l'UL, nous allons évoquer les différentes questions qui se sont posées au moment de la mise en œuvre d'ezPAARSE puis pendant son exploitation, ainsi que les réponses apportées.

1. Voir l'édition la plus récente du STM Report : < http ://www.stm-assoc.org/2015_02_20_STM_ Report_2015.pdf >. 


\section{POURQUOI ET COMMENT TRAVAILLER AVEC EZPAARSE ?}

\section{L'INTÉRÊT DU LOGICIEL POUR COMPLÉTER LES CHIFFRES ÉDITEURS}

Les établissements abonnés à des ressources numériques travaillent encore majoritairement avec des statistiques fournies par les éditeurs ${ }^{2}$. Dans le meilleur des cas, ces fichiers suivent Counter, une recommandation internationale devenue un standard de fait, et dont le Code of practice ${ }^{3}$ détaille la façon dont ces statistiques d'accès doivent être générées et présentées. Malheureusement, beaucoup d'éditeurs ne s'y conforment pas : soit en n'offrant aucune donnée, ou aucune garantie sur le mode de calcul, soit en proposant des modes de comptage (« crédits », " pages vues », etc.) ne pouvant être rapprochés des éléments couverts par Counter (articles, chapitres, etc.).

Les éditeurs respectant Counter livrent des données en théorie fiables, auditées et homogènes. Elles restent toutefois agrégées au niveau d'un compte (généralement un établissement) et n'offrent pas de visibilité sur l'usage des ressources par différentes catégories d'utilisateurs (discipline de spécialité, statut, niveau d'études...). A contrario, les données plus classiques de prêt et d'inscription, universellement exploitées, offrent ce genre d'information depuis les débuts de l'informatisation des bibliothèques. Il apparaît souhaitable de pouvoir disposer de ce même niveau de précision pour les ressources en ligne, qui représentent aujourd'hui, dans de nombreux établissements, un montant de dépenses supérieur à celui consacré au papier.

\section{LE PRINCIPE DE FONCTIONNEMENT : LE MATÉRIAU, CE QU'ON EN FAIT}

C'est ici qu'ezPAARSE entre en jeu. Basé sur le modèle fonctionnel d'une solution locale développée à l'INIST-CNRS en 2009 (NumStat), ezPAARSE est développé selon une méthodologie agile* pour l'ensemble de l'ESR, grâce à un partenariat organisé entre l'INIST-CNRS, Couperin.org et l'UL entre octobre 2012 et décembre 2015.

\footnotetext{
2. Voir à ce sujet l'enquête menée par le projet Counter sur ce thème : < http ://www.projectcounter.org/documents/COUNTER_Survey_Library_Results.pdf $>$.

3. Code of practice $:<$ http ://projectcounter.org/ $>$.
} 
Son mode de fonctionnement repose sur l'analyse des fichiers $\operatorname{logs}^{4}$ que collecte le reverse proxy ${ }^{5}$ mis en place pour l'accès authentifié aux ressources numériques souscrites. La technique de l'analyse de logs est bien décrite ${ }^{6}$ et sa faisabilité technique est établie, moyennant certains pré-requis : ezPAARSE ne peut travailler qu'en présence de fichiers logs. Si les accès à la documentation numérique ne transitent pas par un proxy, ezPAARSE ne pourra rien en dire. Il existe beaucoup d'établissements qui ne proxifient que les accès distants (hors les murs) de leurs personnels et étudiants. C'est un élément qu'il faudra prendre en compte au moment de l'adoption du logiciel, qui trouve son plein intérêt dans le cas d'une proxification complète des accès.

\section{ENCADRÉ PRÉSENTATION D'UNE LIGNE DE LOG STANDARD}

Voici le contenu d'une ligne de log standard pour un accès réussi à une ressource numérique :

66.130.77.181 [01/02/2016 $10: 23: 01+0200]$ 'GET http ://pdn.sciencedirect.com/science? pii=S00014575\&docid=1-s2.0-main.pdf HTTP/1.1’ 2002546

On y trouve dans l'ordre : l'adresse IP du poste de l'utilisateur ; la date précise de la requête (à la milliseconde près) ; la requête HTTP avec l'URL de la page consultée, le code retour HTTP 200 qui indique que l'accès a bien eu lieu, et enfin la taille de la réponse en octets.

Toutes les lignes de logs présentes dans un fichier de logs (qui peut en contenir des millions) sont formatées de la même façon. On travaille avec un matériau homogène qui fournit des informations à la fois sur

4. Un log désigne les traces enregistrées dans un fichier ou tout autre dispositif, comportant l'historique horodaté des événements attachés à un processus.

5. $\mathrm{Ou}$ « serveur mandataire inverse ». Les plus connus sont EZProxy et BiblioPAM, mais d'autres peuvent aussi être utilisés comme Squid, Apache, etc.

6. Lisa Goddard, "Getting to the Source: A Survey of Quantitative Data Sources Available to the Everyday Librarian: Part I: Web Server Log Analysis" et "Part II: Data Sources from Specific Library Applications", Evidence Based Library and Information Practice, 2007, vol. 2, $\mathrm{n}^{\circ} 1$, pp. 48-88. [En ligne] : < https ://ejournals.library.ualberta.ca/index.php/EBLIP/article/ view/196/240 >. 
l'utilisateur (son adresse $\mathrm{IP}^{7}$, parfois son login et des attributs $\mathrm{LDAP}^{8}$ ) et sur la ressource consultée (l'URL et la taille de la réponse).

Après un travail de filtrage de toutes les lignes qui ne sont pas significatives ${ }^{9}$ en termes d'accès à une ressource documentaire, ezPAARSE génère des événements de consultation (EC) qui contiennent des enrichissements de type bibliographique (titre de la revue, ISSN, DOI, etc.), grâce au parseur ${ }^{10}$ qui correspond à la plate-forme sur laquelle la ressource est consultée. Les informations d'origine sont naturellement conservées.

Suite à ce processus, les fichiers produits sont de taille bien plus modeste que les logs (environ 10 fois plus petits) et ne posent pas de problème particulier de stockage : ainsi à l'UL, université dotée de 52000 étudiants et 8000 enseignants et chercheurs, on obtient des sorties allant, selon le mois concerné, de 120000 à plus de 200000 EC, chacune correspondant à un fichier compressé de 11 à 16 Mo.

\section{DE L'UTILISATION MANUELLE SUR L'INSTANCE NATIONALE À L'INSTALLATION LOCALE AUTOMATISÉE}

L'adoption d'ezPAARSE se fait progressivement et en trois étapes :

\section{Première étape : test du logiciel}

La phase de test se déroule soit sur l'instance nationale ${ }^{11}$, soit en local après installation sur un poste équipé d'un système d'exploitation de type Linux. Définir son format de log est crucial : c'est ce qui permet à ezPAARSE de reconnaître les logs pour les traiter. Si le format n'est pas reconnu par défaut, un outil intégré est proposé. Cette opération n'est pas complexe,

7. Une adresse IP (pour Internet Protocol) est un numéro unique permettant à un ordinateur de communiquer dans un réseau.

8. On désigne ainsi les caractéristiques qui définissent les entrées de l'annuaire LDAP (pour Lightweight Directory Access Protocol).

9. Entrent dans cette catégorie : la récupération des feuilles de style, les images, les fichiers javascript, etc. par le navigateur de l'utilisateur pour générer la page web complète ainsi que tous les accès non réussis à des ressources et les redirections.

10. Dans ce contexte, le parseur est l'analyseur syntaxique destiné à récupérer les informations contenues dans les URL d'accès aux ressources.

11. [En ligne] : <http ://ezpaarse.couperin.org >. 
mais utilise un formalisme (les expressions régulières) qui n'est pas nécessairement connu de tous.

EzPAARSE est alors en mesure de livrer ses premiers résultats. Il faut vérifier que les informations permettant de caractériser les catégories d'utilisateurs sont présentes : soit en paramétrant son reverse proxy de façon à ce qu'il ait accès à la donnée de catégorie directement, soit en procédant à un enrichissement a posteriori, à l'aide par exemple d'un script de rapatriement d'attributs LDAP.

Les fichiers de rejets fournis par ezPAARSE sont utilisés pour évaluer le taux de couverture en ressources reconnues en comparant le nombre de ressources auxquelles un établissement est abonné et le nombre de plates-formes pour lesquelles on souhaite obtenir des données d'accès caractérisées. EzPAARSE est actuellement livré avec une centaine de parseurs qui couvrent la grande majorité des grands éditeurs et plates-formes de diffusion. Le site Analogist-NG ${ }^{12}$ recense les plates-formes couvertes ou en passe de l'être.

\section{Deuxième étape : constitution d'un dossier pour adoption}

Ce premier test conclu (le format de log est reconnu ; la couverture par ezPAARSE des abonnements semble suffisante), l'application et ses résultats peuvent être présentés à une équipe, à une direction des systèmes d'information (DSI), à une direction. Un plan d'action collectif pour la mise en production du logiciel et son exploitation sera alors établi. Une étape à conduire en parallèle et à ne pas négliger est celle du rendez-vous avec le correspondant Informatique et Libertés (CIL) de l'établissement. Par défaut, ezPAARSE fait un usage intensif de données à caractère personnel et nécessite d'en déclarer l'usage, de définir les conditions et la durée de conservation adéquates des fichiers de logs et des résultats obtenus ${ }^{13}$.

12. Analogist-NG : < http ://ang.couperin.org >.

13. Voir les fiches du Guide pratique Informatique et Libertés, co-édité par la Conférences des présidents d'université (CPU), la Commission nationale de l'informatique et des libertés (CNIL) et l'Agence de mutualisation des universités et établissements (Amue), en particulier la fiche 13 : « Contrôle de l'utilisation des moyens informatiques ». 


\section{Troisième étape : mise en production}

La DSI est désormais impliquée et s'occupe d'installer le logiciel sur un serveur. Le rythme de traitement (généralement mensuel) auquel les logs sont ingérés ainsi que les emplacements auxquels les logs sont récupérés et les résultats livrés seront ensuite définis. L'expérience de l'équipe ezPAARSE et des établissements déjà utilisateurs peut ici aider à définir une solution adaptée pour un établissement ${ }^{14}$.

\section{L'EXPLOITATION DU LOGICIEL : LE CAS DE L'UL}

\section{L'UL EN UTILISATEUR PILOTE}

L'UL est née en 2012 de la fusion de quatre établissements (Nancy-1, Nancy2, Institut national polytechnique de Lorraine, Metz). Suite à un travail commun des différents services de documentation, un reverse proxy commun est adopté dès 2009 (EZproxy) et gère les accès à la documentation numérique payée, tant en local qu'à distance. Ce choix est en contraste avec celui de certains autres établissements, qui réservent l'usage des serveurs mandataires à l'accès distant. Il a nécessité, dans certains cas, une adaptation des usages ${ }^{15}$. Entre autres démarches d'accompagnement, un bookmarklet ${ }^{16}$ est proposé aux lecteurs pour transformer automatiquement les URL. Ce passage de l'ensemble des accès par le reverse proxy est toutefois une condition cruciale si l'on veut pouvoir tirer de l'exploitation des logs par ezPAARSE des jeux de données complets.

Autre acquis de la fusion : la présence d'un annuaire unifié, brique importante pour l'exploitation ultérieure des résultats. Deux éléments se sont avérés particulièrement importants : les affiliations, qui obéissent à des règles d'écriture strictes et documentées; les catégories d'usagers ou Business Categories (BC), au nombre d'une vingtaine, sont utilisées pour gérer une partie des droits à l'ensemble des services numériques de l'UL, ressources documentaires incluses. Ces deux éléments étaient remontés

14. Il existe une liste de diffusion : < https://groupes.renater.fr/sympa/info/ezpaarse-users >.

15. L'accès aux ressources par un reverse proxy exige une transformation des URL.

16. Petit programme JavaScript pouvant être stocké en tant qu'URL dans un favori ou un hyperlien dans une page web. 
dans les logs avant même le début de la collaboration autour d'ezPAARSE, et sont amplement utilisés dans l'exploitation. Le passage exclusif par le reverse proxy et la proximité géographique de l'UL avec l'INIST-CNRS, ont favorisé le positionnement de l'établissement comme pilote pour le déploiement du logiciel.

L'installation et l'exploitation du logiciel se sont faites au départ avec une implication humaine très réduite, soit deux personnes, membres de l'équipe ezPAARSE : un informaticien (également administrateur d'EZproxy) et un bibliothécaire, tous deux à temps très partiel sur ce travail. Cette première installation sommaire a fait place début 2015 à une installation automatisée avec serveur dédié pour les fichiers résultats produits.

\section{PRODUCTION ET EXPLOITATION DES DONNÉES}

À l'UL, les sorties ezPAARSE sont augmentées de trois éléments de données dans chaque événement de consultation. Aux BC et affiliations, déjà citées, s'est ajouté plus récemment le code ETAPE, issu d'une nomenclature nationale des cursus, diplômes et niveaux, pour obtenir des statistiques plus fines que ne le permettent les deux autres éléments, en caractérisant le niveau et la discipline du consultant. Sur la base de ces données, des renseignements plus précis (affiliations, cursus ou statuts en clair) sont rapatriés depuis les référentiels utilisés.

EzPAARSE ne représente en effet que l'une des étapes d'une chaîne de travail. Une fois obtenue une liste d'événements de consultation, il reste en effet deux étapes : l'une facultative, de post-traitement des fichiers, afin de procéder à des enrichissements et des manipulations qui n'auraient pas été automatisées en aval ; l'autre, plus importante, de consolidation et de visualisation des résultats. Il existe de nombreux outils permettant d'effectuer l'une ou l'autre tâche, ou les deux, du simple tableur à la suite avancée ${ }^{17}$. Le choix de l'UL s'est porté sur le logiciel Omniscope, de la société Visokio, déjà utilisé à l'INIST-CNRS, qui propose à la fois un

17. Un outil d'analyse sous la forme de fichier tableur avec macros est fourni en formats Excel et LibreOffice. Un module développé à l'INIST, ezVis, offre une visualisation interactive des résultats. Des logiciels comme Business Object ou QlikView couvrent chacun de ces deux aspects de la chaîne de travail. 
module de traitement de données ingénieux et un module de visualisation un peu rigide mais efficace.

Reprenons les données récupérées : de nombreux usagers des ressources numériques possèdent plusieurs statuts et affiliations en fonction de leurs différentes " casquettes » professionnelles ou étudiantes. Concernant le statut, c'est la BC principale (enseignant-chercheur, étudiant) qui est retenue. Concernant les affiliations, le problème est autre. Les enseignants-chercheurs, par exemple, possèdent au moins deux affiliations non hiérarchisées (composante d'enseignement et structure de recherche). La structuration des codes utilisés à l'UL permet toutefois de faire le tri, et c'est la première affiliation de chaque type parmi trois (recherche, formation, administration) qui est retenue. Il est en revanche difficile de construire des tableaux de bord classiques à deux dimensions en tenant compte des affiliations multiples : aussi, recherche et formation sont-elles traitées séparément. Une consultation par un enseignant-chercheur apparaîtra ainsi à la fois dans les tableaux de bord orientés recherche (avec laboratoire d'appartenance) et formation (assortie de la composante d'enseignement). Omniscope permet de créer des tableaux de bord dynamiques dont le destinataire contrôle certains éléments. Ainsi un premier tableau, orienté recherche, permet d'afficher des graphes d'utilisation par pôle de recherche et par laboratoire, et permet de ne sélectionner que l'un, ou plusieurs, de ces regroupements et unités. Un second, orienté formation, fait la même chose sur les collegiums et les composantes.

La pratique de ces tableaux vient dans un premier temps confirmer certains faits observables, comme la proportion très importante de consultations dans les composantes santé, sciences ou sciences juridiques. Des surprises sont présentes, comme la part des consultations étudiantes sur certaines ressources, qui monte de 25 à $33 \%$ des consultations sur les grandes platesformes académiques anglophones (ScienceDirect, Wiley...), et s'avère écrasante sur les plates-formes juridiques (LexisNexis, Lamyline...).

Dans un second temps, ces tableaux ouvrent des perspectives intéressantes en matière de pilotage. Les données de consultation des différentes unités et composantes peuvent déjà être utilisées par le service formation des bibliothèques pour cibler celles dont l'usage des ressources documentaires est en dessous de ce qui est attendu. Le service d'appui à la recherche 
récemment mis en place par les bibliothèques, avec des bibliothécaires référents par laboratoire, peut s'appuyer sur les profils d'usages des unités de recherche pour personnaliser au mieux les services proposés. Les décisions de politique documentaire numérique (abonnements et désabonnements) peuvent désormais être informées par une connaissance précise de leur utilisation par les communautés ciblées. Il reste, par contre, à combiner les données ezPAARSE avec celles plus classiques issues du SIGB ou des autres dispositifs professionnels, dans des tableaux de bord commun. Rien n'est parfait : beaucoup de ressources ne seront pas couvertes avant longtemps par ezPAARSE, faute d'URL analysables ; la mise à jour des parseurs est un travail réclamant vigilance et disponibilité, et qui peut engendrer des manques dans les tableaux de bord. L'adjonction de ces données à celles déjà disponibles via les rapports éditeurs permet toutefois d'atteindre un degré de connaissance de l'usage comme jamais précédemment.

\section{PERSPECTIVES}

Au-delà de l'intérêt immédiat de pouvoir générer des événements de consultation précis et enrichis pour un établissement, l'adoption du logiciel inscrit ses utilisateurs dans la communauté d'utilisateurs internationaux AnalogIST. C'est une porte ouverte pour accueillir les contributions aux analyses de nouvelles plates-formes éditeurs qui améliorent la couverture d'ezPAARSE et qui profitent automatiquement à toutes les autres instances installées : un mécanisme de mise à jour et de récupération des nouvelles plates-formes est embarqué dans ezPAARSE.

Le logiciel, dont le cycle de développement s'est achevé à la fin de l'année 2015 après 3 ans de travail, est, à la date de rédaction de la présente contribution, avril 2016, mis à disposition sous licence CeCILL ${ }^{18}$ dans une version stabilisée. Toutefois, pour assurer sa maintenance technique et continuer de le faire vivre, l'équipe constituée autour d'ezPAARSE organise la mise à contribution du logiciel dans d'autres projets. En voici deux exemples :

18. Adaptation en droit français de la GNU Public Licence. [En ligne] : < http://www.cecill.info/ licences/Licence_CeCILL_V2.1-fr.html >. 
- ezPAARSE comme source de données dans Agimus-NG. Agimus-NG est un projet de plate-forme dédiée à l'analyse des logs des applicatifs des établissements (messageries, espaces numériques de travail) sur un mode assez proche d'ezPAARSE ${ }^{19}$. Il s'agit de faire entrer les indicateurs de la documentation numérique dans les tableaux de bord dynamiques sur l'utilisation des services numériques à l'échelle de l'établissement et d'acquérir une visibilité sur ces questions, à la fois au sein de la DSI et de l'équipe dirigeante ;

- ezPAARSE comme brique de contribution à ezMESURE ${ }^{20}$, un projet de dépôt national de données produites par les instances ezPAARSE installées dans les établissements : ce projet (prévu sur 2 ans) vise à mettre en place un " agrégateur » national des statistiques locales produites par les différentes instances d'ezPAARSE installées dans les établissements et proposer une interface de consultation, en ligne et dynamique, consolidée et comparative, des données collectées.

19. « AGIMUS-NG permet d'utiliser les fichiers de traces [...] afin de produire un entrepôt de données [et] des indicateurs statistiques d'usage des services numériques. La plate-forme ingère des logs, les enrichit avec des informations du SI [...] et permet de créer des tableaux de bord. ». [En ligne] : < https ://www.esup-portail.org/wiki/display/AGIMUSNG/Documentations >. 


\begin{tabular}{|l|l|}
\multicolumn{2}{|c}{7} \\
\hline ÉVALUER L'UTILISABILITÉ & par \\
DES SITES WEB & Nicolas \\
\hline
\end{tabular}

En poste au bureau de renseignement, vous apercevez un usager en difficulté face à votre catalogue en ligne. Vous décidez de lui porter secours.

En le questionnant sur ses objectifs, vous comprenez qu'il cherche simplement à localiser un document dans vos locaux.

Il effectue à nouveau sa recherche devant vous en prenant soin de commenter le chemin emprunté et les liens cliqués, vous signifiant sa frustration d'arriver à une nouvelle impasse.

Votre oil d'expert vous permet d'identifier rapidement les nombreux problèmes dans la démarche de l'usager. Il n'a pas cliqué sur le bon lien, n'a pas saisi le sens du message affiché, qui paraît pourtant évident, et n'a pas su revenir sur la liste des résultats.

Si vous avez déjà vécu une telle situation (et vous êtes probablement nombreux dans ce cas) : félicitations, vous vous êtes confronté à la notion d'utilisabilité et vous avez même réalisé, sans le savoir, votre premier test d'utilisabilité.

L'utilisabilité représente le niveau de facilité, d'efficience, d'efficacité et de satisfaction auquel un utilisateur emploie un produit pour réaliser une activité précise.

\section{LES TESTS D'UTILISABILITÉ}

Les tests d'utilisabilité visent à mesurer ce niveau en enregistrant ou en observant la manière dont de vrais usagers réalisent des actions spécifiques sur ce produit. Cette méthode s'accompagne généralement d'une démarche de pensée à voix haute, où l'on demande au participant de verbaliser ses pensées tout en réalisant une tâche. Ces données sont ensuite analysées d'un point de vue quantitatif et qualitatif pour faire ressortir les problèmes rencontrés au quotidien par les utilisateurs.

Le produit en question peut aussi bien être un objet de la vie quotidienne qu'un service physique ou un site web. Pour une bibliothèque, le concept 
d'utilisabilité est d'abord appliqué aux outils numériques (site web, catalogue en ligne, bibliothèque virtuelle) mais il peut être aussi utilisé pour améliorer les espaces et les services in situ.

Pour être utilisable, un produit doit notamment répondre au critère d'efficience. Les moyens mis en œuvre dans son utilisation, mesurés par le nombre de clics ou la durée de navigation pour un site web, doivent être raisonnables au regard du résultat atteint. Le critère d'efficacité correspond au fait d'atteindre l'objectif qu'on s'était fixé en utilisant le produit. L'utilisabilité est primordiale dans l'expérience que se construira l'utilisateur face à un service ou un site web. Pourtant, l'utilisabilité ne recouvre pas complètement le concept d'expérience utilisateur (UX en anglais) qui représente " les réponses et perceptions d'une personne résultant de l'usage ou de l'anticipation de l'usage d'un produit, service ou système » (définition de la norme ISO 9241-210). L'expérience utilisateur est au carrefour de plusieurs faisceaux : utilité, navigabilité, accessibilité, désirabilité, crédibilité, valeur et... utilisabilité.

Dans notre exemple d'introduction, l'inefficacité du catalogue et la frustration qui en découle auront à coup sûr un impact négatif sur la perception que l'usager se fera de notre outil de recherche. Mauvaise impression de départ souvent très difficile à corriger.

\section{ENCADRÉ AMÉLIORER L'UTILISABILITÉ DU SITE : QUATRE BÉNÉFICES PRINCIPAUX}

Améliorer l'utilisabilité ne signifie pas simplement limiter ou supprimer la frustration des usagers. Cela servira aussi à :

- réduire les coûts de maintenance du site web. Dès la conception ou la refonte d'un site, un soin particulier porté à son utilisabilité lui garantira une meilleure espérance de vie ;

- diminuer les questions aux postes de renseignement. Une part non négligeable des questions aux postes d'accueil relève d'une utilisation biaisée du catalogue ou du site web ;

- éradiquer les formations aux outils numériques. Si votre site nécessite une formation ou un tutoriel pour correctement s'en servir, c'est qu'il doit être revu de fond en comble ; 
- assurer un certain niveau de trafic web sur vos outils en améliorant l'expérience utilisateur. Un usager qui trouve rapidement et sans effort ce qu'il cherche est un usager qui réutilisera vos outils.

L'avantage de l'utilisabilité est qu'elle constitue un concept assez souple pour être employé dans toutes les bibliothèques, des plus modestes à petit budget aux plus fastueuses et sans limite financière.

Les tests d'utilisabilité s'adapteront facilement à votre environnement, à votre calendrier et à vos publics. En décrivant les étapes de la création d'un test, les paragraphes suivants ont pour objectif de vous en convaincre.

\section{CRÉER UN TEST D'UTILISABILITÉ}

\section{LES SIX ÉTAPES}

\section{objectifs et scénarios}

La première étape est sans doute la plus frustrante car c'est celle des choix. Vous devrez laisser de côté d'innombrables questions que vous vous posez sur l'utilisation de vos outils numériques pour ne retenir que celles, prioritaires, qui seront l'objet du test.

Pour faire des choix, il faut évidemment bien connaître ses outils, avoir une idée de ce qui pose problème. Les intitulés des catégories sont-ils suffisamment compréhensibles ? Le graphisme ne fait-il pas trop daté, brouillant la navigation ? Ou est-ce plutôt l'architecture de l'information qui ne représente plus correctement la philosophie de votre institution? Pour défricher le terrain, il est possible de construire une enquête qualitative basée sur des focus groups et des entretiens individuels. Ces méthodes sont très fiables mais chronophages et souvent coûteuses. Une autre méthode pré-test d'utilisabilité est de rester attentif aux réactions et remarques des utilisateurs, de flâner régulièrement près des catalogues en ligne, d'observer leurs manières d'utiliser vos outils. Leur attitude corporelle vous indiquera le résultat de leur utilisation (agacement, découragement, abandon). Vos collègues constituent aussi une source non négligeable d'expériences à recueillir. 
En parallèle, dépouillez les statistiques d'usage de vos outils. Vous pourrez déterminer exactement ce que font les utilisateurs sur vos sites, par où ils arrivent, les pages qu'ils visitent, le temps passé et leur page de sortie. Autant d'éléments vous permettant de réaliser un premier classement des pages les plus consultées donc, a priori, celles sur lesquelles une attention supplémentaire devra être portée en matière d'expérience utilisateur. Une fois déterminés les éléments à étudier, il est nécessaire de préciser les objectifs attendus sous forme d'affirmation accompagnée d'indicateurs quantitatifs. Par exemple : "l'utilisateur sera capable de trouver la bonne icône en moins de 45 secondes et en moins de 2 erreurs ». Ces indicateurs seront calculés grâce à des tests blancs auprès d'utilisateurs novices et experts mais aussi en fonction des attentes vis-à-vis de la fonction testée. Ils représenteront un point de comparaison à partir duquel juger les résultats des utilisateurs.

Afin d'être compréhensibles lors des tests, ces objectifs seront transformés en scénarios, privilégiant une mise en situation réaliste pour les testeurs. Il conviendra de rester concis, d'éviter le jargon professionnel et de proposer assez d'informations pour effectuer la tâche assignée.

\section{Logistique et équipe}

$\mathrm{Du}$ point de vue matériel, les tests d'utilisabilité n'impliquent pas de se sur-équiper. Il suffit d'un ordinateur muni des périphériques de base, connecté à Internet et faisant tourner un système d'exploitation grand public pour réaliser un test. Le but est de proposer aux testeurs un environnement numérique familier qui ne parasitera pas les exercices demandés. Optez pour une salle calme de votre bibliothèque sauf si, plus fortuné, vous choisissez d'aménager une salle de test professionnelle avec miroir sans tain et moniteurs de retour.

La durée totale de l'enquête dépendra de plusieurs facteurs : complexité du produit à étudier, disponibilité et nombre de participants mobilisables, enregistrement audio/vidéo, etc. Une batterie de tests peut se gérer en 2 à 4 semaines pour les cas les plus simples.

L'enregistrement audio/vidéo de ce qui se passe à l'écran lors d'un test ne revêt jamais un caractère obligatoire. Ce choix sera déterminé par votre exigence et vos moyens en matière de qualité des données recueillies. 
Si vous n'enregistrez pas l'écran, cela signifie que vous devrez assister à l'exercice aux côtés de l'usager ou via un moniteur supplémentaire. Dans ce cas, vous ne pourrez pas revenir sur le déroulé des opérations pour analyser une séquence particulière, vous devrez faire confiance à vos notes et à votre mémoire, pas évident lorsque beaucoup d'informations pertinentes sont produites simultanément, mais pas impossible non plus. Si vous choisissez d'enregistrer l'écran, il existe de nombreuses applications libres ou propriétaires aux fonctionnalités plus ou moins avancées. Veillez néanmoins à ce qu'elles vous permettent d'enregistrer l'écran, de chronométrer les actions, de capturer la voix du participant, voire d'exporter images et sons sur d'autres logiciels.

La taille de l'équipe chargée de l'administration des tests variera selon les ressources humaines disponibles dans votre établissement. Il est possible de gérer l'ensemble du processus avec une seule personne même si cela reste compliqué. L'investissement en temps, de la conception à la diffusion des résultats, sera conséquent pour un agent qui cumule différentes fonctions (concepteur, recruteur, responsable logistique, modérateur, analyste, etc.) et donc plus susceptible d'oubli ou d'erreur.

L'idéal est de constituer une équipe restreinte, entre 2 et 4 personnes motivées, avec une répartition stricte des rôles et une coordination efficace entre les différentes tâches.

\section{Recrutement des testeurs}

Contrairement aux autres méthodes d'enquête, les tests d'utilisabilité ont l'immense avantage de ne pas baser leur fiabilité sur la taille et la composition d'échantillons de répondants comme pour les sondages. Il est admis que $80 \%$ des problèmes d'utilisabilité les plus importants seront décelés par 3 à 5 testeurs. Le retour sur investissement est donc comparativement très rapide.

Le bon nombre se situe entre 6 et 12 participants. Au-delà de 12, la probabilité de découvrir un nouveau problème est marginale. Ces éléments s'adaptent évidemment à la diversité de la population étudiée, au budget et aux échéances à respecter.

Le choix des utilisateurs sera effectué en fonction des niveaux d'expérience avec l'outil considéré (dans le cadre d'une bibliothèque : expérience 
d'Internet, des moteurs de recherche généralistes ou documentaires). Le nombre de caractéristiques pertinentes sera limité afin de ne pas multiplier les sous-groupes.

Un recrutement quelques semaines avant le test combiné à une confirmation immédiate et des rappels réguliers jusqu'à 1 ou 2 jours de l'échéance suffisent pour une bonne mobilisation des testeurs. Un taux d'absence de $10 \%$ n'est pas anormal. Pour encourager les participants à venir, le test doit être présenté comme un moment intéressant et convivial. Vous pouvez également envisager de rétribuer modestement les testeurs.

De la même manière que lancer un test d'utilisabilité nécessitait de bien connaître le produit, un bon recrutement ne peut se faire sans connaître vos publics : caractéristiques sociodémographiques, usages numériques, profils des non-fréquentants, etc. Ces données assureront un recrutement adapté à votre situation.

\section{comment mesurer l'utilisabilité ?}

Pour un site web, les mesures objectives à caractère quantitatif qui vous serviront à déterminer le degré d'utilisabilité de votre outil sont en premier lieu la réussite ou non de la tâche confiée, la durée de réalisation et le nombre de clics. Il est également possible de mesurer le nombre de mauvais choix ou le nombre d'erreurs similaires.

De plus, les mesures subjectives peuvent être de deux ordres :

- quantitative : pour la notation que vous demanderez aux testeurs d'appliquer en fin de séance afin de mesurer la facilité d'un exercice ;

- qualitative : pour les données généralement produites par la procédure de "pensée à voix haute ». Elles vous renseigneront sur le niveau d'aisance ou d'inconfort de l'utilisateur, sur ses moments d'impatience, de confusion, d'agacement ou d'enthousiasme.

Chaque mesure de performance sélectionnée pour votre test devra être estimée avec un triple niveau : 
- un niveau Excellent qui correspond à la mesure réalisée par une personne connaissant bien l'outil, bibliothécaire par exemple ;

- un niveau Correct c'est-à-dire une mesure que l'on est en droit d'attendre pour un usager lambda qui équivaut à une utilisabilité moyenne ;

- un niveau Inacceptable qui ne répond pas du tout au minimum d'utilisabilité requis.

N'oubliez pas que le temps des usagers est précieux, il ne faut pas en abuser surtout s'il n'y a pas de contrepartie pour eux. La durée d'un test ne peut donc excéder $1 \mathrm{~h} 30$.

Les exercices peuvent être limités dans le temps ( Si vous ne réussissez pas en moins de 3 minutes, passez à l'exercice suivant ») mais cela renforce le côté examen pour le testeur et fausse le réalisme de la procédure. La manière de réussir une tâche est plus importante que la durée de réalisation. Pour tirer parti de la concentration maximale des testeurs, placez les exercices les plus importants en début de séance. L'attention s'émoussant au fil du test, les derniers exercices pourraient être bâclés plus ou moins consciemment.

\section{Faire passer les tests}

Tout est prêt pour administrer les tests. Avant de vous lancer, pensez à réaliser le test à blanc au moins une fois avant le lancement officiel afin de déterminer la validité des objectifs, le bon fonctionnement du matériel en conditions réelles, la pertinence des mesures d'utilisabilité retenues, la durée totale du test et la clarté des scénarios.

Le modérateur devra rester attentif à ce que le test se déroule dans les meilleures conditions. Il n'hésitera pas à relancer amicalement le testeur s'il estime qu'il ne s'exprime pas assez à voix haute. Il prendra un moment après chaque test pour noter ses commentaires à chaud : déroulement global du test, comportement du testeur, difficulté ou problème rencontré, etc. Le test terminé, faites remplir au participant les questionnaires post-test, remerciez-le de sa venue, remettez-lui le lot d'incitation le cas échéant et profitez-en pour lui demander si vous pouvez conserver son adresse pour 
de futures enquêtes. Il est toujours intéressant de disposer d'une équipe restreinte de testeurs mobilisables rapidement.

\section{Analyser les données}

Les données produites doivent maintenant être analysées.

À l'aide d'un tableur ou d'une application plus sophistiquée, compilez les mesures quantitatives et calculez la moyenne, médiane, valeur limite pour chaque indicateur. Vous pourrez ensuite comparer ces données par sousgroupe d'utilisateurs en fonction des critères de sélection choisis lors du recrutement.

Les enregistrements audio et vidéo vous aideront à repérer les moments significatifs (enthousiasme, découragement, impatience, effet wow ${ }^{1}$, frustration) et le cheminement précis des testeurs durant les exercices. Il sera aisé de remarquer les problèmes d'utilisabilité, d'intitulé ou de navigabilité et d'y remédier.

\section{NI PANACÉE, NI CONCLUSION}

Les tests d'utilisabilité ne pourront constituer l'unique méthode de production de données dans vos projets. Car ils disent peu de chose sur qui sont vos publics, contrairement aux enquêtes de publics ou à la méthode des personas ${ }^{2}$. Car ils ne vous renseigneront pas sur les représentations des utilisateurs, préférez les focus groups ou les entretiens pour cela. Car ils sont moins pertinents que les tests $\mathrm{A} / \mathrm{B}^{3}$ quand il s'agit de choisir entre plusieurs versions d'un même produit.

Enfin, les tests d'utilisabilité s'insèrent nécessairement dans un processus itératif : design du projet, prototypage du produit, évaluations qui modifieront le design du projet et ainsi de suite.

1. Le marketing anglo-saxon designe par wow effect la capacité d'un produit ou d'un service à surprendre, étonner, émerveiller. On a pu parler de " marketing de l'enchantement ».

2. Les personas sont des archétypes d'utilisateurs possibles de l'application développée. Les concepteurs pourront s'y référer lors de la conception de l'interface afin de prévoir tous les usages et attentes possibles.

3. Le test $\mathrm{A} / \mathrm{B}$ est une méthode permettant de soumettre des versions légèrement différentes d'un même outil afin d'évaluer leur réception par des groupes distincts d'usagers et choisir la meilleure. 
Si la recherche constante d'amélioration grâce aux retours des utilisateurs ne garantit pas le succès de votre produit, elle vous assure que la manière de l'utiliser ne sera pas un obstacle à son adoption.

Qu'il soit objet, service ou site web, votre produit ne peut plus être immuable ; il gagnera à évoluer perpétuellement afin de répondre aux usages mouvants de vos publics. 


\section{MÉMENTO par Cécile Touitou}

Cette dernière partie permet un rappel des différentes phases à mettre en œuvre pour engager une démarche d'évaluation de l'impact de sa bibliothèque en reprenant les points forts évoqués par les différents contributeurs de l'ouvrage.

Avant de se lancer dans l'évaluation, il convient d'exprimer clairement ses missions, que l'on devra décliner en objectifs stratégiques, et éventuellement en objectifs opérationnels. Effectivement, il n'est plus possible de concevoir une offre de services sans avoir réfléchi au préalable à ses missions ; il n'est plus possible d'énoncer des missions sans avoir interrogé ses usagers sur leurs besoins et leurs attentes. Cette double interrogation devra se faire nécessairement en inscrivant la bibliothèque et son avenir dans le contexte d'un monde en mouvement où les solutions alternatives pour trouver de l'information et se nourrir de nouveaux contenus sont multiples. Quel est alors le rôle de la bibliothèque ? C'est toute la question " existentielle » qu'il convient de se poser au départ de toute démarche d'évaluation. Rien moins que ça!

Cette expression ne pourra se faire qu'en fonction de la connaissance du territoire à desservir et des publics qui le composent. Qu'est-ce qu'on veut faire ? Pour qui ? Pour quoi ? Avant d'arriver à des réponses claires, il convient la plupart du temps de mener à bien les différentes étapes décrites ci-dessous. 
Connaître les publics actuels et potentiels de son établissement permet de décliner une offre de services segmentée adaptée au territoire. Pour cela, en bibliothèque territoriale, on tirera profit de l'exploitation des données mises à disposition par l'Insee. Selon ses besoins, on pourra exploiter les données de la commune ou - plus en détail - les données infracommunales.

Dans le monde des bibliothèques universitaires, il sera important de disposer des chiffres de la scolarité permettant de distinguer les effectifs par année d'étude et par discipline. Ces données à jour de l'année universitaire en cours permettront de mesurer précisément l'usage que font les différents publics des services qui leur sont proposés, et de mesurer le taux d'utilisation des collections imprimées et électroniques qui leur sont destinées. Pour les BU, en complément, on pourra étudier les rapports réalisés par l'Observatoire national de la vie étudiante $(\mathrm{OVE})^{1}$ au niveau national, et, sur le plan local, se rapprocher des antennes régionales de l'Observatoire qui pourront fournir des données fort utiles sur la vie étudiante dans la région où est implantée l'université.

\section{CHOISIR LES « BONS » INDICATEURS}

Ayant identifié son public et ses besoins, fixé ses objectifs stratégiques, la bibliothèque est en mesure de choisir les « bons » indicateurs de suivi. Ils seront « bons " s'ils sont adaptés à sa propre situation et lui permettent d'obtenir une bonne visibilité sur la performance de son activité et la satisfaction de ses usagers.

On pourra choisir les indicateurs pertinents parmi ceux proposés par les normes relatives aux bibliothèques. Certains décriront l'activité de la bibliothèque : les intrants (les ressources mises à disposition de la bibliothèque pour son fonctionnement : collections, personnel, budget, sites web ou les ressources et collections numériques) et les extrants (les résultats de l'activité, par exemple l'usage des collections, la fréquentation des

1. < http://www.ove-national.education.fr/ >. 
animations, l'accès du public aux ordinateurs de la bibliothèque, ou, du côté des ressources numériques, le nombre de pages vues, etc.). D’autres encore décriront des process (délais de catalogage des documents, ou de mise à disposition ; taux de disponibilité du site ; proportion des titres recherchés disponibles dans la collection, etc.). Ces indicateurs d'activité et/ou de performance pourront être utilement complétés, on le verra, par des indicateurs d'impacts.

EXPLOITER LES CHIFFRES DISPONIBLES (LES DONNÉES INDUITES)

Avant de se lancer dans des ratios complexes, il ne faut pas négliger la fréquentation comme première mesure d'impact. Elle fait partie de ce que la norme ISO 16439 appelle les « données induites » décrivant par là tous les chiffres qui sont générés automatiquement (induits) par les systèmes de gestion de bibliothèque ou les compteurs d'entrées dans les bâtiments. Exploiter systématiquement ces chiffres, qui ont le mérite d'être simples à collecter et fiables dans le temps (car toujours mesurés de façon identique), est un premier exercice incontournable qui, par ailleurs, sera un bon indice de la satisfaction des usagers : la fréquentation monte ? N'est-ce pas tout simplement parce que la bibliothèque a bonne réputation et attire de plus en plus de monde ? (Attention cependant aux flux générés par les bibliothèques saturées où les usagers errent à la recherche de places, souvent en BU, ce qui génère des valeurs élevées qui ne sont pas cependant synonymes de satisfaction élevée !).

S'INSPIRER DES RECOMMANDATIONS DES NORMES EN BIBLIOTHÈQUE

Les indicateurs de performance tels que décrits dans la norme IS0 11620:2008 (fr) permettent d'éclairer les processus de décision « au regard des missions, des objectifs généraux et des objectifs opérationnels de la bibliothèque considérée » dans la mesure où ils facilitent l'évaluation de « la qualité et l'efficacité des services et autres activités de la bibliothèque de même que l'efficience avec laquelle la bibliothèque tire parti de ses ressources ». 
Il convient donc que ces indicateurs répondent à un certain nombre de règles que rappelle la norme : un " indicateur doit servir à quelque chose, il doit répondre à un objectif et fournir une aide à la prise de décision » ; il doit répondre à un critère de fiabilité : " un indicateur doit toujours mesurer la même chose lorsqu'il est utilisé à plusieurs reprises, la mesure doit rester la même d'une fois à l'autre »; de validité : « un indicateur doit mesurer ce qu'il est censé mesurer » et de comparabilité* : "dans le cas où l'indicateur est destiné à la comparaison entre plusieurs bibliothèques, il convient également de s'assurer que l'indicateur permet d'obtenir le même résultat pour des niveaux de services égaux ».

SE COMPARER DANS LE TEMPS : LA NOTION DE DIACHRONIE

Cette notion de comparabilité est essentielle dans l'évaluation. On conserve des indicateurs identiques pour regarder comment ils évoluent dans le temps. L'activité est-elle comparable à celle que l'on a observée l'année précédente ? Quelle est la tendance sur plusieurs années ? Y a-t-il des évolutions cohérentes ? Incohérentes ? Que cela nous dit-il sur notre activité, notre gestion, notre impact ? Comment ces données évoluent-elles par rapport au nombre d'usagers à desservir ? L'observation critique de ces évolutions est indispensable à la bonne gestion et à la bonne allocation des ressources (humaines et financières) au sein des différents services de la bibliothèque.

SE COMPARER AUX AUTRES : DE L'USAGE DU BENCHMARK

Pour les bibliothèques municipales, on devra, en complément à l'analyse des données de population, vérifier les données disponibles sur le site de l'Observatoire de la lecture publique ${ }^{2}$ qui met à disposition du grand public et des professionnels un site gratuit de cartographie interactive ${ }^{3}$. Plus

2. < http://www.culturecommunication.gouv.fr/Politiques-ministerielles/Livre-et-Lecture/Bibliotheques/Observatoire-de-la-lecture-publique $>$.

3. < http://www.culturecommunication.gouv.fr/Politiques-ministerielles/Livre-et-Lecture/Bibliotheques/Observatoire-de-la-lecture-publique/cartographie $>$. 
de 200 indicateurs cartographiés peuvent être téléchargés sous forme de tableurs en vue d'une exploitation statistique par l'utilisateur. Le logiciel permet également de générer automatiquement un rapport présentant sous forme de graphiques les chiffres-clés des bibliothèques, pour un territoire sélectionné.

Il faut veiller à ne se comparer qu'à des établissements comparables ! Pour ce faire, l'Observatoire de la lecture publique publie également une synthèse annuelle sur les données d'activité des bibliothèques, qui permet de consulter des "profils moyens » présentant, pour une population donnée, la valeur moyenne des principaux indicateurs, calculée sur une tranche démographique fine (1 500 à 2500 habitants ; 2500 à 4000 habitants, etc. jusqu'à de 150000 à 250000 habitants). En BU, on pourra tirer profit des données collectées par l'Association of Research Libraries $(\mathrm{ARL})^{4}$ via son enquête Libqual+ qui fournit chaque année la mesure des attentes et de la satisfaction des usagers sur une trentaine d'indicateurs relevant de la relation aux services, aux espaces et aux collections. Ces données permettent la comparaison avec des bibliothèques sur le territoire national ou international.

COLLECTER LA SATISFACTION EN CONNAISSANT LE NIVEAU DES ATTENTES

$\checkmark$

Une fois les chiffres « induits » collectés et analysés, on pourra souhaiter en savoir plus sur les attentes et la satisfaction des usagers. De nombreux moyens permettent d'évaluer la satisfaction. Le courrier, les boîtes à idées, les conversations à la banque de prêt sont autant de moyens de capter la perception qu'ont les usagers des services. S’il est possible, on mettra en œuvre un outil de mesure périodique de la satisfaction via un questionnaire permettant de vérifier la perception des usagers et son évolution. Les BU disposent pour le faire de l'outil Libqual+. En BM, il n'existe pas encore d'outil normalisé permettant d'interroger les usagers. À défaut, on pourra consulter utilement la version anglaise de la norme ISO 16439 (Méthodes et procédures pour évaluer l'impact des bibliothèques) dont

4. < http://www.arl.org/ >. 
un livre blanc est paru en français ${ }^{5}$. La norme fournit de nombreuses recommandations permettant la mesure de la satisfaction. Il convient de préciser clairement si on interroge la visite la plus récente (ce qu'il faut privilégier) ou bien si on se rapporte à une appréciation générale de la bibliothèque et de ses services, (ce qui risque de générer des réponses moins pertinentes, met en garde la norme). Un haut niveau de satisfaction des services évalués peut permettre d'identifier ceux qui ont eu un impact positif sur les compétences, les qualifications ou les pratiques de l'usager.

\section{MESURER L'IMPACT AUPRÈS DE L'ENSEMBLE DE LA POPULATION}

Interroger la satisfaction constitue la première étape de la mesure d'impact. On pourra ambitionner de se lancer dans une approche plus globale visant à interroger les usagers et les non-usagers sur l'ensemble des impacts de la bibliothèque. On cherchera à savoir en quoi la bibliothèque a " changé » la vie des usagers, mais aussi des non-usagers, du quartier, ou de la " communauté », comme disent les Anglo-Saxons, voire de la société. L'idée qui sous-tend ces évaluations est que « les bibliothèques changent la vie » pour reprendre le beau slogan de la campagne 2016 de l'American Library Association ${ }^{6}$.

En général, une étude d'impact portera sur les usagers actuels de la bibliothèque afin de capter leur opinion et leur expérience, mais si le point principal de l'enquête est d'interroger la valeur même de la bibliothèque, les non-utilisateurs devront également être interrogés dans la mesure où les personnes qui ne fréquentent pas les bibliothèques peuvent avoir une idée claire de ce qui fait (ou non) leur valeur pour les citoyens et la société en général. Interroger un échantillon de tous les utilisateurs potentiels aidera également à identifier les raisons de la non-utilisation.

Différentes techniques de questionnement des utilisateurs et non-utilisateurs sont présentées dans la norme ISO 16439 qui permet de collecter ce qu'elle désigne par «données sollicitées ». On pourra interroger le

5. Qu'est-ce qui fait la valeur des bibliothèques ? [En ligne] : < http://portailgroupe.afnor.fr/public_ espacenormalisation/AFNORCN46-8/Livre\%20Blanc\%20fev2016.pdf >.

6. Voir le site dédié à cette campagne $:<$ http://www.ilovelibraries.org/librariestransform/ > 
panel au sujet de son expérience de la bibliothèque et sur son estimation des bénéfices que présente la bibliothèque. Les méthodes concernées regroupent le questionnaire d'enquête, l'interrogation orale (interviews, groupes de discussion), l'enregistrement des utilisateurs, etc.

Dans le cadre de formations en BU ou en BM, on pourra soumettre aux participants des questionnaires d'auto-évaluation des compétences avant/ après permettant de mesurer les bénéfices et les compétences acquises. Enfin, des méthodes de mesure d'impact économique peuvent également être utilisées qui permettent de calculer le retour sur investissement.

CROISER LES DONNÉES INDUITES, SOLLICITÉES, OBSERVÉES

$\bar{\downarrow}$

Finalement, la bibliothèque a à sa disposition toute une gamme d'outils et de méthodes pour mesurer son activité, sa performance, la satisfaction des usagers et son impact social et économique. Il est recommandé de croiser les approches : on verra par exemple si les déclarations collectées par questionnaire sur un élargissement des horaires sont corroborées par un usage avéré et mesuré des lieux en soirée... On analysera si un faible niveau d'attente exprimé sur l'offre de formation en BU est accompagné par un haut niveau en matière de compétence informationnelle... En plus de multiplier les angles d'analyses quantitatives (données induites), qualitatives (données déclaratives, sollicitées ou observées), il faudra renouveler périodiquement les enquêtes afin d'observer leur évolution.

REVENIR À « LA GRANDE IMAGE » : MESURER L'IMPACT

EN FONCTION DES OUTCOMES, CONSTRUIRE UN PLAIDOYER

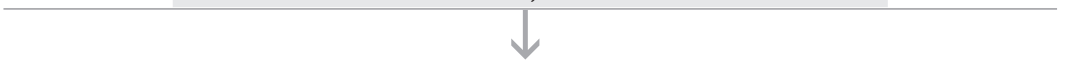

En conclusion, il est important de ne pas perdre de vue la " grande image » et ne pas se noyer dans les détails. On le sait par expérience, une fois qu'on a commencé à mesurer, on a tendance à vouloir tout compter sans que cela ne soit toujours ni utile, ni pertinent. Avant d'évaluer, il faut bien être certain de ce que l'on cherche à observer et ne pas perdre de vue ce que l'on fera des résultats... Interroger sur des actions que l'on n'aura pas les moyens d'amender est peine perdue. 
On l'a dit en en préambule, il convient d'évaluer avec un soin particulier les résultats en rapport avec les missions de l'établissement. C'est ce qui est souvent désigné par la notion d'« outcome » qui décrit une catégorie particulière d'impacts générés par les bibliothèques. Ils sont étroitement liés à ses programmes et missions propres plutôt qu'à l'offre générale des services comme le prêt de livres et la mise à disposition d'une salle pour la consultation de la presse.

Il est important de bâtir avec les usagers une offre de services (et donc son évaluation) selon la valeur et l'intérêt qu'ils lui trouvent : une offre utile et nécessaire, toujours remise en question ; et non pas une offre héritée d'un usage ancien, devenu par le fait même obsolète.

Savoir à quoi on sert, ce qui constitue notre "valeur », comment l'améliorer en relation avec les attentes exprimées par les usagers et les habitants du quartier que l'on dessert (ou la communauté universitaire), distribuer les forces et les ressources là où on est attendu, rétroagir, s'adapter, être " agile » et à l'écoute dans une dynamique d'amélioration continue en fonction des retours des utilisateurs... voilà en quelques mots comment on pourrait décrire une démarche d'évaluation vertueuse.

Menée à bien, cette évaluation qui s'inspire de ce que les usagers veulent et attendent, donne aux bibliothécaires tous les éléments nécessaires pour construire un plaidoyer, négocier des financements, voire l'existence de la bibliothèque et le développement de son action! 


\section{GLOSSAIRE}

\section{Comparabilité}

La norme AFNOR 11620 introduit la comparabilité comme étant un des critères que doivent respecter les indicateurs de performance (au même titre que leur contenu informatif, leur fiabilité, leur validité, etc.). Il est noté qu' « Un indicateur de performance de bibliothèque permet de comparer des bibliothèques entre elles si le même résultat, dans sa précision, signifie le même niveau de qualité de services ou le même niveau d'efficience dans les bibliothèques soumises à comparaison » (§4.2.2).

Un chapitre entier de cette même norme (§5.3.5) s'intitule " Comparabilité des données des indicateurs de performance » où il est précisé que la comparabilité s'entend d'abord dans la possibilité pour un établissement de comparer ses résultats d'une année sur l'autre dans un processus d'autoévaluation. Dans un deuxième temps, la bibliothèque devra pouvoir comparer ses résultats avec ceux d'établissements comparables (en termes de missions, de ressources, de procédures de gestion, etc.).

\section{Contingent Valuation Method (CVM)}

Voir Évaluation contingente

\section{Counter (pour Comptage de}

l'utilisation en ligne des ressources électroniques en réseau)

« Lancé en mars 2002, Counter est une initiative internationale en direction des bibliothécaires, éditeurs et intermédiaires qui propose des normes facilitant l'enregistrement et l'extraction des statistiques des éditeurs de ressources électroniques de manière cohérente, crédible et compatible. Ces normes sont réparties en deux Codes de bonne pratique, l'un portant sur les revues électroniques et les bases de données, et l'autre sur les e-books et les ouvrages de référence. Ce guide est fait pour accompagner pas à pas les fournisseurs tout au long du processus d'agrément ». Source : Couperin.org.

\section{Démarche UX}

Démarche orientée usager, UX désignant User Experience. L'expérience utilisateur correspond, d'après la norme ISO 9241-210, « aux réponses et aux perceptions d'une personne qui résultent de l'usage ou de l'anticipation de l'usage d'un produit, d'un service ou d'un système ». " L'expérience utilisateur » a d'abord été développée pour rendre les logiciels plus utiles, navigables ou accessibles... On peut ainsi parler de l'" utilisabilité » des sites ou des outils numériques. 
L'approche UX tend à se répandre à l'ensemble du design, que ce soit celui de la conception des espaces, des bâtiments, des services... Elle repose toujours sur l'observation et l'interrogation des usagers sur l'usage qu'ils font des services offerts (ou des espaces) et leur appropriation afin de les rendre plus navigables ou praticables; en un mot : utiles !

En bibliothèque, tout un courant se développe autour des travaux d'Andy Priestner qui a organisé en 2015 la première conférence UXLibs consacrée à l'approche UX en bibliothèque.

\section{Donnée induite}

La norme ISO 16439 introduit la distinction entre les données induites qui sont générées automatiquement par les outils informatiques qu'utilise la bibliothèque (compteurs d'entrées, nombre de prêts/retours, etc.), les données sollicitées et observées. Elle recommande de collecter ces trois sources de données, distinctes dans leur nature, pour mesurer l'impact de la bibliothèque.

\section{Donnée observée}

La norme ISO 16439 introduit cette notion pour évoquer les mesures d'impacts. Il s'agit des données que l'on obtient par observation des usagers dans les murs de la bibliothèque pour mieux comprendre leur appropriation des espaces et leur usage des services. Les données des enquêtes du type "Sweeping the library" en sont un exemple. (Voir « donnée induite »).

\section{Donnée sollicitée}

La norme ISO 16439 introduit cette notion pour évoquer les mesures d'impacts. Il s'agit des données que l'on obtient par sollicitation des usagers au travers d'entretiens ou d'enquêtes. (Voir « donnée induite »).

\section{Expérience utilisateur Voir Démarche UX}

\section{Évaluation contingente}

\section{ou Contingent Valuation Method (CVM) en anglais}

Il s'agit d'une méthode d'évaluation économique visant à déterminer par le biais de questionnaires auprès des usagers la valeur attribuée à certains biens ou ressources hors marché, comme les bibliothèques, par exemple. L'usage de ces services ou biens fournit une utilité, mais il est impossible de leur assigner un prix, et, partant, une valeur de marché. On s'appuie alors sur l'évaluation du " consentement à payer » ou du « consentement à renoncer » et du « coût marginal de remplacement » pour fixer ces valeurs. 


\section{Impact de la bibliothèque}

La notion d'impact des bibliothèques a été largement diffusée suite à la parution de la norme ISO 2709. À la mesure de l'activité et de la performance, s'est ajoutée la mesure de l'impact qui vise à évaluer en quoi la bibliothèque change la vie des usagers, des quartiers ou de la société en permettant en accès au savoir et à la culture, un apprentissage du numérique, etc. L'impact peut être sociétal mais économique également (création de valeur, d'emploi, etc.). La bibliothèque par son existence-même et son prestige éventuel est également un lieu structurant sur le territoire qui participe au rayonnement du quartier. L'impact regroupe l'ensemble de ces effets directs et indirects, immédiats ou différés, de la bibliothèque sur son environnement.

\section{Indicateur}

La norme 11620 le définit comme étant une "expression (numérique, symbolique ou verbale) permettant de caractériser des activités (manifestations, objets ou personnes) en termes quantitatifs et qualitatifs afin d'en déterminer la valeur, ainsi que la méthode associée ». L'indicateur permet de mesurer l'efficacité d'une politique pour en apprécier la pertinence et, éventuellement, rétroagir sur les modalités de l'action. Il ne faut pas confondre statistique et indicateur. L'indicateur est le plus souvent une construction regroupant des données qui rapprochent une variable et un ob- jectif (par exemple nombre d'inscrits et population à desservir) alors que la statistique est une donnée brute. Il existe une vaste typologie d'indicateurs (d'efficience, de pertinence, d'efficacité, d'impact, financiers, de performance, de qualité, etc.).

\section{Méthodes agiles}

On désigne par là des pratiques de pilotage et de réalisation de projets initialement utilisées pour la conception de logiciels informatiques. Cependant, depuis quelques années leur champ d'application s'élargit et touche maintenant également le monde des bibliothèques. Le principe fondateur de ces méthodes repose sur un cycle de développement itératif, incrémental et adaptatif. La contribution de Nathalie Clot illustre bien le déploiement de ces méthodes en bibliothèque où pour avancer, il conviendrait de "Arrêter, commencer, continuer » afin d'introduire un processus d'amélioration continue de la qualité. L'adoption d'un mode adaptatif de pilotage de projet permet à la bibliothèque de modifier ses besoins en cours de projet en fonction du retour qu'il aura de ses usagers.

\section{Outcome}

La notion d'« outcome » en bibliothèque pourrait être traduite en français par résultats ou impacts dans la mesure où ces résultats seraient liés aux missions de l'établissement. La norme ISO 16439 introduit cette notion 
en complément des résultats d'activité ou de performance qui caractérisent habituellement les évaluations en bibliothèque. Le livre blanc de la commission Afnor CN46-8 précise que : "Pour certains auteurs, l'« outcome " désigne l'ensemble des transformations ou des changements induits dans la vie, l'expérience ou la pratique de l'usager ou de la communauté en relation avec les missions d'éducation et de mise à disposition de la documentation des bibliothèques. »

La Public Library Association a conçu tout un projet appelé " Outcome » lancé par sa présidente Carolyn Anthony en août 2013 où elle déclare dans un article au titre prometteur (New Measures for a New Era) : «Ce qui est nécessaire est un éventail de mesures de la performance qui peuvent capter l'ensemble des services que les bibliothèques publiques rendent à leurs communautés comprenant des lignes directrices qui permettent d'en assurer la cohérence et la validité. Nous avons également besoin de mesurer les résultats (en anglais Outcome) ou l'impact des transformations que certains de ces services opèrent dans la vie des individus et le bien-être de la communauté » (Voir Public Libraries Online, juillet/août 2013).

\section{Satisfaction des usagers}

La norme 11620 décrit la satisfaction des usagers comme un indicateur permettant d'évaluer la mesure dans laquelle les usagers sont satisfaits des services de la bibliothèque considérés dans leur totalité ou bien isolément. On peut ainsi interroger sur un ou plusieurs services et notamment : les horaires d'ouverture ; les installations pour l'étude ; la disponibilité des documents ; le service de prêt entre bibliothèques ; le service de renseignement et de référence ; etc. Le suivi de la satisfaction dans le temps pour en scruter les variations est recommandé. Les résultats de ce suivi peuvent alimenter un tableau de bord. Il y a plusieurs méthodes pour mesurer la satisfaction : les enquêtes, les entretiens, et, de façon indirecte, l'usage des services. On pourra souhaiter contextualiser la mesure de la satisfaction en interrogeant les usagers à la fois sur leurs attentes minimales, sur leurs souhaits et sur la satisfaction du service utilisé (comme le fait l'enquête Libqual + selon la méthode Servqual, outil marketing permettant de mesurer l'écart perception-attentes de la qualité globale du service fourni).

\section{Smart City \\ Voir « Ville intelligente »}

\section{Valeur d'usage}

Valeur qualitative (et subjective) attribuée par un individu aux avantages économiques de l'utilisation d'un service ou d'un bien en fonction de la satisfaction qu'il lui procure. 
Valeur de non-usage

On peut ne pas avoir usage d'un bien ou d'un service et pourtant en tirer profit d'une certaine façon, immédiatement ou dans le futur, pour soi, ou pour les individus autour de soi. Les valeurs de non-usage regroupent la valeur d'option (pour un usage futur), la valeur d'existence, et la valeur de transmission.

«Ville intelligente "

\section{ou Smart City en anglais}

On décrit ainsi une ville utilisant les technologies de l'information pour développer la qualité et les performances des services urbains. Dans ce contexte, la bibliothèque peut tirer parti de l'ouverture des données administratives pour mieux répondre aux besoins des usagers, et, inversement ouvrir les données d'usage de la bibliothèque et permettre au citoyen de s'en saisir pour de multiples exploitations.

\section{Zone de chalandise}

\section{ou zone d'attraction ou zone}

\section{de rayonnement de la bibliothèque}

Cette notion largement présentée dans la contribution d'Emmanuelle Asselin est empruntée au marketing. Elle décrit la zone géographique d'influence de la bibliothèque : la zone de provenance des usagers de cet établissement. 


\section{INDEX}

A

Autoévaluer 106

B

Bénéfice commercial 31

Bénéfice social 31

C

Comparabilité 162

Consentement à accepter 32

Consentement à payer 31-32

Consentement à renoncer 32

Counter 18, 77, 141

Coût d'opportunité 30-31

Coût marginal de remplacement 32

Coût moyen de déplacement 32

D

Démarche UX 12, 76, 85. Voir Expérience utilisateur

Donnée bibliométrique 120

Donnée induite 13, 25, 73, 129, 136

Donnée observée 13, 26, 74, 129-130, 136
Donnée sollicitée 13, 26, 74, 129-130, 136

E

Enquête d'observation 127, 130. Voir Sweeping

Évaluation contingente 31

Expérience utilisateur 85, 132, 151. Voir Démarche UX

Externalité 29

ezPAARSE 18, 140-146, 148-149

$\mathrm{F}$

Fiche diagnostic 94

Fiche partenaire 94

I

Impact de la bibliothèque $14,19,25$, 27-28, 45, 52, 88, 108

Indicateur 9, 12, 17, 19, 24, 40-41, 43-45, 47-52, 58-59, 61, 75-83, 85, 87, 90-92, 95-96, 109, 149

Itération 139 
L

Libération des données 42, 44-45,

49,58

M

Méthode agile 141

0

Open data 42-44, 58

Outcome 12, 65, 132

$\mathbf{P}$

Performance clef 90

Politique documentaire 129, 135, 140, 148

$\mathbf{R}$

Rapport d'activité annuel 49

READ (échelle ou méthode) 110-116, 118

S

Satisfaction des usagers 11, 12, 14, 25, 119, 163

Sweeping 127. Voir enquête d'observation
$\mathrm{T}$

Test blanc 153

Tri par cartes 137, 139

Troisième lieu 31

$\mathrm{V}$

Valeur de non-usage 29

Valeur d'existence 33. Voir valeur de non-usage

Valeur d'héritage. Voir valeur de nonusage

Valeur d'option. Voir valeur de nonusage

Valeur de transmission. Voir valeur de non-usage

Valeur d'usage 29

Ville intelligente 41

\section{Z}

Zone d'attraction. Voir zone de chalandise

Zone de chalandise $12,52-55,58-59$, 61, 64, 94

Zone de rayonnement de la bibliothèque. Voir zone de chalandise 


\section{BIBLIOGRAPHIE}

Afnor, Indicateurs de performance des bibliothèques : information et documentation: norme internationale ISO 11620 , Paris, Afnor, 2008.

Afnor, Statistiques internationales des bibliothèques : information et documentation: norme internationale ISO 2789, Paris, Afnor, 2008.

Afnor CN 46-8, Qualité-Statistiques et évaluation des résultats. Qu'est-ce qui fait la valeur des bibliothèques?, février 2016. [En ligne] : < http://portailgroupe.afnor.fr/public_espacenormalisation/AFNORCN46-8/Livre\%20 Blanc\%20fev2016.pdf >.

Alonzo Valérie, Renard Pierre-Yves (dir.), Evvaluer la bibliothèque, Paris, Éditions du Cercle de la Librairie, 2012 (coll. Bibliothèques).

Altman Ellen, Hernon Peter et Dugan

Robert E., Assessing Service Quality: Satisfying the Expectations of Library Customers, Chicago, ALA Editions, 2015.

Amar Muriel et Béguet Bruno, « Les semaines tests : évaluation de l'utilisation des collections imprimées sur place à la Bpi ", Bulletin des bibliothèques de France, 2006, n 6, pp. 3642. [En ligne] : < http://bbf.enssib.fr/ consulter/bbf-2006-06-0036-007 >.
Benhamou Françoise et Chantepie Philippe, «Culture et économie : chiffres et cryptes », Bulletin des bibliothèques de France, 2016, $n^{\circ} 8$, pp. 8-18. [En ligne] : < http://bbf.enssib. fr/consulter/bbf-2016-08-0008-001 >.

Calenge Bertrand, "Quels tableaux de bord ? "Bulletin des bibliothèques de France, 2008, $\mathrm{n}^{\circ} 3$, pp. 35-38. [En ligne] : < http://bbf.enssib.fr/consulter/ bbf-2008-03-0035-004 >.

Carbone Pierre (dir.), Construire des indicateurs et tableaux de bord, Paris, Éditions Tec \& Doc ; Villeurbanne, Presses de l'enssib, 2002 (coll. La Boîte à outils ; 15).

Evans Christophe (dir.), Mener l'enquête. Guide des études de publics en bibliothèque, Villeurbanne, Presses de l'enssib, 2011 (coll. La Boîte à outils ; 22).

Giappiconi Thierry (dir.), Manuel théorique et pratique d'évaluation des bibliothèques et centres documentaires, Paris, Éditions du Cercle de la Librairie, 2001 (coll. Bibliothèques).

Hernon Peter, Altman Ellen et Dugan Robert E., Assessing Service Quality: Satisfying the expectations of library customers, $3^{\text {rd }}$ edition, Londres, Facet Publishing, 2015. 
Huysmans Frank et Oomes Marjolein, "Measuring the public library's societal value: A methodological research program", IFLA Journal, 2013, 39 (2), pp. 168-177.

ISO 16439 : 2014 (en), Information and documentation: Methods and procedures for assessing the impact of libraries. [En ligne] : < https://www. iso.org/obp/ui/\#iso:std:iso:16439:ed1:v1:en >.

Jouneau Thomas et Porquet Thomas, « Exploiter les traces de passage vers la documentation numérique ", Journée d'étude : Exploiter les données d'usages en bibliothèque : pour quoi faire ?, 14 janvier 2016. [En ligne] : < http://www.enssib.fr/JEExploiter-les-donnees-d-usages-enbibliotheque $>$.

Kingma Bruce et McClure Kathleen, "Lib-Value: Values, Outcomes, and Return on Investment of Academic Libraries, Phase III: ROI of the Syracuse University Library", College \& Research Libraries, January 2015, 76 (1). [En ligne] : < http://crl.acrl.org/ content/early/2014/01/17/crl13-485. full.pdf + html $>$.

Kyrillidou Martha, "From Input and Output Measures to Quality and Outcome Measures, or, from the User in the Life of the Library to the Library in the Life of the User", Journal of Academic Librarianship, 2002, 28, $\mathrm{n}^{\circ} 1-2$, pp. 42-46.
Lyons Ray, Curry Lance Keith, "Outputs, outcomes, \& other data", Library Journal, 3 novembre 2014. [En ligne] : < http://lj.libraryjournal.com/2014/11/ managing-libraries/lj-index/classof-2014/the-star-libraries-2014 >.

Nitecki Danuta A., Dugan Robert E. et Hernon Peter (collab.), Viewing Library Metrics from Different Perspectives: Inputs, Outputs, and Outcomes, Santa Barbara, ABC-CLIO, 2009.

Oomes Marjolein, Public library impact of individuals and society: A literature review (working title), Unpublished manuscript, Den Haag, Koninklijke Bibliotheek, forthcoming.

Papin Dominique, Impact des bibliothèques sur la réussite des étudiants : survol de la littérature récente. Version révisée du 14 janvier 2016. [En ligne] : < https://tribuneci.files.wordpress. com/2016/01/impact-biblio-reussite_2016-01-14.pdf >.

Poll Roswitha, "Can we quantify the library's influence? Creating an ISO standard for impact assessment", Performance Measurement and Metrics, 2012, 13 (2), pp. 121-130.

Renard Pierre-Yves, "ISO 2789 and ISO 11620: Short presentation of standards as reference documents in an assessment process", LIBER Quarterly, 2007, 17 (3/4).

Renard Pierre-Yves, « La normalisation des statistiques et des indica- 
teurs : de l'inventaire à l'évaluation ", Bulletin des bibliothèques de France, 2008, n 3, pp. 29-34. [En ligne] :

$<$ http://bbf.enssib.fr/consulter/bbf2008-03-0029-003 >.

Vakkari Pertti, Svanhild Aabø,

Ragnar Audunson, Huysmans Frank and Oomes Marjolein, "Perceived outcomes of public libraries in Finland, Norway and the Netherlands", Journal of Documentation, 2014, 70 (5), 927-944.
Vakkari Pertti, Svanhild Aabø, Ragnar Audunson, Huysmans Frank, Kwon Nahyun, Oomes Marjolein and Sei-Ching Joanna Sin, "Patterns of perceived public library outcomes in five countries", Journal of Documentation, 2016, 72 (2), 342-361. 


\section{LISTE DES ILLUSTRATIONS}

Tableau. Indicateurs et statistiques : trois rapports techniques

p. 22

Tableau. Trois normes phares complémentaires

p. 23

Tableau. Valeur du site web de la British Library

p. 34

Tableau. Calcul du ratio coûts-avantages

pour la British Library, 2011-2012, M£

p. 35

Tableau. Apport des BU aux performances individuelles

et institutionnelles en matière de recherche

p. 37

Figure. L'impact de la bibliothèque sur la population en mars .............. p. 46

Tableau. Évaluer la zone de chalandise de la bibliothèque :

indicateurs à retenir

p. 60

Tableau. Évaluer la zone de chalandise de la bibliothèque : exploitation des indicateurs.

Tableau. Comparaison des extrants et outcomes des bibliothèques publiques

Encadré. Six types d'outcomes pour les bibliothèques publiques p. 68

Schéma. La valeur des bibliothèques publiques appréhendée à travers cinq dimensions

Tableau. L'impact du changement des règles d'emprunt :

nombre de prêts vs durée de circulation.

Tableau. Évolution entre mai 2015 et mai 2016 des indicateurs clés de Google Analytics (avant/après simplification drastique du site) p. 84

Encadré. Trois fiches diagnostic. p. 92 
Encadré. Mesurer la fréquentation : les cinq étapes de la mise en œuvre p. 97

Formulaire de codage. Grille d'analyse des interactions. p. 113

Tableau. Exemple de formulaire saisi par les bibliothécaires pendant la période-test d'analyse des interactions à la Bpi p. 115

Diagramme. Représentation en radars des résultats de LibQUAL ........p. 124 Encadré. Recommandations de la norme ISO 16439 p. 129

Graphique. Répartition des trois motifs principaux de visite à la bibliothèque. Pourcentages calculés sur la base des réponses pour chaque rang (1, 2 et 3$)$ p. 133

Encadré. Les trois temps du tri par cartes p. 137

Encadré. Présentation d'une ligne de log standard p. 142

Encadré. Améliorer l'utilisabilité du site : quatre bénéfices principaux 


\section{LISTE DES AUTEURS}

\section{Nicolas Alarcon}

Chef de projet Open Access et chargé de mission Enquêtes-Indicateurs, Service commun de la documentation, université de La Réunion (Saint-Denis)

\section{Muriel Amar}

Chargée d'études, Service et études et recherche, Bibliothèque publique d'information (Paris)

\section{Emmanuelle Asselin}

Consultante associée, Tosca consultants (Paris)

\section{Cécile Avallone}

Directrice de la Bibliothèque départementale du Val d'Oise (Pontoise)

\section{Françoise Benhamou}

Économiste, professeur des universités, université Paris 13

\section{Frédéric Brodkom}

Directeur de la Bibliothèque et Learning center des sciences et technologies, chargé de cours invité en bibliothéconomie et bibliométrie, université catholique de Louvain (Belgique)

\section{Nathalie Clot}

Directrice de la Bibliothèque universitaire d'Angers

\section{Hélène Coste}

Responsable du pôle Sciences sociales, chargée de mission Évaluation et Formation des usagers,
Bibliothèque universitaire, université du Havre

\section{Nadine Delcarmine}

Directrice de bibliothèque adjointe, SID de l'université Grenoble AlpesINP

\section{Frank Huysmans}

Professeur en sciences de l'information et des bibliothèques, université d'Amsterdam et consultant à WareKennis, La Haye (Pays-Bas)

\section{Marie-Christine Jacquinet}

Responsable du Pôle Développement culturel, Conseil départemental des Yvelines (Versailles)

\section{Thomas Jouneau}

Bibliothèque numérique, université de Lorraine (Metz)

Marc Maisonneuve

Gérant et consultant, Tosca

consultants (Paris)

\section{Marjolein 0omes}

Conseillère stratégique, Bibliothèque Koninklijke, La Haye (Bibliothèque nationale des Pays-Bas) et doctorante, Département des sciences humaines, université d'Amsterdam (Pays-Bas)

\section{Joris Paillaré}

Chef de projets numériques, Bibliothèque de Sciences Po Paris 


\section{Thomas Porquet}

Chargé de mission pour le département Services et Prospective, Consortium Couperin.org (Paris)

\section{Guilaine Thuillart}

Chef du service Coordination de l'accueil (Département des publics) à la Bibliothèque publique d'information (Paris)

\section{Cécile Touitou}

Responsable de la mission Marketing, Bibliothèque de Sciences Po Paris 


\section{LA BOÎTE À OUTILS}

Fondée par Bertrand Calenge en 1995.

Les manuels de cette collection visent à fournir aux professionnels des ouvrages pratiques pour conduire des projets bibliothéconomiques d'actualité concernant aussi bien des bibliothèques publiques que des bibliothèques universitaires ou de recherche. Écrit à plusieurs mains, chaque volume est coordonné par un professionnel des bibliothèques.

\section{Catherine Jackson}

directrice de la collection
$+++++++++++++++++++++$

\section{PRESSES DE L'enssib}

École nationale supérieure des sciences de l'information et des bibliothèques

17-21 boulevard du 11 novembre 1918 69623 Villeurbanne Cedex

Tél. 0472444343

Fax 0472444344

< http://www.enssib.fr/presses >
Dans la même collection, à paraître

$++++++++++++++++++++++++++++++++++++$

BA0 \#38 (2016)

Mutualiser les pratiques documentaires : bibliothèques en réseau

sous la direction de Jérôme Pouchol

Déjà parus

$++++++++++++++++++++++++++++++++++++$

BA0 \#36 (2016)

Repenser le fonds local et régional en bibliothèque sous la direction de Claire Haquet et Bernard Huchet

BA0 \#35 (2016)

Médiatiser la science en bibliothèque

sous la direction de Justine Ancelin

BA0 \#34 (2015)

Jouer en bibliothèque

sous la direction de Julien Devriendt

BA0 \#33 (2015)

Construire des pratiques participatives

dans les bibliothèques

sous la direction de Raphaëlle Bats

BA0 \#32 (2015)

Conduire le changement

en bibliothèque : vers

des organisations apprenantes

sous la direction de Christophe Pérales

BA0 \#31 (2014)

Ouvrir plus, ouvrir mieux :

un défi pour les bibliothèques

sous la direction de Georges Perrin

BA0 \#30 (2014)

Produire des contenus documentaires en ligne: quelles stratégies pour les bibliothèques?

sous la direction de Christelle di Pietro

Suite des titres parus page suivante 
Déjà parus

$++++++++++++++++++++++++++++++++++++++++++++++++++++++++++$

BA0 \#29 (2014)

Intégrer des ressources numériques dans les collections

sous la direction de Géraldine Barron et Pauline Le Goff-Janton

BA0 \#28 (2013)

Favoriser l'insertion professionnelle

et l'accès à l'emploi : les atouts

des bibliothèques

sous la direction de Georges Perrin

BA0 \#27 (2012)

Faire connaître et valoriser

sa bibliothèque : communiquer

avec les publics

sous la direction de Jean-Marc Vidal

BA0 \#26 (2012)

Apprendre à gérer des collections

patrimoniales en bibliothèque

sous la direction de Dominique Coq

BA0 \#25 (2012)

Développer la médiation documentaire numérique

sous la direction de Xavier Galaup

BA0 \#24 (2011)

Mener un projet international: bibliothèques françaises et coopération internationale

sous la direction de Raphaëlle Bats

BA0 \#23 (2011)

Créer des services innovants. Stratégies et répertoire d'actions pour les bibliothèques sous la direction de Marie-Christine Jacquinet
BA0 \#22 (2011)

Mener l'enquête! Guide des études

de publics en bibliothèque

sous la direction de Christophe Evans

BA0 \#21 (2010)

Communiquer! Les bibliothécaires, les décideurs et les journalistes sous la direction de Jean-Philippe Accart

BA0 \#20 (2010)

Mettre en ouvre un service de questionsréponses en ligne

sous la direction de Claire Nguyen

BA0 \#19 (2010)

Numériser et mettre en ligne sous la direction de Thierry Claerr et Isabelle Westeel

BA0 \#18 (2009)

Mettre en œuvre un plan de classement sous la direction de Bertrand Calenge

BA0 \#17 (2009)

Favoriser la réussite des étudiants sous la direction de Carine El BekriDinoird

BA0 \#16 (2008)

Gérer les périodiques

sous la direction de Géraldine Barron 
Cet ouvrage a été réalisé avec Métopes,

(méthodes et outils pour l'édition structurée

XML-TEI), chaîne éditoriale développée par

le pôle Document numérique de la Maison

de la recherche en sciences humaines de

l'université de Caen.

Secrétariat d'édition:

Silvia Ceccani

Mise en page:

Cédric Vigneault

Conception graphique:

atelier Perluette, 69001 Lyon.

< http://www.perluette-atelier.com >

Achevé d'imprimer en septembre 2016

imprimerie Bialec (Heillecourt) - $\mathrm{n}^{\circ} 89308$

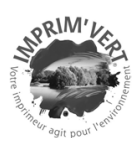

dépôt légal: $2^{\mathrm{e}}$ semestre 2016 